

\title{
Magnetotelluric Data Collected Near Geophysically Logged Boreholes in the Española and Middle Rio Grande Basins, New Mexico
}

By Jackie M. Williams and Brian D. Rodriguez

Open-File Report 2006-1120

U.S. DEPARTMENT OF THE INTERIOR

U.S. GEOLOGICAL SURVEY 


\section{U.S. Department of the Interior \\ Gale A. Norton, Secretary \\ U.S. Geological Survey \\ P. Patrick Leahy, Acting Director}

U.S. Geological Survey, Reston, Virginia 2006

Revised and reprinted: 2006

For product and ordering information:

World Wide Web: http://www.usgs.gov/pubprod

Telephone: 1-888-ASK-USGS

For more information on the USGS - the Federal source for science about the Earth, its natural and living resources, natural hazards, and the environment:

World Wide Web: http://www.usgs.gov

Telephone: 1-888-ASK-USGS

Any use of trade, product, or firm names is for descriptive purposes only and does not imply endorsement by the U.S. Government.

Although this report is in the public domain, permission must be secured from the individual copyright owners to reproduce any copyrighted materials contained within this report. 


\section{Contents}

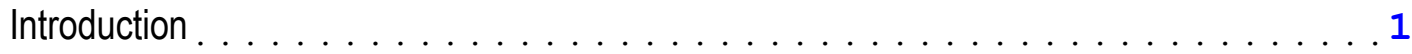

Electrical Rock Properties $\ldots \ldots \ldots \ldots \ldots \ldots \ldots \ldots$



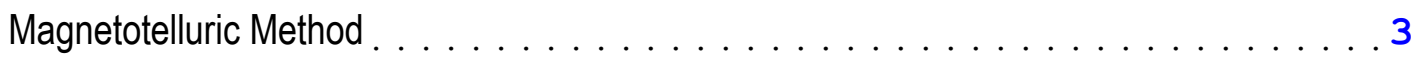

Magnetotelluric Survey . . . . . . . . . . . . . . . . . . . . 4

Magnetotelluric Data . . . . . . . . . . . . . . . . . . . . 4



Appendix 1 Magnetotelluric Data Plots . . . . . . . . . . . . . . . . . 9 


\section{Introduction}

The Santa Fe region is growing rapidly. The Santa Fe Group aquifer in the Española Basin is the main source of municipal water for the region (fig. 1), and water shortfalls could have serious consequences. Future growth and land management in the region depend on accurate assessment and protection of the region's ground-water resources. An important issue in managing the ground-water resources is a better understanding of the hydrogeology of the Tertiary Santa Fe Group. The Santa Fe Group includes the sedimentary deposits that fill the Rio Grande rift and contain the principal ground-water aquifers.

The U.S. Geological Survey (USGS) is conducting a series of multidisciplinary studies of the Española Basin in northern New Mexico. Detailed geologic mapping, high-resolution airborne magnetic surveys, electromagnetic surveys, and hydrologic, lithologic, and hydro-geochemical data are being used to better understand the aquifer systems. Magnetotelluric (MT) surveys were completed as part of these studies (Williams and Rodriguez, 2001; 2003; 2005a; 2005b). The primary purpose of the MT surveys was to map changes in electrical resistivity with depth that are related to differences in various rock types that help control the properties of aquifers in the region.

Resistivity modeling of the MT data can be used to investigate buried structures related to the basic geologic framework of the study area. The purpose of this report is to release MT sounding data collected near geophysically logged boreholes in the study area, including the nearby Middle Rio Grande Basin. This MT data can be used in subsequent resistivity modeling. No interpretation of the data is included in this report.

\section{Electrical Rock Properties}

Electromagnetic geophysical methods detect variations in the electrical properties of rocks - in particular electrical resistivity, or its inverse, electrical conductivity. Electrical resistivity can be correlated with geologic units on the surface and also at depth by using lithologic logs to provide a three-dimensional (3-D) picture of subsurface geology. In the upper crust, the resistivity of geologic units is largely dependent upon their fluid content, pore-volume porosity, interconnected fracture porosity, and conductive mineral content (Keller, 1989). While there is not a one-to-one relationship between lithology and resistivity, there are general correlations that can be made using typical values even though values can be found at other localities that may fall outside of the ranges presented below (Palacky, 1987). Fluids within the pore spaces and fracture openings, especially if saline, can reduce resistivities in what would otherwise be a resistive rock matrix. Resistivity can also be lowered by the presence of electrically conductive clay minerals, graphitic carbon, and metallic mineralization. It is common, for example, for altered volcanic rocks to contain replacement minerals that have resistivities ten times lower than those of the surrounding rocks (Nelson and Anderson, 1992). Saturated, fine-grained sediments, such as clay-rich alluvium, marine shales, and other mudstones, are normally conductive from a few ohm-meters (ohm-m) to a few tens of ohm-m (Keller, 1987; Palacky, 1987). In-situ measurement from local data in Deszcz-Pan and others (2000) show that coarser-grained sediments, such as gravels and clean 




Figure 1. Magnetotelluric (MT) profile index map of northern New Mexico. MT stations acquired in September 1999, July 2002, and July 2004 are numbered solid black boxes. Open circles are nearby geophysically logged boreholes (CWKF1-Castle West Kelly Federal 1, SSF3 - Shell Santa Fe 3, RR9- Rio Rancho 9, SFP1 - Santa Fe Pacific 1, RR18- Rio Rancho 18. Numbered black lines are major highways. Dashed lines are Española and Middle Rio Grande basin boundaries. Map datum is Clark 1866 Spheroid, NAD 1927 CONUS with geographic projection. 
sands, are commonly moderately conductive (tens of ohm-m). Others have reported similar in-situ resistivity measurements for sands and gravels elsewhere in North America (Gilson and others, 2000: Alger, 1971). Metamorphic rocks (non-graphitic) and unaltered, unfractured igneous rocks are normally moderately to highly resistive (a few hundreds to thousands of ohm-m). Carbonate rocks can have similarly high resistivities depending on their fluid content, porosity, and impurities (Keller, 1987; Palacky, 1987). Fault zones may be moderately conductive (tens of ohm-m) when they are comprised of rocks fractured enough to have hosted fluid transport and consequent mineralogical alteration (Eberhart-Phillips and others, 1995). Higher subsurface temperatures cause higher ionic mobility that reduces rock resistivities (Keller, 1987; Palacky, 1987). Tables of electrical resistivity for a variety of rocks, minerals, and geological environments may be found in Keller (1987) and Olhoeft (1989), although these resistivity measurements were laboratory measurements, and using them for in-situ measurements is problematic. Palacky (1987) presents a figure of typical ranges of resistivities of various earth materials, but whether the figure was derived from entirely in-situ measurements is not clear.

\section{Magnetotelluric Method}

The MT method is a passive surface electromagnetic geophysical technique that measures variations in the Earth's natural electromagnetic field to investigate the electrical resistivity structure of the subsurface from depths of tens of meters to tens of kilometers (Vozoff, 1991). Worldwide lightning activity at frequencies of 10,000 to $1 \mathrm{Hertz}(\mathrm{Hz})$ and geomagnetic micro-pulsations at frequencies of 1 to $0.001(\mathrm{~Hz})$ provide the majority of natural signal used by the MT method. The natural electromagnetic wave propagates vertically in the earth due to the large resistivity contrast between the air and the earth causing a vertical refraction of the electromagnetic wave transmitted into the Earth (Vozoff, 1972).

The natural electric and magnetic fields are recorded in two orthogonal, horizontal directions at the Earth's surface (the vertical magnetic field is also recorded). The resulting time-series signals are used to derive tensor apparent resistivities and phases by first converting them to complex cross spectra using Fourier transform techniques. Least squares, cross-spectral analysis (Bendat and Piersol, 1971) are used to solve for a tensor transfer function. Prior to conversion to apparent resistivity and phase, the tensor is normally rotated into principal directions that usually correspond to the direction of maximum and minimum apparent resistivity. For a two-dimensional (2-D) Earth, in which Earth resistivity structure varies with depth and in one lateral direction, the MT fields can be decoupled into transverse-electric (TE) and transverse-magnetic (TM) modes. The 2-D resistivity modeling is generally computed to fit both modes. When the geology satisfies the 2-D assumption, the MT data for the TE mode represents electric fields oriented parallel to geologic strike, and the data for the TM mode represents electric fields oriented perpendicular to strike. The MT method is well suited for studying complicated geological environments because the electric and magnetic fields are sensitive to vertical and horizontal variations in resistivity. The method is capable of establishing whether the electromagnetic fields are responding to subsurface rock bodies of effectively 1, 2, or 3 dimensions. An introduction to the MT method and references 
for a more advanced understanding are contained in Dobrin and Savit (1988) and Vozoff (1991).

\section{Magnetotelluric Survey}

Nine MT soundings were collected in September 1999, July 2002, and July 2004 near geophysically logged boreholes in the study area, including the nearby Middle Rio Grande Basin. Station locations were chosen for proximity to roads and to avoid electrical noise such as power lines. All data at the stations were collected with a portable Electromagnetic Instruments, Inc. (EMI), MT-1 system (EMI, Inc., 1996). Horizontal electric fields were recorded using copper sulfate porous pots placed in an L-shaped, three-electrode array with dipole lengths of 30 meters $(\mathrm{m})$. The orthogonal, horizontal magnetic fields in the direction of the electric-field measurement array were sensed using high magnetic permeability, mu-metal-cored induction coils. Frequencies were sampled from 0.009 to $70 \mathrm{~Hz}$, using single-station recordings of the orthogonal, horizontal components of the electric and magnetic fields and the vertical magnetic field.

The following table lists the 9 MT station locations. Coordinates are referenced to the 1866 Clarke spheroid and North American 1927 Western United States datum. Longitude and latitude format is degrees: minutes: seconds. Universal Transverse Mercator (UTM) units and station elevations are in meters. The accuracy of the $\mathrm{x}, \mathrm{y}$ component is $\pm 5 \mathrm{~m}$. The accuracy of the $\mathrm{z}$ component is $\pm 10 \mathrm{~m}$.

\begin{tabular}{llllll} 
Station & Longitude & Latitude & North(m) & East(m) & Elevation \\
\hline EB01 & $-106: 02: 02$ & $35: 56: 50$ & $3,978,396$ & $13,406,745$ & 1795 \\
EB02 & $-105: 48: 29$ & $35: 59: 23$ & $3,982,900$ & $13,427,161$ & 2509 \\
EB03 & $-105: 55: 03$ & $35: 56: 09$ & $3,977,013$ & $13,417,246$ & 2098 \\
RR01 & $-106: 54: 48$ & $35: 17: 32$ & $3,906,919$ & $13,326,002$ & 1775 \\
RR02 & $-106: 46: 40$ & $35: 20: 50$ & $3,912,809$ & $13,338,440$ & 1829 \\
RR20a & $-106: 37: 05$ & $35: 20: 51$ & $3,912,588$ & $13,352,962$ & 1682 \\
RR21a & $-106: 40: 11$ & $35: 21: 20$ & $3,913,545$ & $13,348,281$ & 1754 \\
RR22a & $-106: 43: 35$ & $35: 20: 52$ & $3,912,787$ & $13,343,119$ & 1780 \\
RR23a & $-106: 50: 09$ & $35: 18: 19$ & $3,908,252$ & $13,333,083$ & 1886 \\
\hline
\end{tabular}

\section{Magnetotelluric Data}

The recorded time-series data were converted to the frequency domain and processed to determine a resistivity and phase tensor at each site. Rotation of the impedance tensor to maximum and minimum directions allowed for decoupling into the TE and TM modes.

Although remote reference techniques were not used in the study, cross-power files were sorted to select optimal signal-to-noise time-series data sets (see Appendix 1).

The effects of near-surface resistivity anomalies can give rise to "static shifts" in the data (Sternberg and others, 1988). Static shifts were significant at stations EB02, EB03, RR2, RR21a, RR23, and RR23a. Cultural features can furthermore affect the response of the MT system. Fences, pipelines, communication lines, power lines, power generators, railways, moving vehicles and trains, and other man-made sources of electromagnetic noise can contaminate the responses. 
The figures in Appendix 1 represent the field-processed MT data for each station after the time-series data were converted to the frequency domain, and after the tensor-transfer function was rotated into principal directions as described in the "Magnetotelluric Method" section of this report.

For each station, nine separate plots are given:

1. Apparent Resistivity ( $\mathrm{x}$ and o symbols are $\mathrm{xy}$ and $\mathrm{yx}$ components, respectively)

2. Impedance Phase ( $\mathrm{x}$ and o symbols are $\mathrm{xy}$ and $\mathrm{yx}$ components, respectively)

3. Rotation Angle

4. Impedance Skew

5. Multiple Coherency ( $\mathrm{x}$ and o symbols are $\mathrm{xy}$ and yx components, respectively)

6. Impedance Polar Plots

7. Tipper Magnitude

8. Tipper Strike

9. HzHx (x symbol) and HzHy (o symbol) Coherency

Error bars (],[) on the Apparent Resistivity, Impedance Phase, Skew, Tipper Magnitude, and Tipper Strike plots represent probable errors within one standard deviation of the sample variance (Gamble and others, 1979).

Apparent resistivity is the ratio of the electric field strength magnitude over the magnetic field strength magnitude for a given frequency. The impedance phase is proportional to the slope of the apparent-resistivity curve on a log-log plot, relative to a baseline at -45 degrees (Vozoff, 1991). A measure of the dimensionality for MT data is provided by the impedance skew of the impedance tensor (Vozoff, 1972). If the effective, measured resistivity response to the geology beneath an MT station is truly one- or two-dimensional, then the skew will be zero. Both instrument and environmental sources of noise contribute to non-zero skew values but are typically small (about 0.1 ) for relatively low-noise-level recordings. Higher skews (more than 0.2 ) indicate either the resistivity response to 3-D geology or higher levels of noise.

In the study area, noise from a number of small power lines and small moving vehicles was negligible at distances of $0.4 \mathrm{~km}$ and greater from the noise source. Power-line amplitude was measured at each site and was typically less than 20 percent of the maximum recordable signals. Noise from larger power lines, power generators, pipelines, and trains was negligible at distances of more than $5 \mathrm{~km}$ from the site. Local lightning, wind, and rainstorms also can degrade data quality, but these were avoided by not recording during active thunderstorm periods. Burying the magnetic induction coils and keeping the electric dipole wires flat on the ground surface helped to minimize wind noise.

Predicted values of the electric field can be computed from the measured values of the magnetic field (Vozoff, 1991). The coherence of the predicted electric field with the measured electric field is a measure of the signal-to-noise ratio provided in the multiple coherency plots. Values are normalized between 0 and 1 , where values at 0.5 signify signal levels equal to noise levels. For this data set, coherencies generally were at an acceptable level, except at times in the frequency "dead band" $(0.01$ to $5 \mathrm{~Hz})$ when they dropped below 0.75 (Dobrin and Savit, 1988).

The figures in Appendix 1 represent the field-processed MT data at each station, and include some data scatter and poor signal-to-noise ratios. The only effort aimed at 
removing noisy data points was to visually inspect and select the best signal-to-noise field data to combine into the final data plots.

The impedance polar plots provide a measure of the MT data dimensionality (Reddy and others, 1977). For 1-D resistivity structures, the principal impedance (off-diagonal elements) polar diagram (dashed line) is a circle. For 2-D or 3-D resistivity structures, the principal impedance polar diagram (dashed line) elongates either parallel to or perpendicular to strike direction. Over resistors, the principal impedance polar diagram elongates perpendicular to strike direction, while over conductors, the principal impedance polar diagram elongates parallel to strike direction. For 2-D resistivity structures, the additional impedance polar diagram (solid line) attains the shape of a symmetric clover leaf. For 3-D resistivity structures, the additional impedance polar diagram (solid line) elongates in one direction, and its amplitude is comparable to that of the principal impedance polar diagram (dashed line), although high noise levels can produce the same effect on the polar diagram. A 3-D analysis of polar plots at each frequency should take into account the corresponding coherency and skew values along with their associated error levels. The polar plots computed for our data show station EB01 was 3-D below 0.06 Hz. Station EB02 was 3-D below 0.6 Hz. Station EB03 was 3-D over all frequencies.

The tipper can be calculated from the vertical component of the magnetic field. The tipper magnitude is a measure of the tipping of the magnetic field out of the horizontal plane (Vozoff, 1991). The magnitude is zero for the 1-D case, typically increases to between 0.1 to 0.5 , and rarely is as great as 1 , as it responds to vertical and subvertical structures. The tipper strike typically is used to help resolve the 90 -degree ambiguity in the impedance rotation angle. The tipper magnitude of these stations typically ranged between 0.1 and 0.6 over the lower frequencies, indicating some vertical structure at depth. The HzHx and HzHy coherency is a measure of the signal-to-noise ratio of the vertical magnetic field with respect to each of the orthogonal, horizontal magnetic field directions. Values are normalized between 0 and 1 , where values of 0.5 signify signal levels equal to noise levels. These three-components of magnetic-field coherence provide a check on the quality of the measured values in the tipper magnitude and tipper strike plots.

\section{References}

Alger, R.P., 1971, Interpretation of electric logs in fresh water wells in unconsolidated formations: Transactions of the Society of Professional Well Log Analysts $7^{\text {th }}$ Annual Logging Symposium, May 9-11, 1966, Houston, TX, 24 p.

Bendat, J.S., and Piersol, A.G., 1971, Random data-analysis and measurement procedures: New York, NY., Wiley Interscience, 407 p.

Deszcz-Pan, M., Rodriguez, B.D., Doucette, J.P., Godbout, M., Williams, J.M., Sawyer, D.A., Stone, B.D., and Grauch, V.J.S., 2000, Digital airborne time-domain electromagnetic data from surveys over Cochiti Pueblo, Rio Puerco, and Rio Rancho, New Mexico: U.S. Geological Survey Open-File Report 00-502 (CDROM). 
Dobrin, M.D., and Savit, C.H., 1988, Introduction to Geophysical Prospecting (4th ed.): New York, NY. McGraw-Hill, 867 p.

Eberhart-Phillips, Donna, Stanley, W.D., Rodriguez, B.D., and Lutter, W.J., 1995, Surface seismic and electrical methods to detect fluids related to faulting: Journal of Geophysical Research, v. 100, no. B7, p. 12,919-12,936.

EMI Inc., 1996, MT-1 magnetotelluric system operation manual, version 3.2: Richmond, Calif., Electromagnetic Instruments, Inc., 220 p.

Gamble, T.D., Goubau, W.M., and Clarke, J., 1979, Error analysis for remote reference magnetotellurics: Geophysics, v. 44, no. 5, p. 959-968.

Gilson, E.W., Nimeck, G., Bauman, P.D., and Kellett, R., 2000, Groundwater exploration in prairie environments, in Powers, M.H., Ibrahim, A., and Cramer, L., Proceedings of the Symposium on the Application of Geophysics to Environmental and Engineering Problems, p. 955-959.

Keller, G.V., 1987, Rock and mineral properties, in Nabighian, M.N., ed., Electromagnetic Methods in Applied Geophysics Theory: Tulsa, Okla., Society of Exploration Geophysicists, v. 1, p. 13-51.

Keller, G.V., 1989, Electrical properties, in Carmichael, R.S., ed., Practical Handbook of Physical Properties of Rocks and Minerals: Boca Raton, Fla., CRC Press, p. $359-427$.

Nelson, P.H., and Anderson, L.A., 1992, Physical properties of ash flow tuff from Yucca Mountain, Nevada: Journal of Geophysical Research, v. 97, no. B5, p. 6,823-6,841.

Olhoeft, G.R., 1989, Electrical properties of rocks, in Touloukian, Y.S., Judd, W.R., and Roy, R.F., eds., Physical Properties of Rocks and Minerals, CINDAS Data Series on Material Properties. Vol.II-2, Hemisphere Publishing, New York, NY, p. 257-329.

Palacky, G.J., 1987, Resistivity characteristics of geologic targets, in Nabighian, M.N., ed., Electromagnetic Methods in Applied Geophysics Theory: Tulsa, Okla., Society of Exploration Geophysicists, v. 1, p. 53-129.

Reddy, I.K., Rankin, David, and Phillips, R.J., 1977, Three-dimensional modelling in magnetotelluric and magnetic variational sounding: Geophysics Journal of the Royal Astronomical Society, v. 51, p. 313-325. 
Sternberg, B.K., Washburne, J.C., and Pellerin, Louise, 1988, Correction for the static shift in magnetotellurics using transient electromagnetic soundings: Geophysics, v. 53 , p. $1,459-1,468$.

Vozoff, Keeva, 1972, The magnetotelluric method in the exploration of sedimentary basins: Geophysics, v. 37, p. 98-141.

Vozoff, Keeva, 1991, The magnetotelluric method, in Nabighian, M.N., Electromagnetic Methods in Applied Geophysics: Tulsa, Oklahoma, Society of Exploration Geophysicists, v. 2, part B, p. 641-711.

Williams J.M., and Rodriguez, B.D., 2001, Magnetotelluric data in the Middle Rio Grande Basin at Cochiti Pueblo, New Mexico: U.S. Geological Survey Open-File Report 01-442, $66 \mathrm{p}$. (http://greenwood.cr.usgs.gov/pub/open-file-reports/ofr-01-0442/)

Williams J.M., and Rodriguez, B.D., 2003, Magnetotelluric data in the southwest Española Basin, northern New Mexico: U.S. Geological Survey Open-File Report 03-199, 128 p. (http://greenwood.cr.usgs.gov/pub/open-file-reports/ofr-03-0199/)

Williams, J.M., and Rodriguez, B.D., 2005a, Magnetotelluric data in the Española Basin west of Santa Fe, New Mexico: U.S. Geological Survey Open-File Report 2005-1037, 263 p. (http://pubs.usgs.gov/of/2005/1037/)

Williams, J.M., and Rodriguez, B.D., 2005b, Magnetotelluric data along the Pajarito Fault in the Española Basin west of Santa Fe, New Mexico: U.S. Geological Survey Open-File Report 2005-1054, 173 p. (http://pubs.usgs.gov/of/2005/1052/) 


\section{Appendix 1}

\section{Magnetotelluric Data Plots}

There are nine separate plots for each station:

1. Apparent Resistivity for the unrotated xy (x symbol) and yx (o symbol) modes

2. Impedance Phase for the unrotated $x y$ ( $x$ symbol) and yx (o symbol) modes

3. Rotation Angle for the impedance tensor (corresponds to the direction of $x y$ component)

4. Impedance Skew for the impedance tensor

5. Multiple Coherency for the xy (x symbol) and minimum (o symbol) modes of the electric field

6. Impedance Polar Plots (at 12 selected frequencies)

7. Tipper Magnitude for the vertical magnetic field

8. Tipper Strike for the vertical magnetic field

9. HzHx (x symbol) and HzHy (o symbol) Coherency

Refer to the "Magnetotelluric Data" section in this report for an explanation of these plots. 


\section{Station EB01}

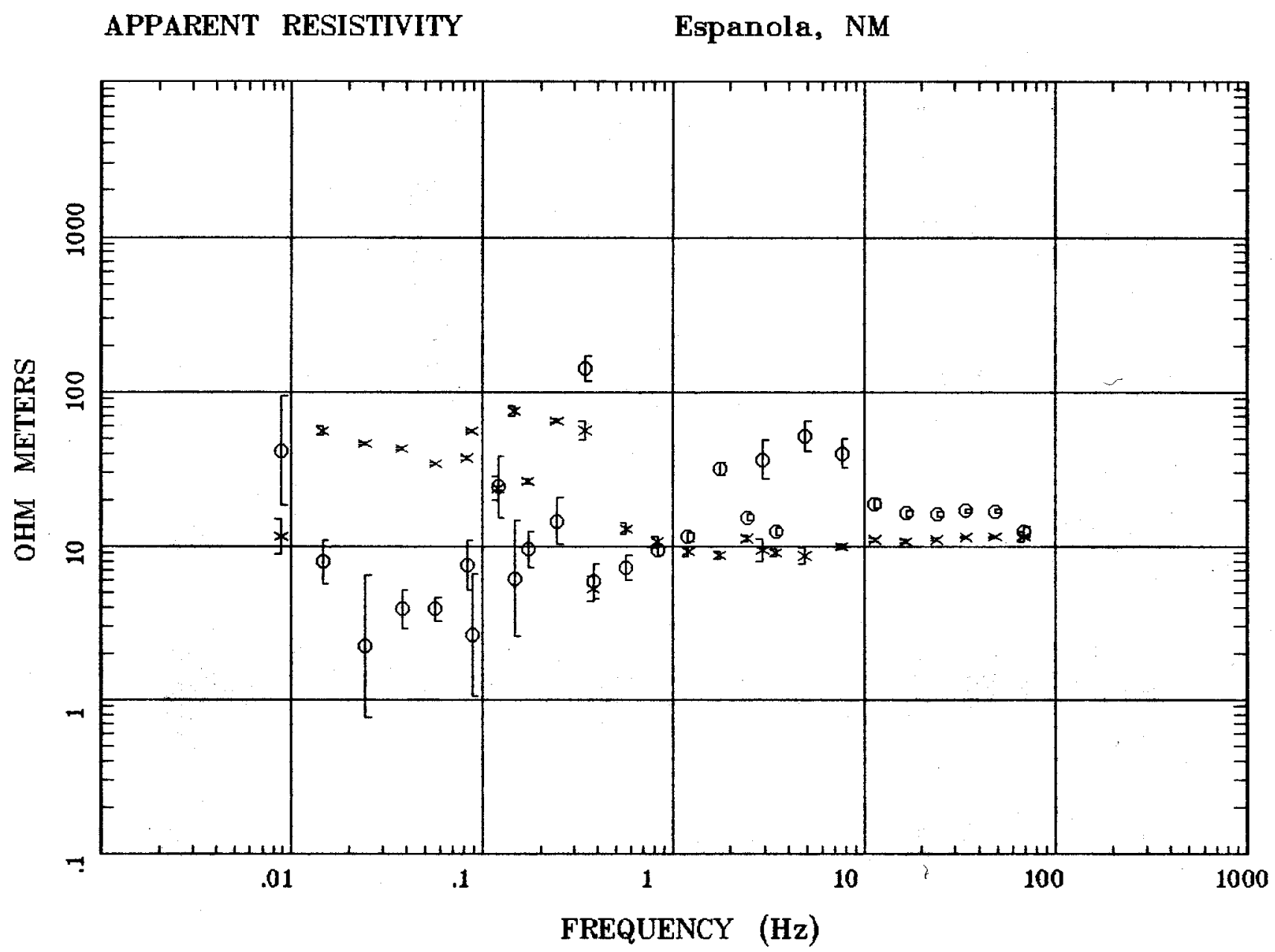

Client:

Remote: e-fld $95 \mathrm{~m}$ north Acquired: 10:4 Jul 22, 2002 Survey Co:USGS
Rotation:

Filename: eb01a.avg

Channels: Ch1 Ch2 Ch3 Ch4 Ch5 Ch6 Ch7

Plotted: 09:11 Mar 12, 2003

< EMI - ElectroMagnetic Instruments > 




Client:

Remote: e-fld $95 \mathrm{~m}$ north Acquired: 10:4 Jul 22, 2002 Survey Co:USGS
Rotation:

Filename: eb01a.avg

Channels: Ch1 Ch2 Ch3 Ch4 Ch5 Ch6 Ch7 Plotted: 09:11 Mar 12, 2003

$<\quad$ EMI - ElectroMagnetic Instruments 
Station EB01

ROTATION ANGLE

Espanola, NM

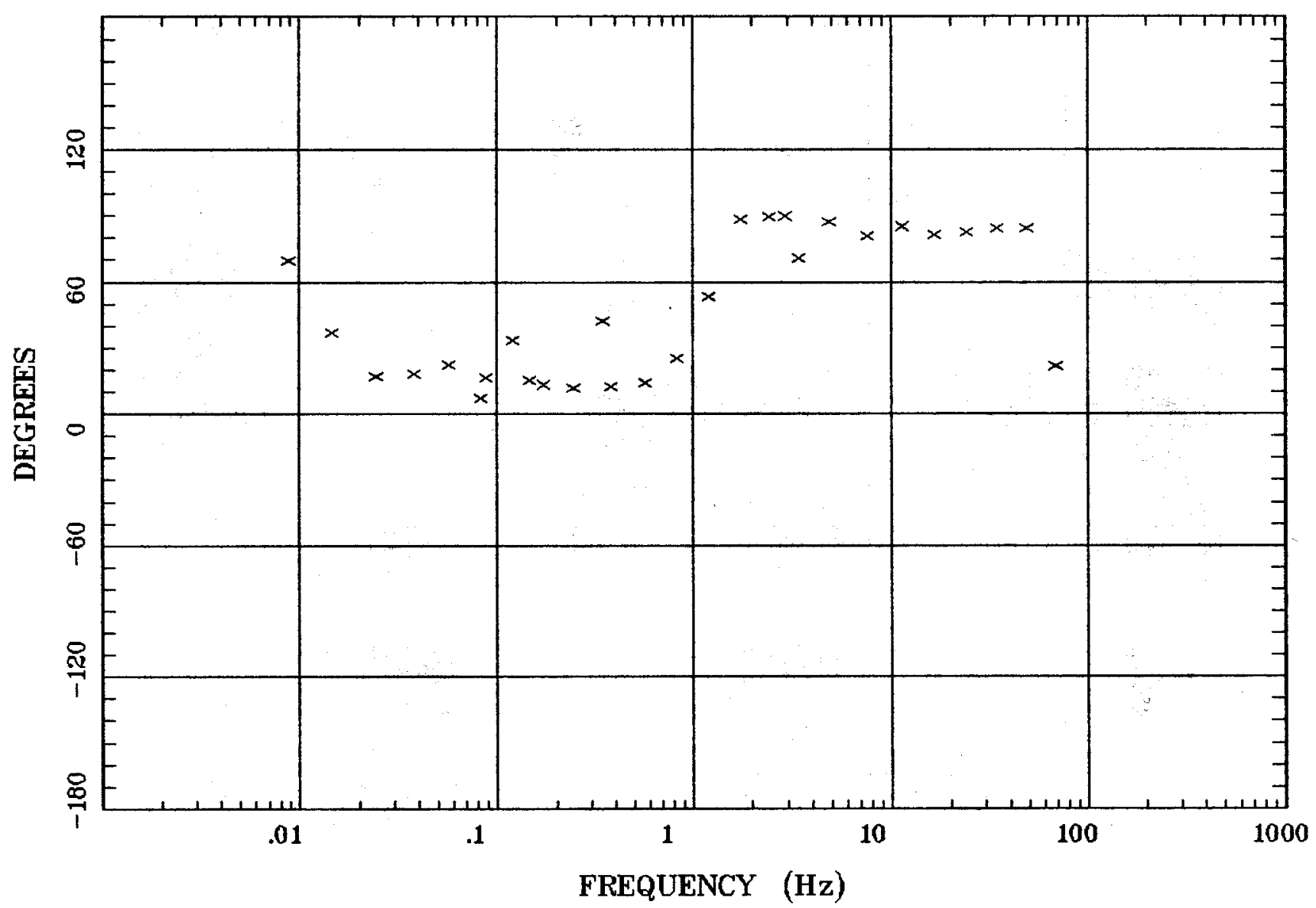

Client:

Remote: e-fld $95 \mathrm{~m}$ north Acquired: 10:4 Jul 22, 2002 Survey Co:USGS
Rotation:

Filename: eb01a.avg

Channels: Ch1 Ch2 Ch3 Ch4 Ch5 Ch6 Ch7 Plotted: 09:11 Mar 12, 2003

< EMI - ElectroMagnetic Instruments 


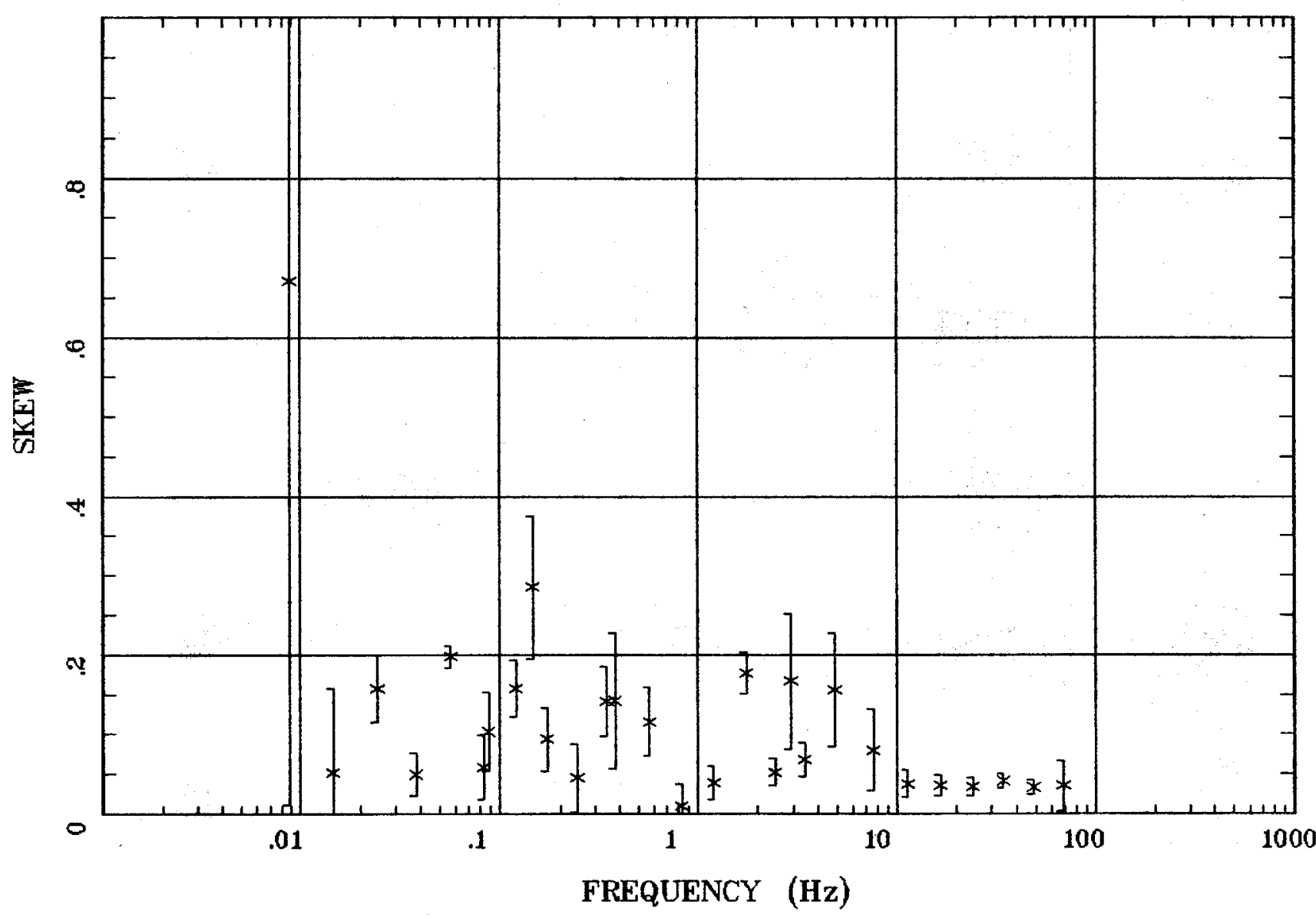

Client:

Remote: e-fld $95 \mathrm{~m}$ north Acquired: 10:4 Jul 22, 2002 Survey Co:USGS
Rotation:

Filename: eb01a.avg

Channels: Ch1 Ch2 Ch3 Ch4 Ch5 Ch6 Ch7 Plotted: 09:11 Mar 12, 2003

< EMI - ElectroMagnetic Instruments 
Station EB01

E MULT Coh.



Client:

Remote: e-fld $95 \mathrm{~m}$ north Acquired: 10:4 Jul 22, 2002 Survey Co:USGS

Espanola, NM

Rotation:

Filename: eb01a.avg

Channels: Ch1 Ch2 Ch3 Ch4 Ch5 Ch6 Chy

Plotted: 09:11 Mar 12, 2003

< EMI - ElectroMagnetic Instruments > 
Station EB01



Client:

Remote: e-fld $95 \mathrm{~m}$ north Acquired: 10:4 Jul 22, 2002 Survey Co:USGS
Rotation:

Filename: eb01a.avg

Channels: Ch1 Ch2 Ch3 Ch4 Ch5 Ch6 Chr

Platted: 09:12 Mar 12, 2003

$<$ EMI - ElectroMagnetic Instruments > 


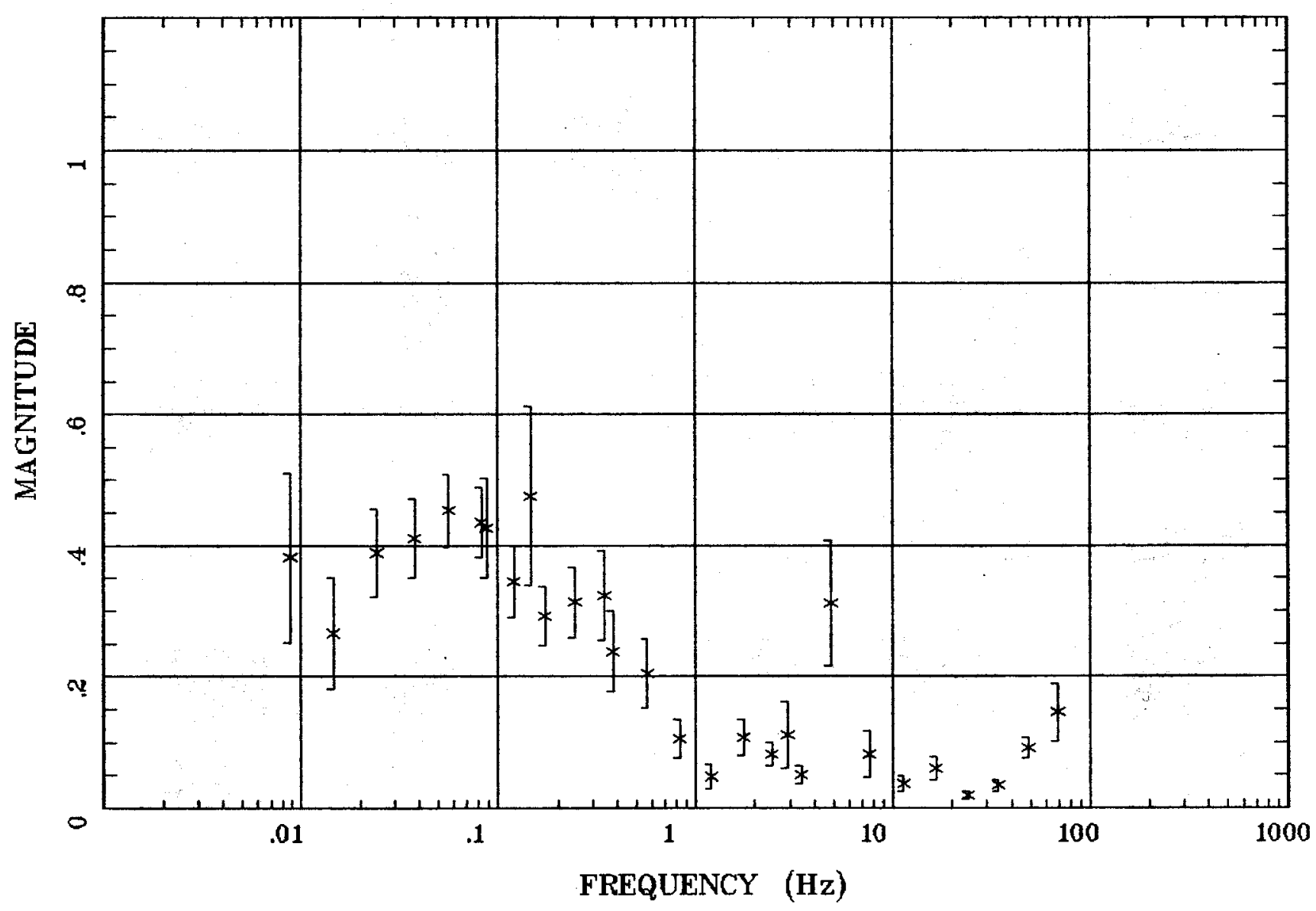

Client:

Remote: e-fld $95 \mathrm{~m}$ north Acquired: 10:4 Jul 22, 2002 Survey Co:USGS
Rotation:

Filename: eb01a.avg

Channels: Ch1 Ch2 Ch3 Ch4 Ch5 Ch6 Ch7 Plotted: 09:12 Mar 12, 2003

< EMI - ElectroMagnetic Instruments > 


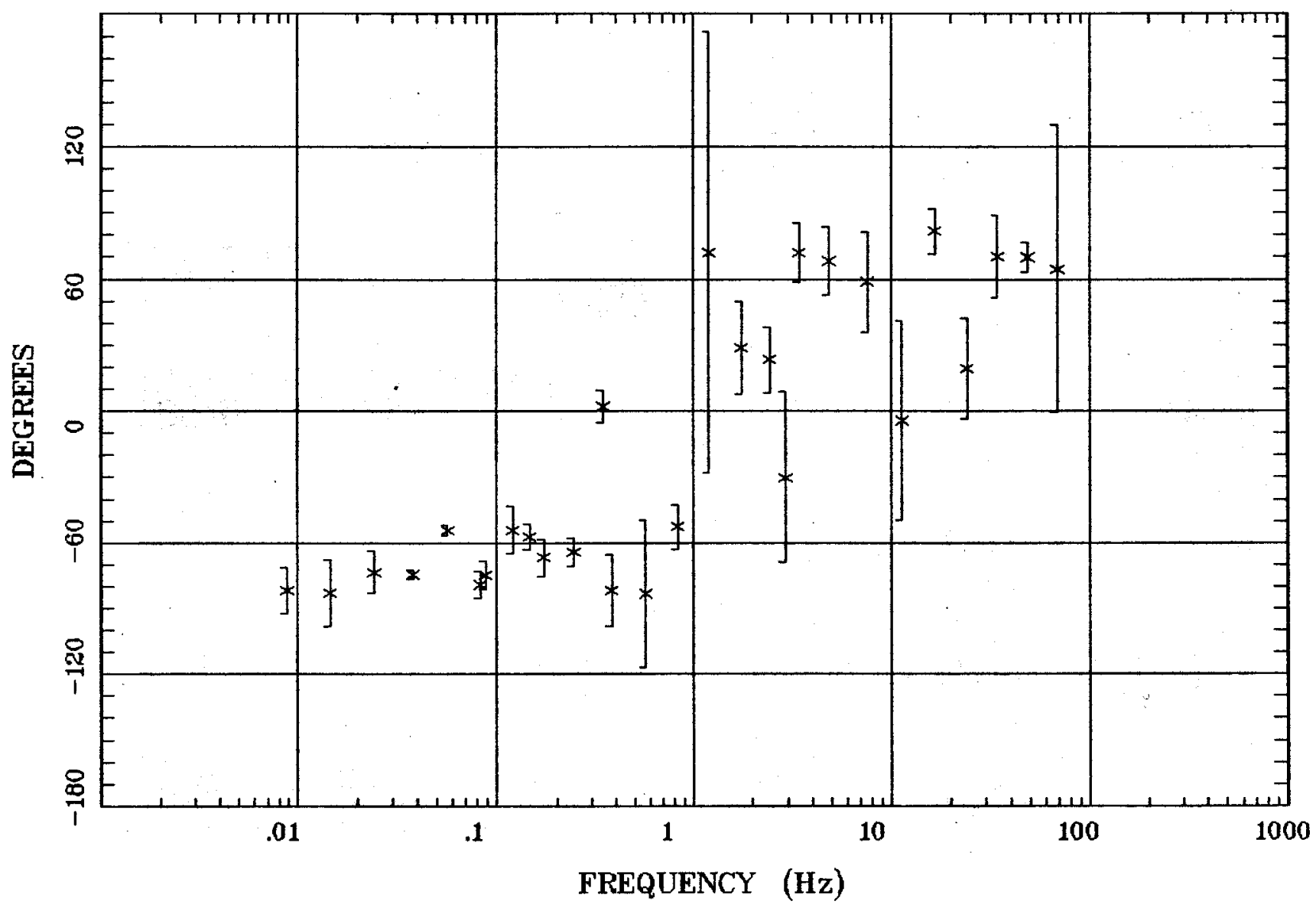

Client:

Remote: e-fld $95 \mathrm{~m}$ north Acquired: 10:4 Jul 22, 2002 Survey Co:USGS
Rotation:

Filename: eb01a.avg

Channels: Ch1 Ch2 Ch3 Ch4 Ch5 Ch6 Ch7 Plotted: 09:13 Mar 12, 2003

< EMI - ElectroMagnetic Instruments 
HzHx.x Coh HzHy.o



Client:

Remote: e-fld $95 \mathrm{~m}$ north Acquired: 10:4 Jul 22, 2002 Survey Co:USGS

Espanola, NM

Station EB01

Rotation:

Filename: eb01a.avg Channels: Ch1 Ch2 Ch3 Ch4 Ch5 Ch6 Ch' Plotted: 09:13 Mar 12, 2003

< EMI - ElectroMagnetic Instruments > 


\section{Station EB02}

APPARENT RESISTIVITY

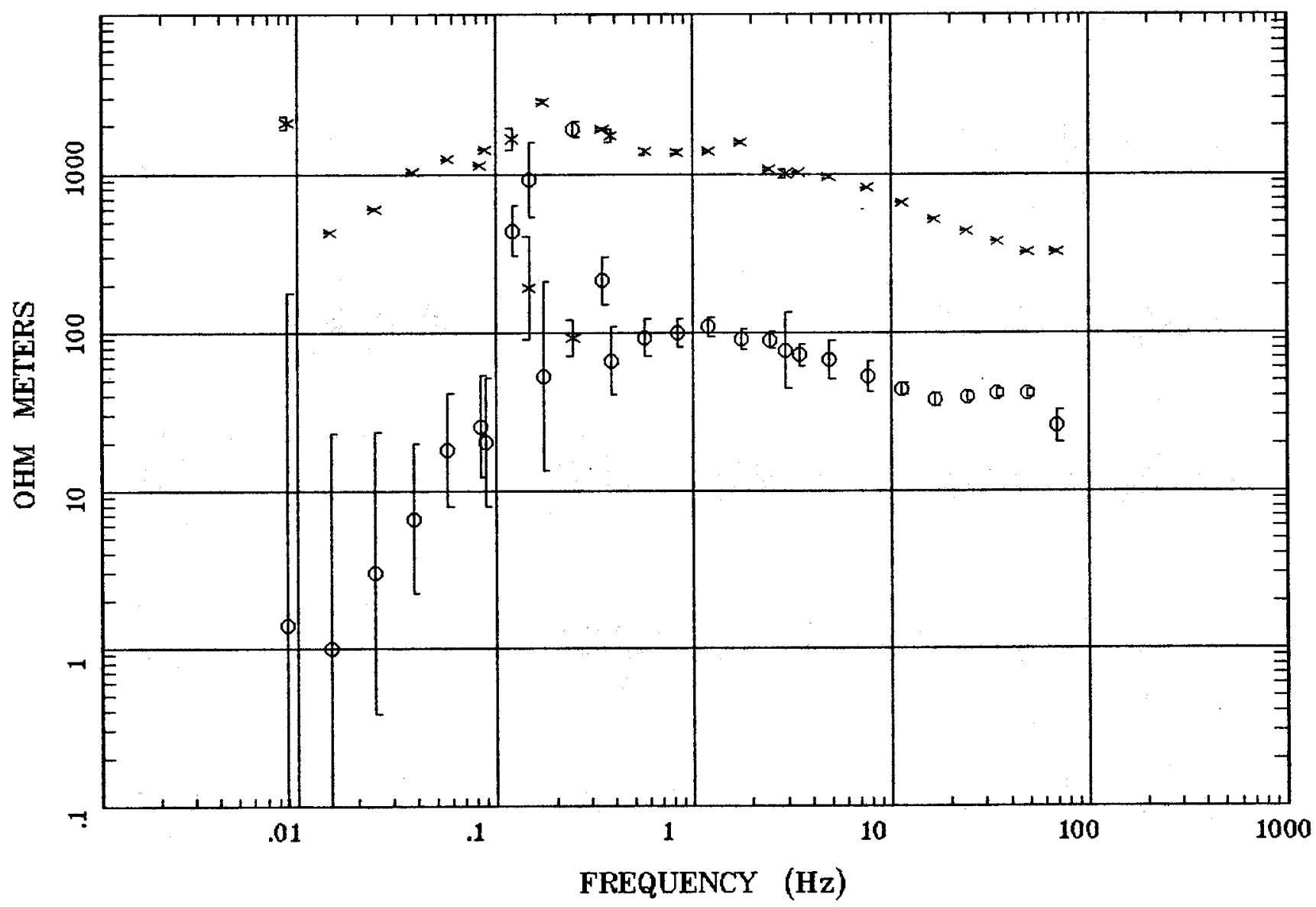

Client:

Remote: e-fld $95 \mathrm{~m}$ west Acquired: 12:2 Jul 28, 2002 Survey Co:USGS
Rotation:

Filename: eb02b.avg Channels: Ch1 Ch2 Ch3 Ch4 Ch5 Ch6 Ch7 Plotted: 09:14 Mar 12, 2003

< EMI - ElectroMagnetic Instruments > 
IMPEDANCE PHASE

Espanola, NM

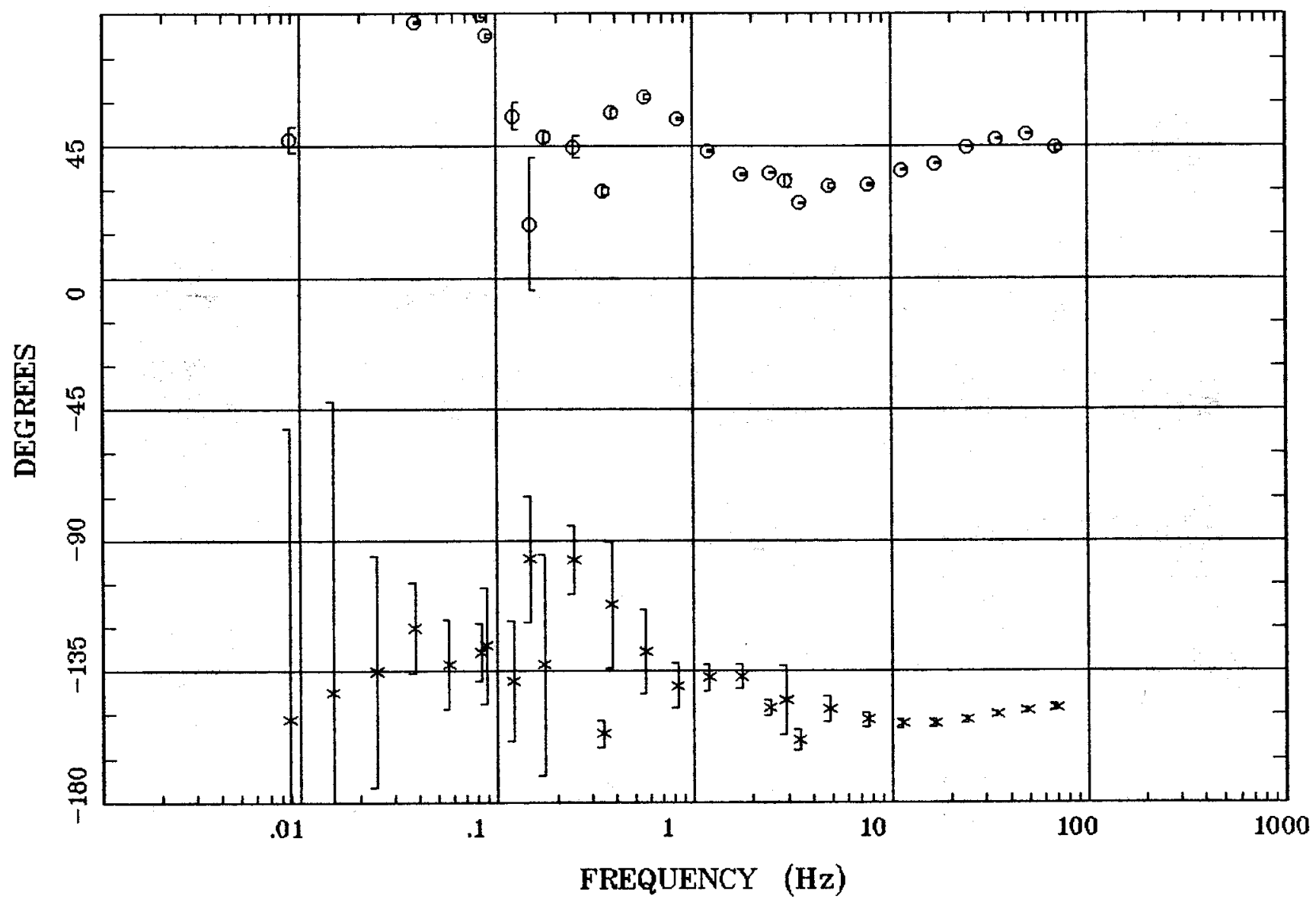

Client:

Remote: e-fld $95 \mathrm{~m}$ west Acquired: 12:2 Jul 28, 2002 Survey Co:USGS
Rotation:

Filename: eb02b.avg

Channels: Ch1 Ch2 ch3 ch4 Ch5 ch6 Ch'

Plotted: 09:14 Mar 12, 2003

< EMI - ElectroMagnetic Instruments > 


\section{ROTATION ANGLE Espanola, NM}

Station EB02

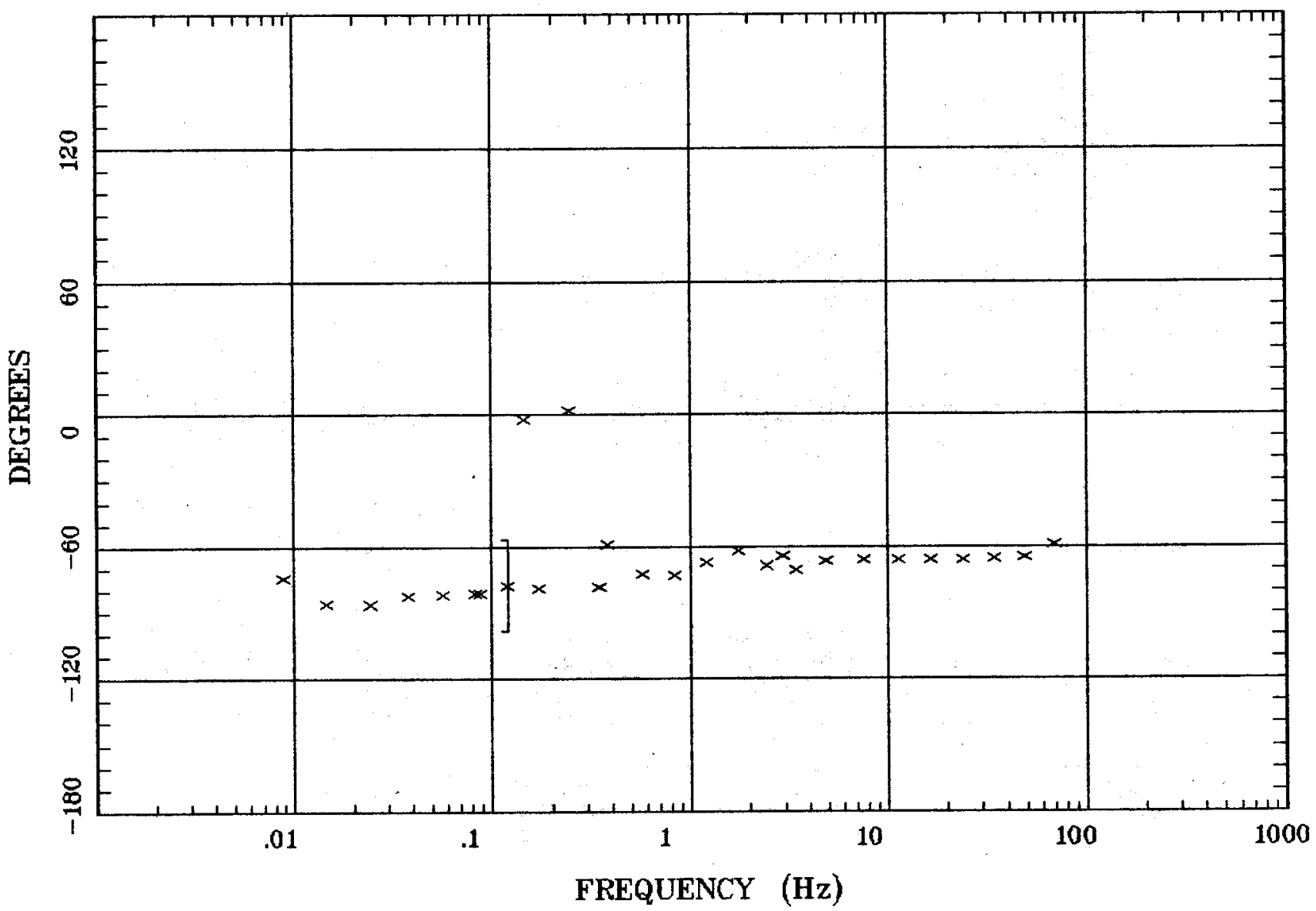

Client:

Remote: e-fld $95 \mathrm{~m}$ west

Acquired: 12:2 Jul 28, 2002

Survey Co:USGS
Rotation:

Filename: eb02b.avg

Channels: Ch1 Ch2 Ch3 Ch4 Ch5 Ch6 Ch7 Plotted: 09:14 Mar 12, 2003

< EMI - ElectroMagnetic Instruments > 


\section{Station EB02}

IMPEDANCE SKEW

Espanola, NM

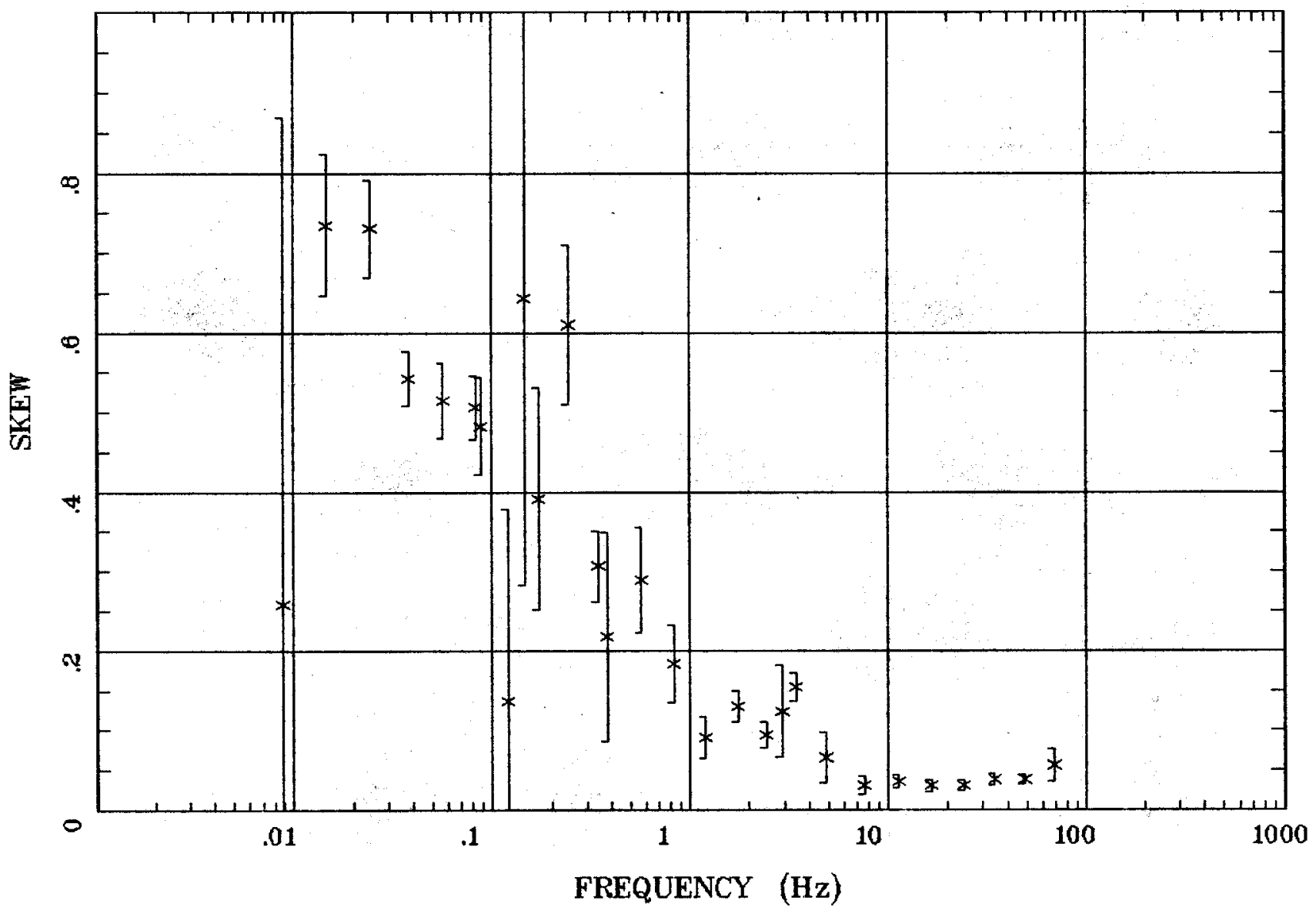

Client:

Remote: e-fld $95 \mathrm{~m}$ west

Acquired: 12:2 Jul 28, 2002

Rotation:

Filename: eb02b.avg

Channels: Ch1 Ch2 Ch3 Ch4 Ch5 Ch6 Ch7

Plotted: 09:14 Mar 12, 2003

Survey Co:USGS

$<$ EMI - ElectroMagnetic Instruments 


\section{Station EB02}

E MULT Coh.

Espanola, NM

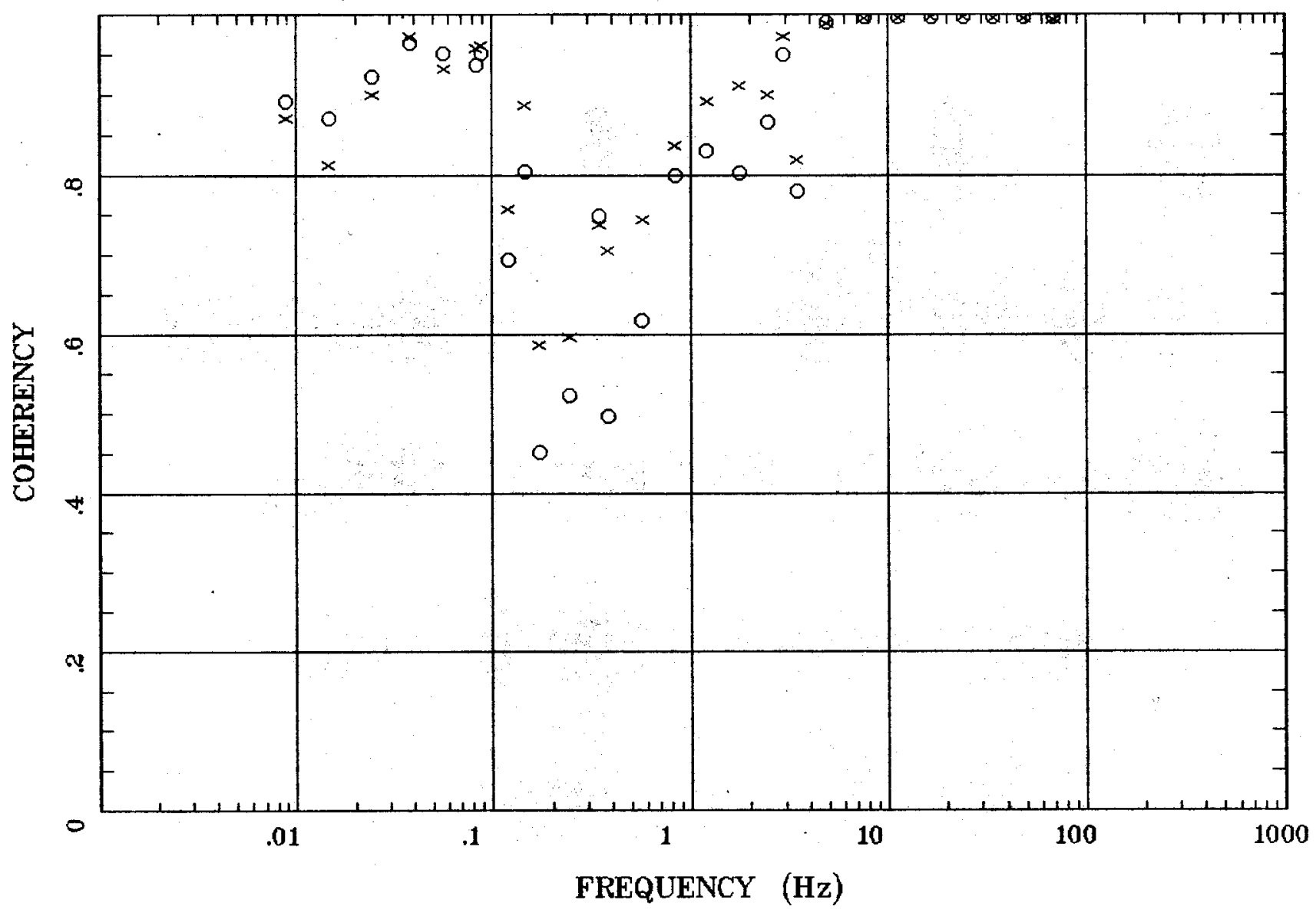

Client:

Remote: e-fld $95 \mathrm{~m}$ west

Acquired: 12:2 Jul 28, 2002

Survey Co:USGS
Rotation:

Filename: eb02b.avg

Channels: Ch1 Ch2 Ch3 Ch4 Ch5 Ch6 Ch7

Plotted: 09:14 Mar 12, 2003

< EMI - ElectroMagnetic Instruments 


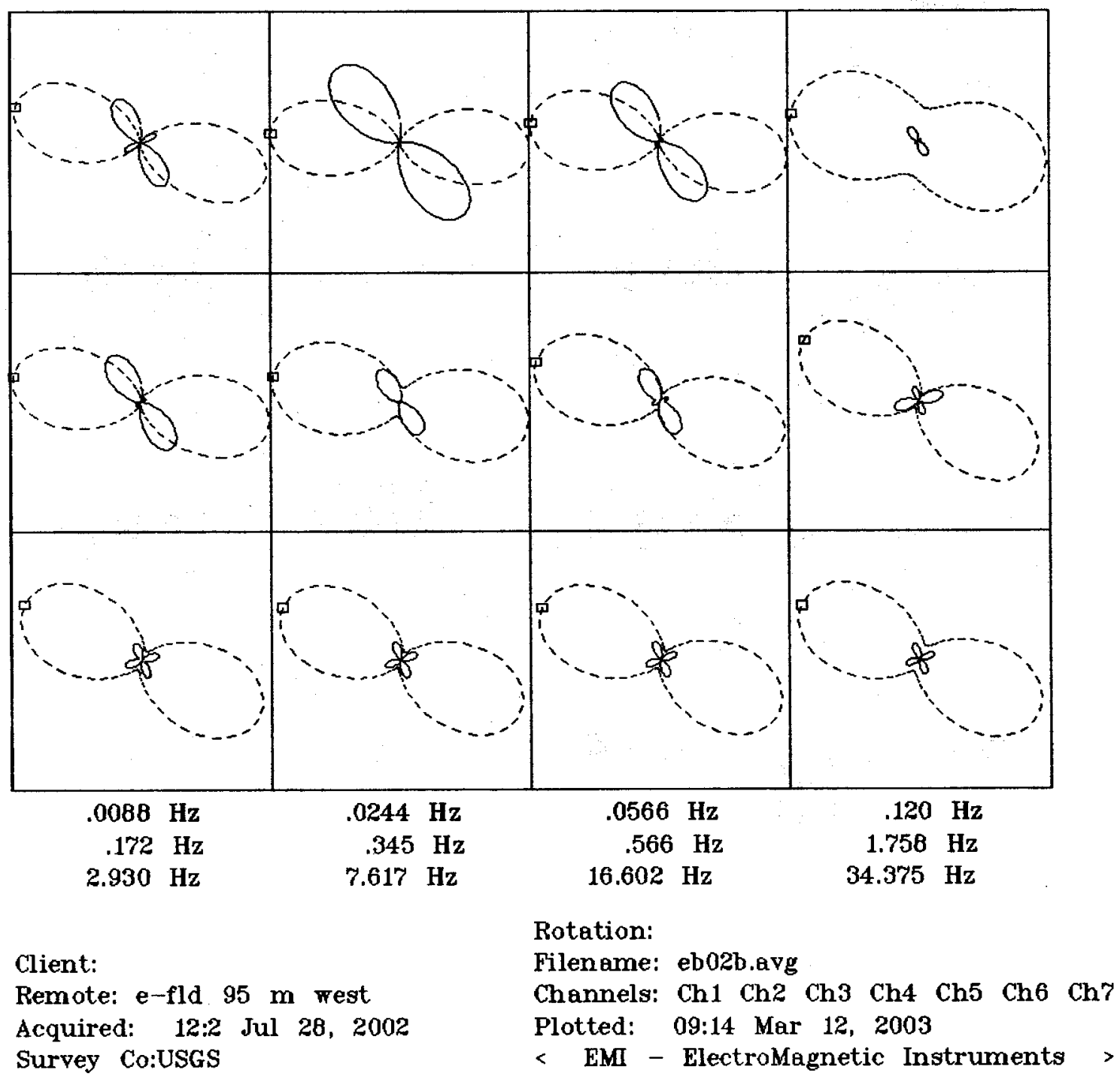






Client:

Remote: e-fld $95 \mathrm{~m}$ west Acquired: 12:2 Jul 28, 2002 Survey Co:USGS

\section{Rotation:}

Filename: eb02b.avg

Channels: Ch1 Ch2 Ch3 Ch4 Ch5 Ch6 Ch7 Plotted: 09:14 Mar 12, 2003

< EMI - ElectroMagnetic Instruments 
TIPPER STRIKE

Espanola, NM

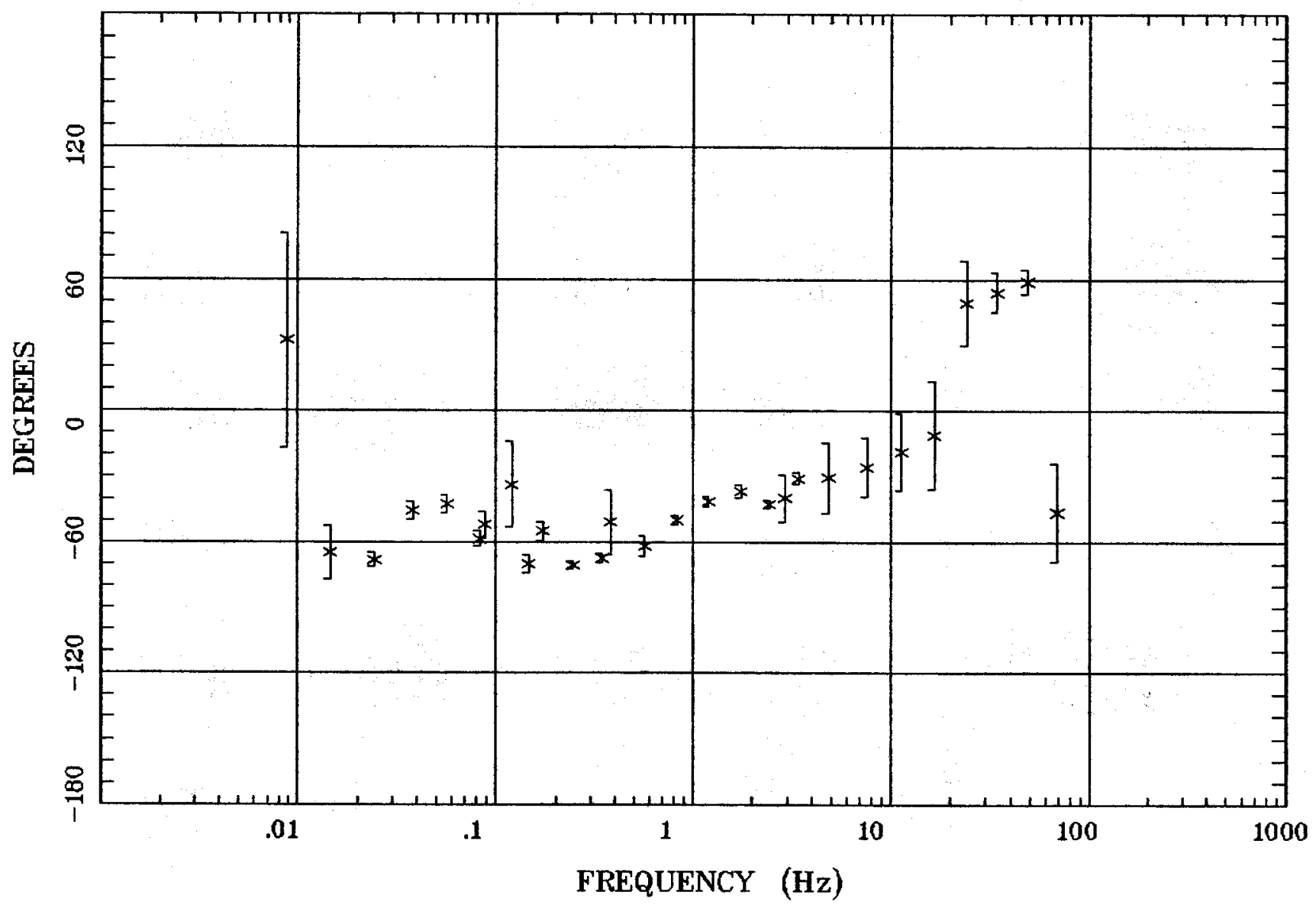

Client:

Remote: e-fld $95 \mathrm{~m}$ west Acquired: 12:2 Jul 28, 2002 Survey Co:USGS
Rotation:

Filename: eb02b.avg

Channels: Ch1 Ch2 Ch3 Ch4 Ch5 Ch6 Ch7

Plotted: 09:14 Mar 12, 2003

$<$ EMI - ElectroMagnetic Instruments 
Station EB02

HzHx.x Coh HzHy.o

Espanola, NM

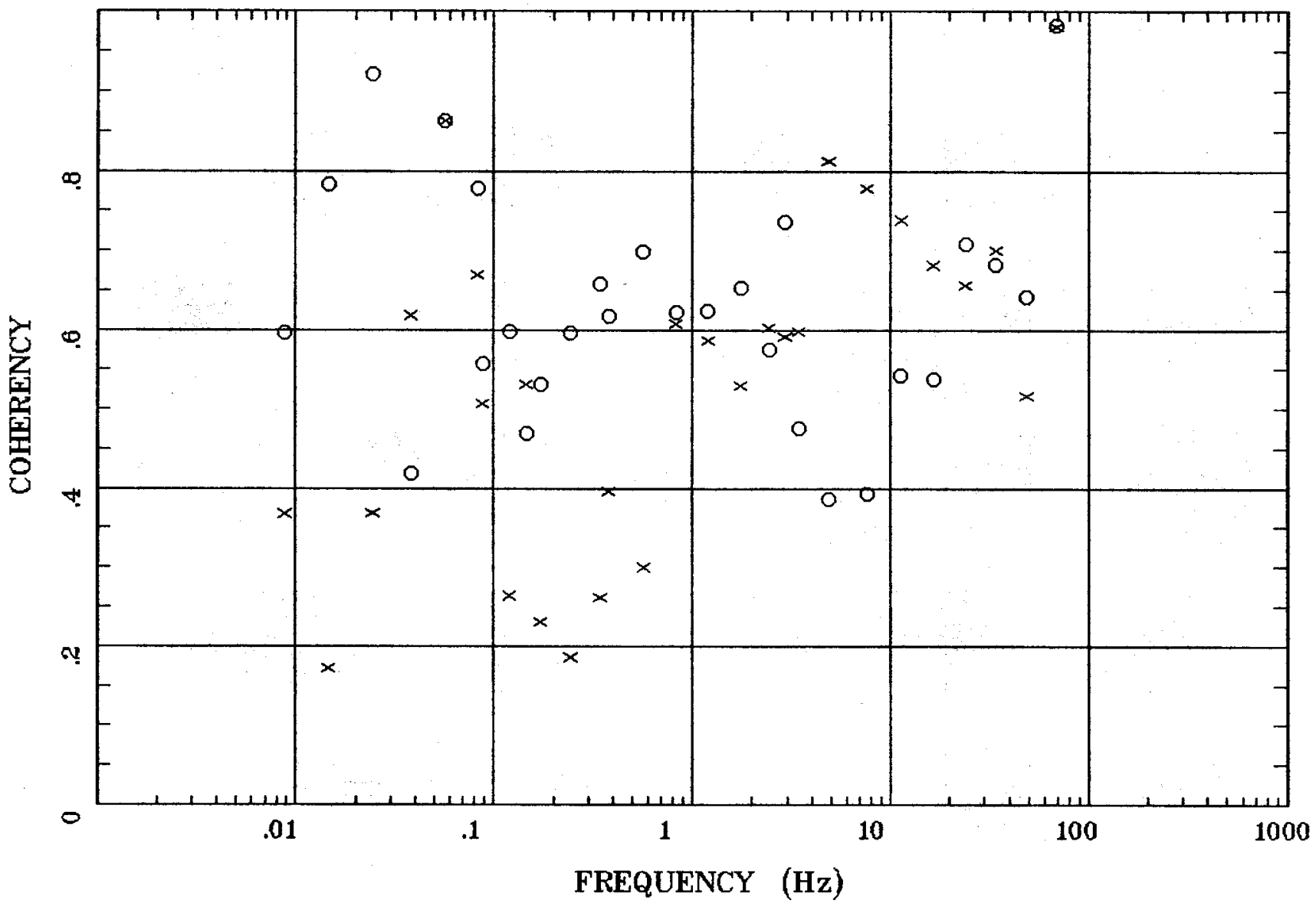

Client:

Remote: e-fld $95 \mathrm{~m}$ west Acquired: 12:2 Jul 28, 2002 Survey Co:USGS
Rotation:

Filename: eb02b.avg

Channels: Ch1 Ch2 Ch3 Ch4 Ch5 Ch6 Ch7 Plotted: 09:14 Mar 12, 2003

< EMI - ElectroMagnetic Instruments > 


\section{APPARENT RESISTIVITY}

Espanola, NM

Station EB03

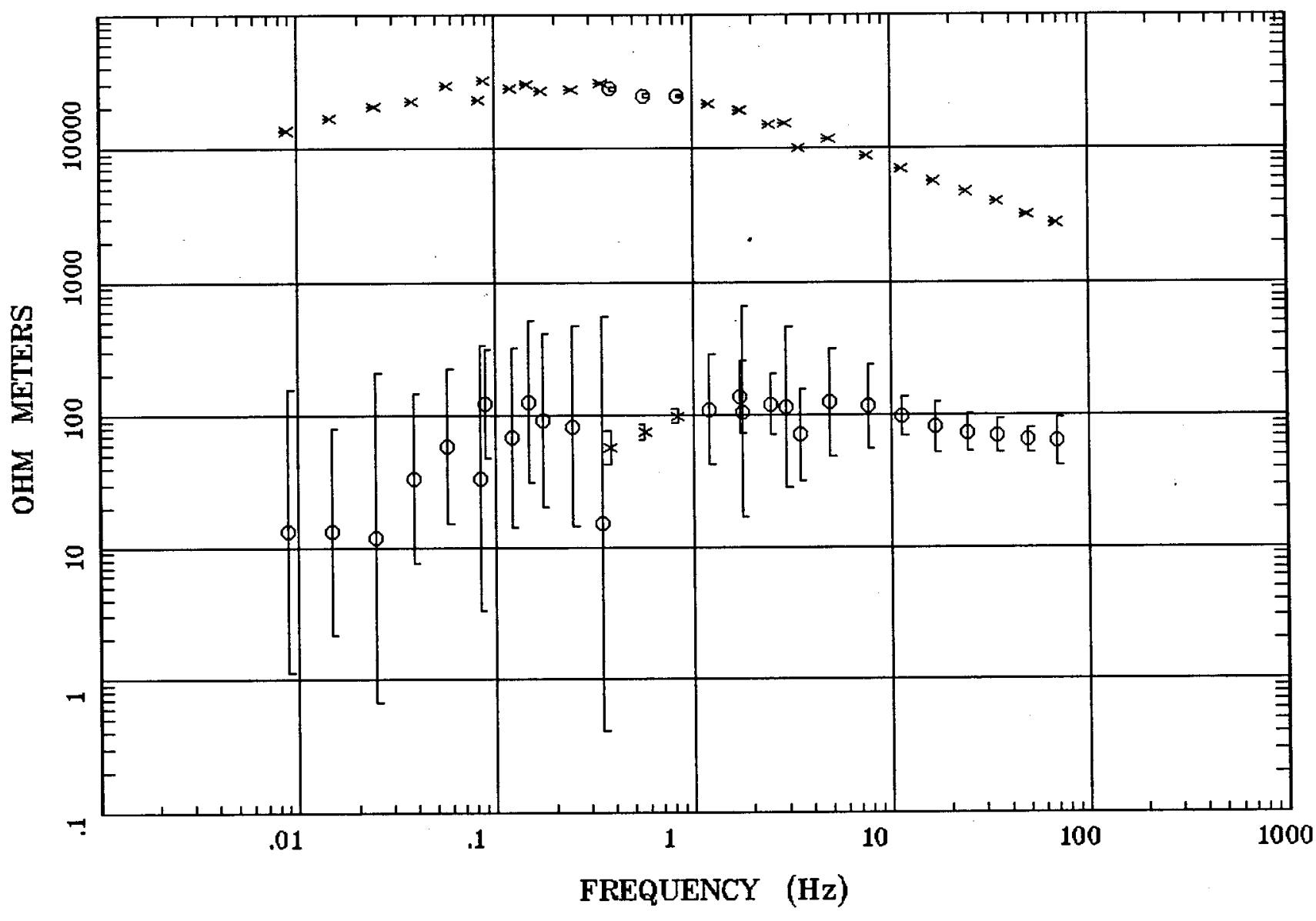

Client:

Remote: none

Acquired: 16:3 Jul 28, 2002 Survey Co:USGS
Rotation:

Filename: eb03all.avg

Channels: Ch1 Ch2 Ch3 Ch4 Ch5 Ch3 Ch4 Plotted: 09:55 Jan 11, 2006

$<$ EMI - ElectroMagnetic Instruments 
IMPEDANCE PHASE

Espanola, NM

Station EB03

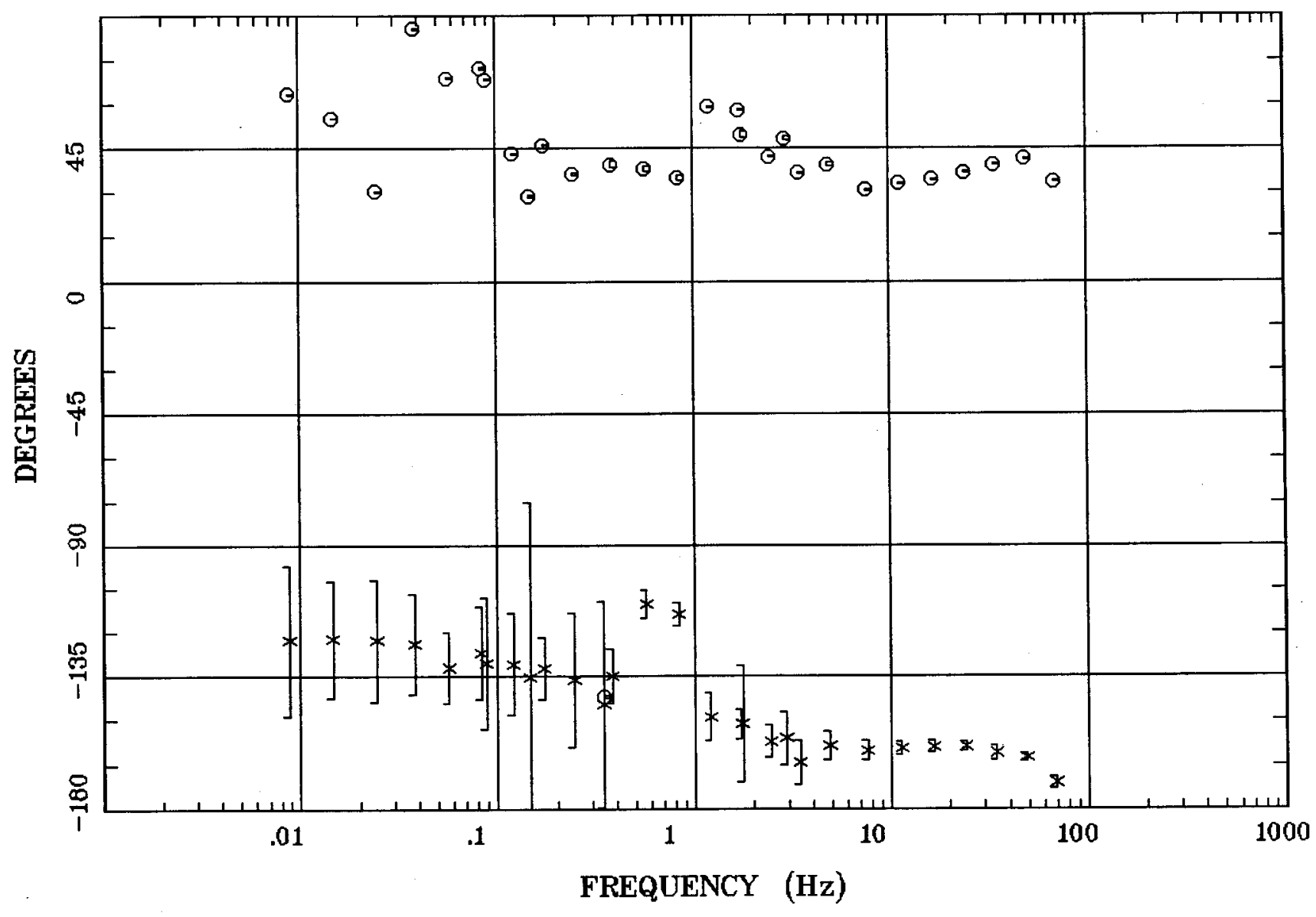

Client:

Remote: none

Acquired: 16:3 Jul 28, 2002 Survey Co:USGS
Rotation:

Filename: eb03all.avg

Channels: Ch1 Ch2 Ch3 Ch4 Ch5 Ch3 Ch4 Plotted: 09:55 Jan 11, 2006

$<$ EMI - ElectroMagnetic Instruments 


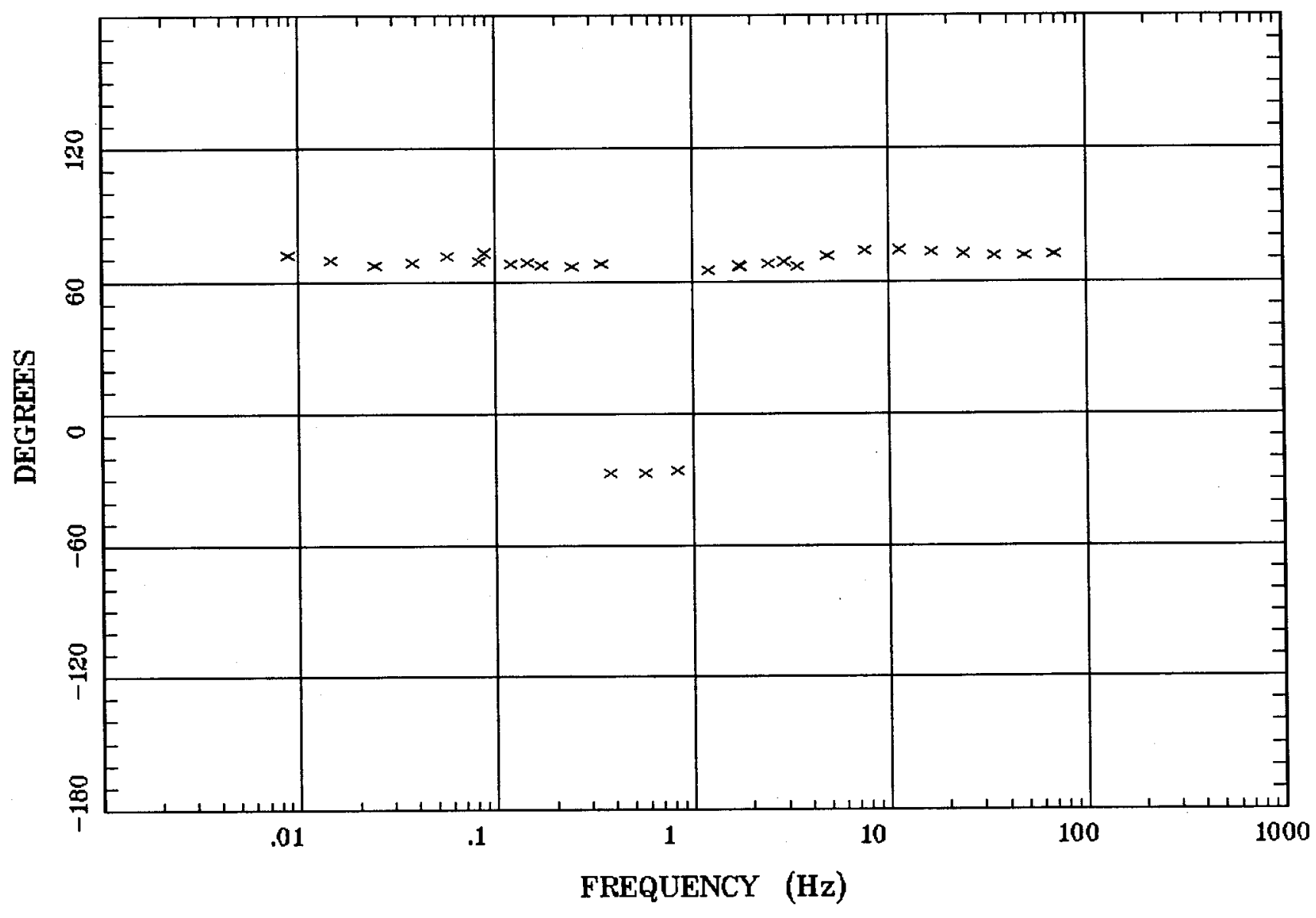

Client:

Remote: none

Acquired: $16: 3$ Jul 28, 2002 Survey Co:USGS
Rotation:

Filename: eb03all.avg

Channels: Ch1 Ch2 Ch3 Ch4 Ch5 Ch3 Ch4 Plotted: 09:55 Jan 11, 2006

< EMI - ElectroMagnetic Instruments 


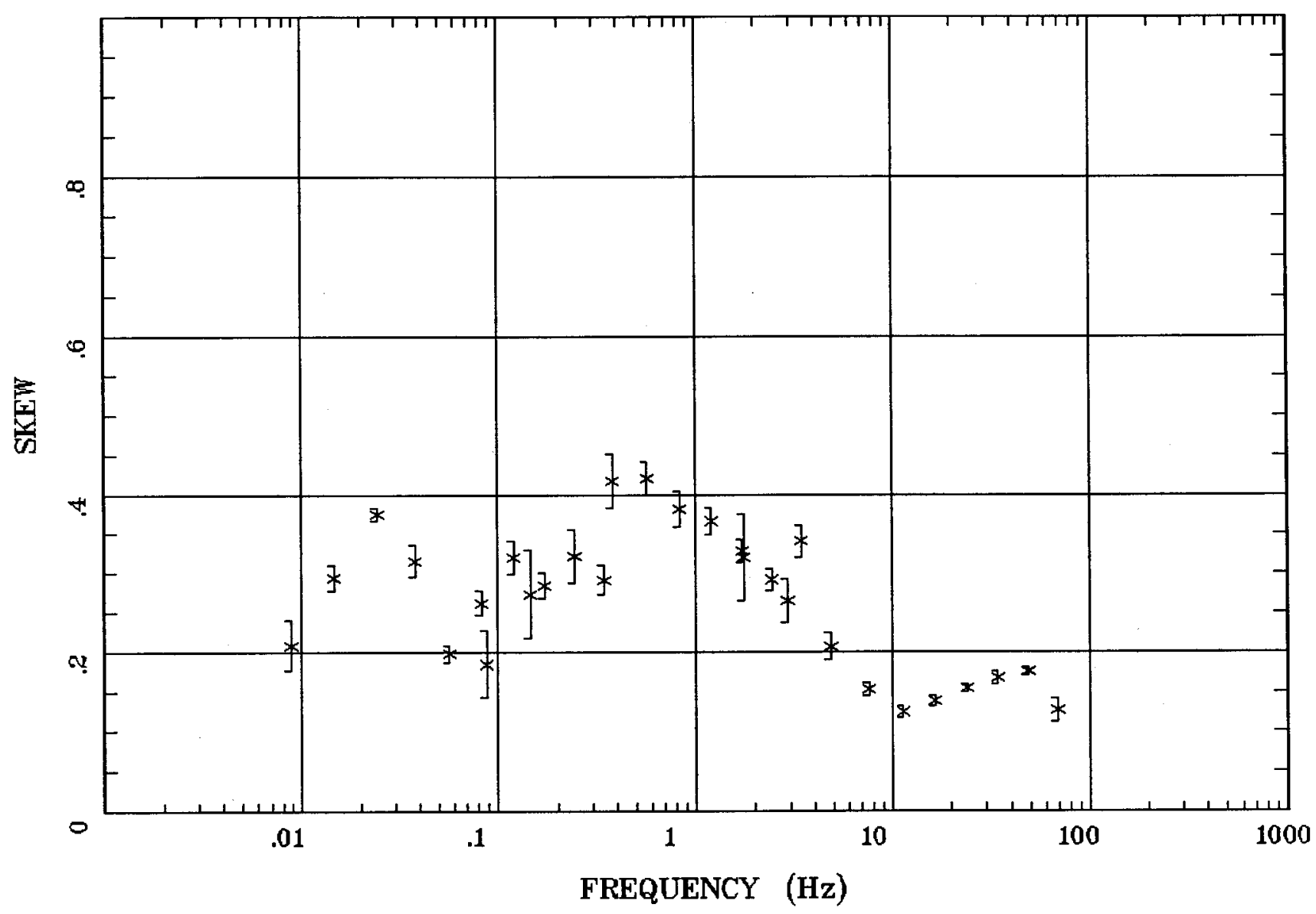

Client:

Remote: none

Acquired: 16:3 Jul 28, 2002

Survey Ca:USGS
Rotation:

Filename: eb03all.avg

Channels: Ch1 Ch2 Ch3 Ch4 Ch5 ch3 Ch4 Plotted: 09:55 Jan 11, 2006

$<$ EMI - ElectroMagnetic Instruments 


\section{E MULT Coh.}

Espanola, NM

Station EB03

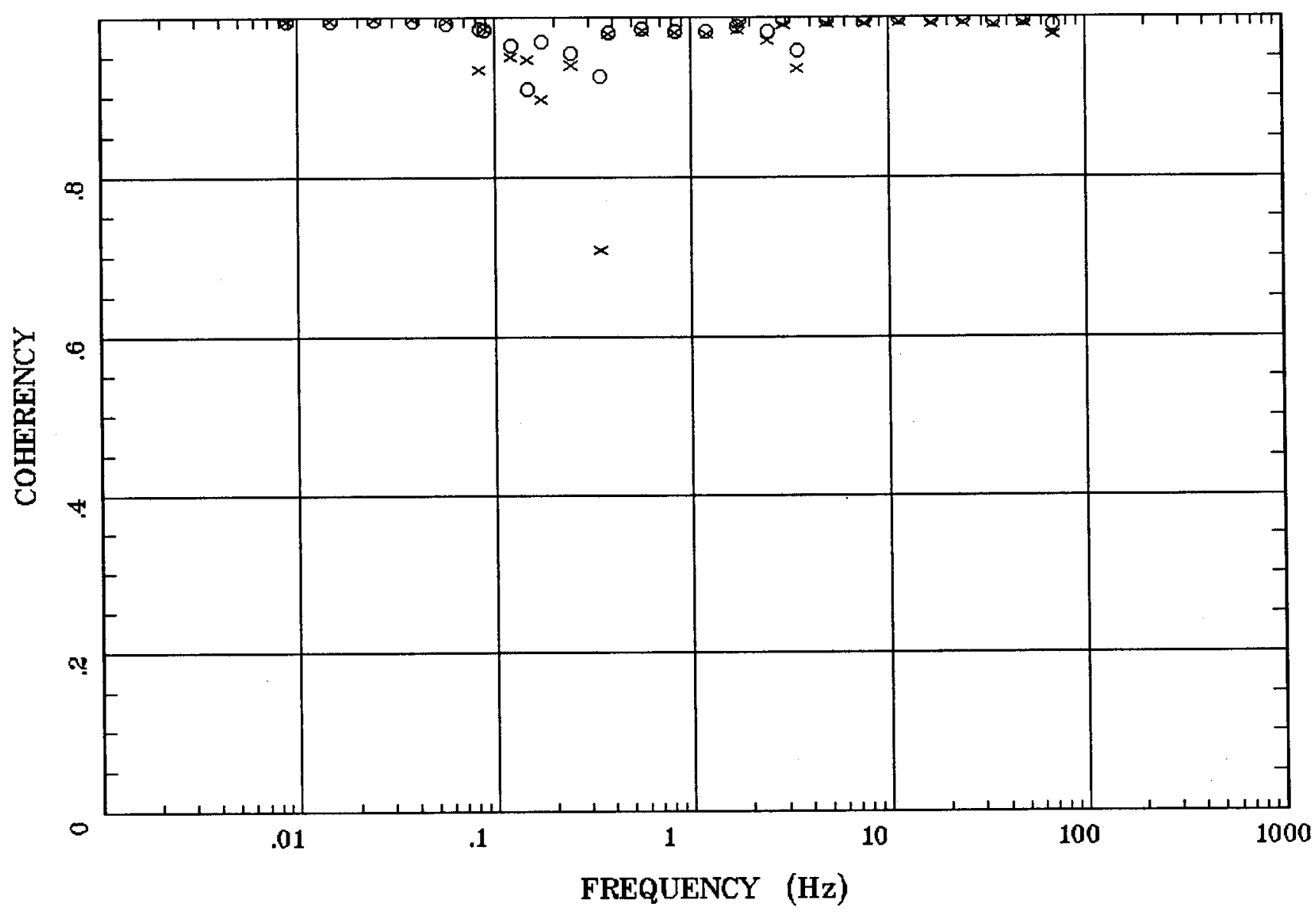

Client:

Remote: none

Acquired: $16: 3$ Jul 28, 2002

Survey Co:USGS
Rotation:

Filename: eb03all.avg

Channels: Ch1 Ch2 Ch3 Ch4 Ch5 Ch3 Ch4

Plotted: 09:55 Jan 11, 2006

$<$ EMI - ElectroMagnetic Instruments > 


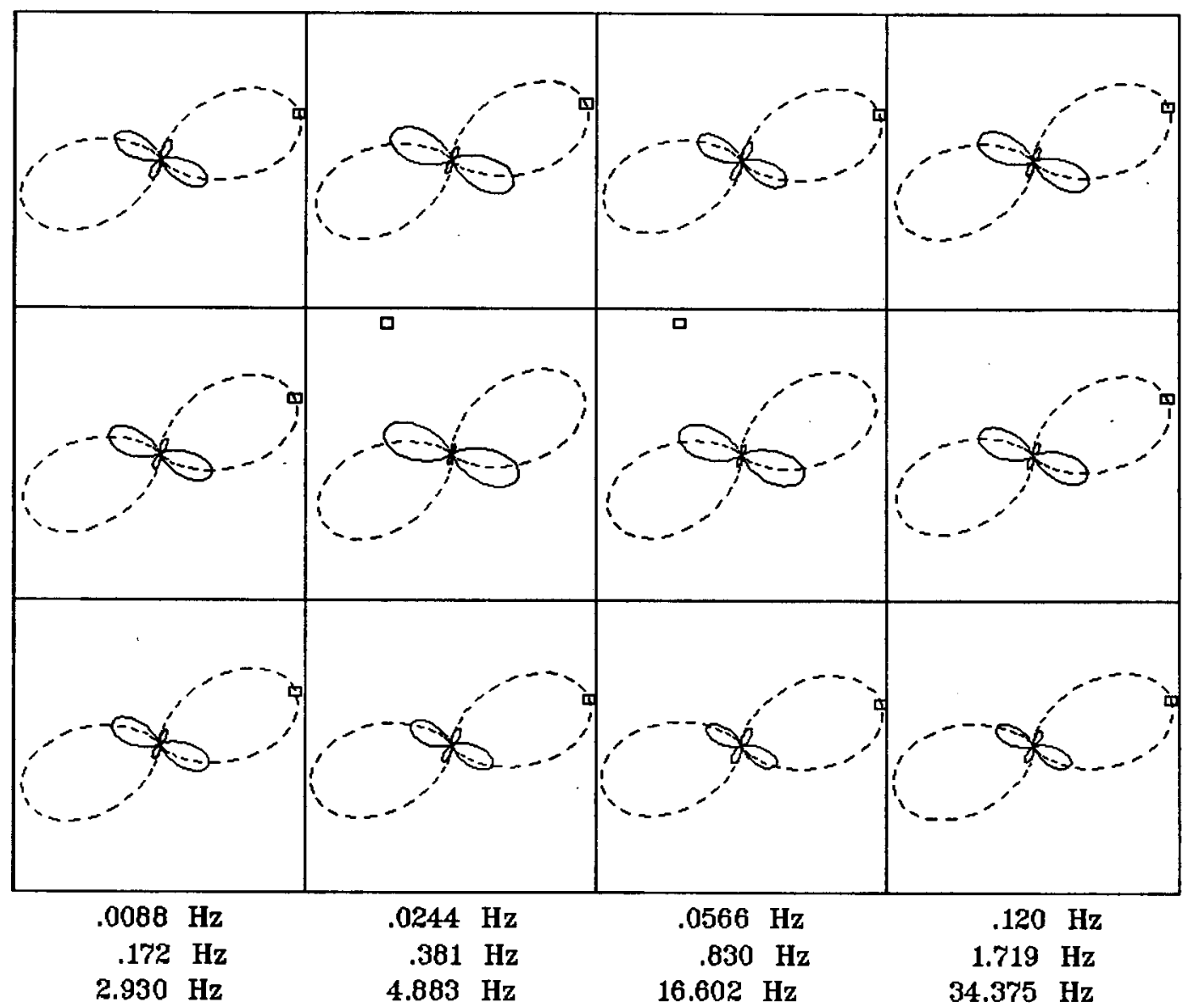

Client:

Remote: none

Acquired: 16:3 Jul 28, 2002 Survey Co:USGS
Rotation:

Filename: eb03all.avg

Channels: Ch1 Ch2 Ch3 Ch4 Ch5 Ch3 Ch4 Plotted: 09:55 Jan 11, 2006

< EMI - ElectroMagnetic Instruments > 


\section{TIPPER MAGNITUDE}

Espanola, NM

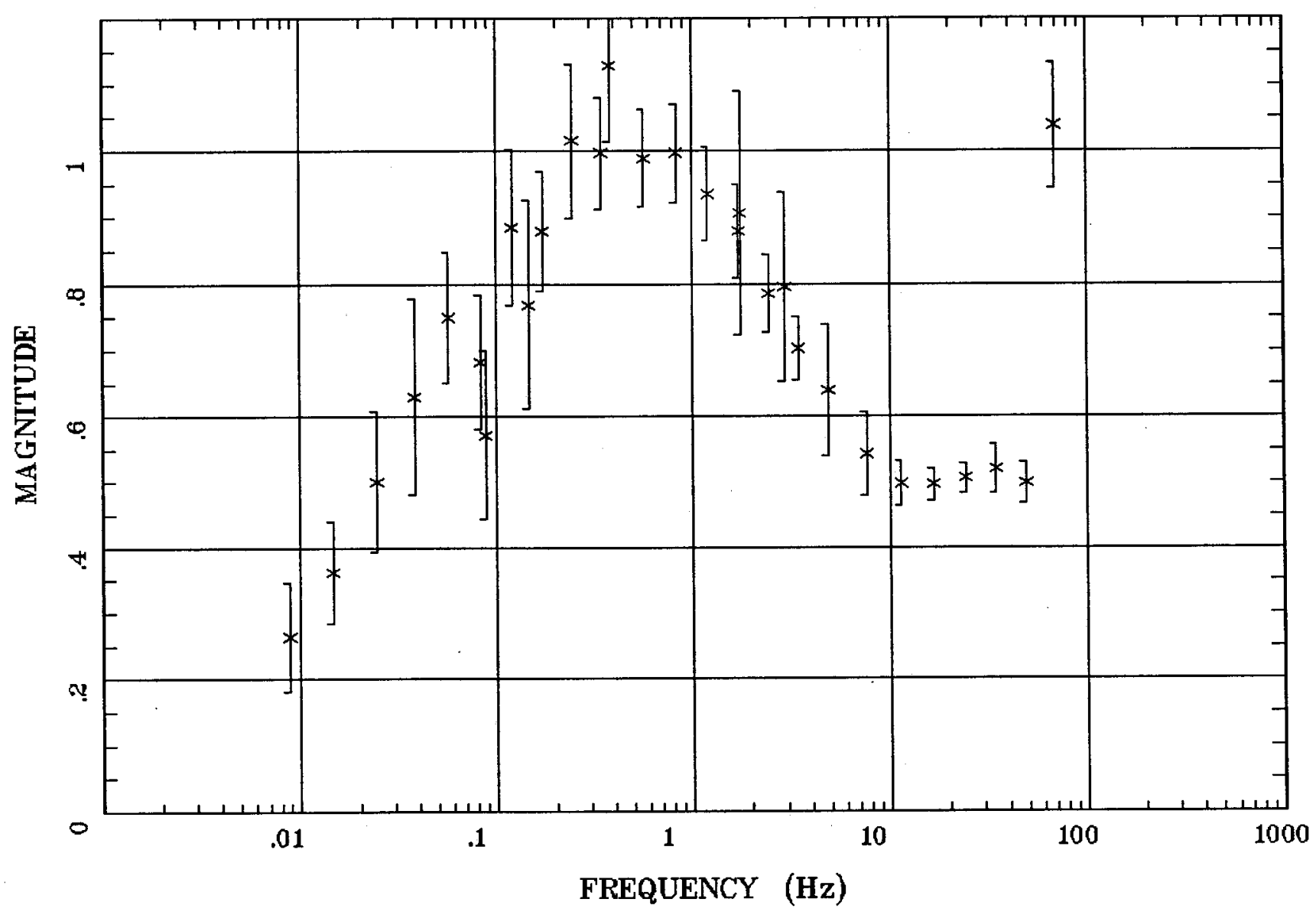

Client:

Remote: none

Acquired: 16:3 Jul 28, 2002 Survey Co:USGS
Rotation:

Filename: eb03all.avg

Channels: Ch1 Ch2 Ch3 Ch4 Ch5 Ch3 Ch4 Plotted: 09:55 Jan 11, 2006

< EMI - ElectroMagnetic Instruments 


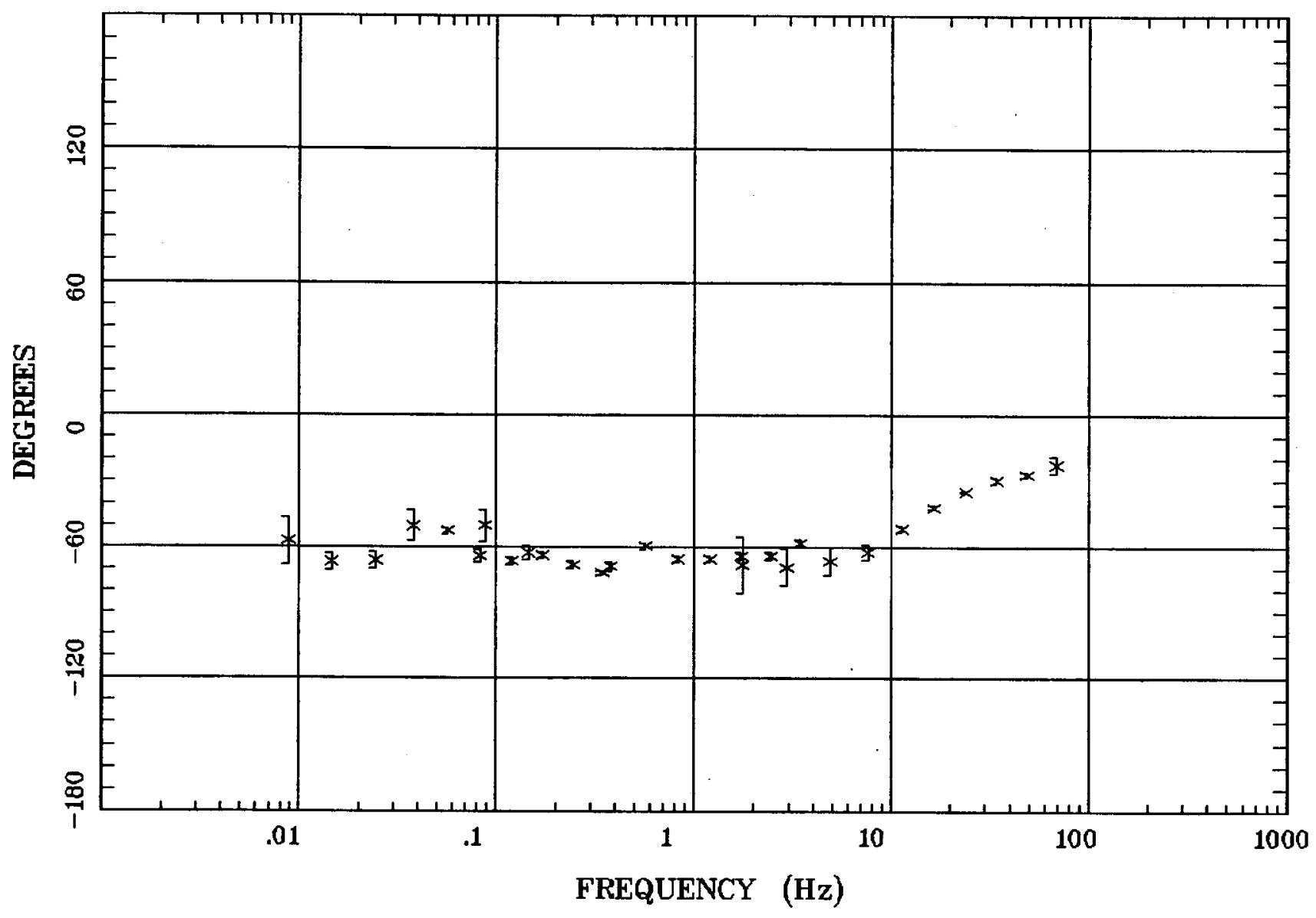

Client:

Remote: none

Acquired: $16: 3$ Jul 28, 2002 Survey Co:USGS
Rotation:

Filename: eb03all.avg

Channels: Ch1 Ch2 Ch3 Ch4 Ch5 Ch3 Ch4

Plotted: 09:55 Jan 11, 2006

< EMI - ElectroMagnetic Instruments 
HzHx.x Coh HzHy.o

Espanola, NM

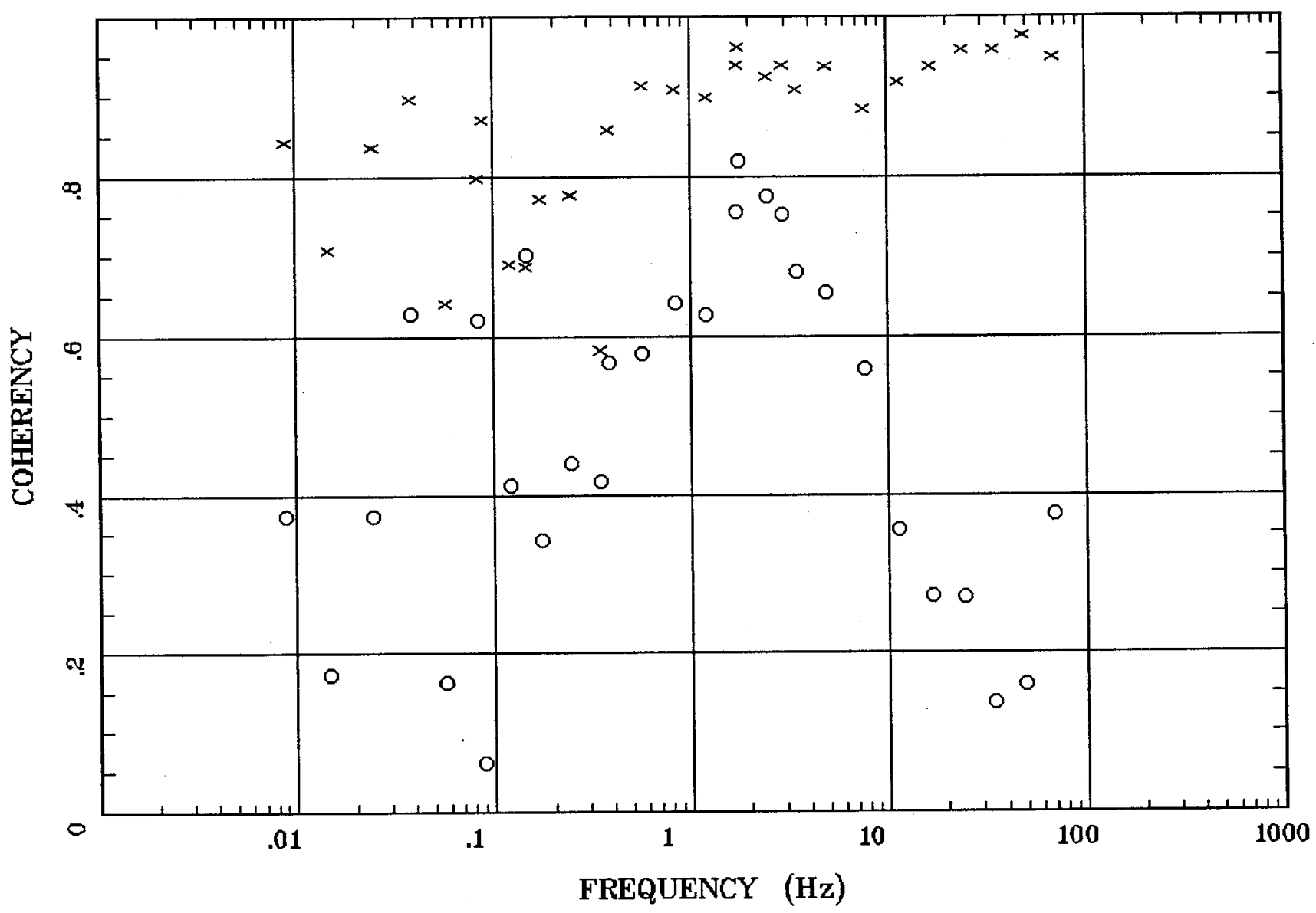

Client:

Remote: none

Acquired: 16:3 Jul 28, 2002 Survey Co:USGS
Rotation:

Filename: eb03all.avg

Channels: Ch1 Ch2 Ch3 Ch4 Ch5 Ch3 Ch4 Plotted: 09:55 Jan 11, 2006

$<$ EMI - ElectroMagnetic Instruments 


\section{Station RR01}

APPARENT RESISTIVITY

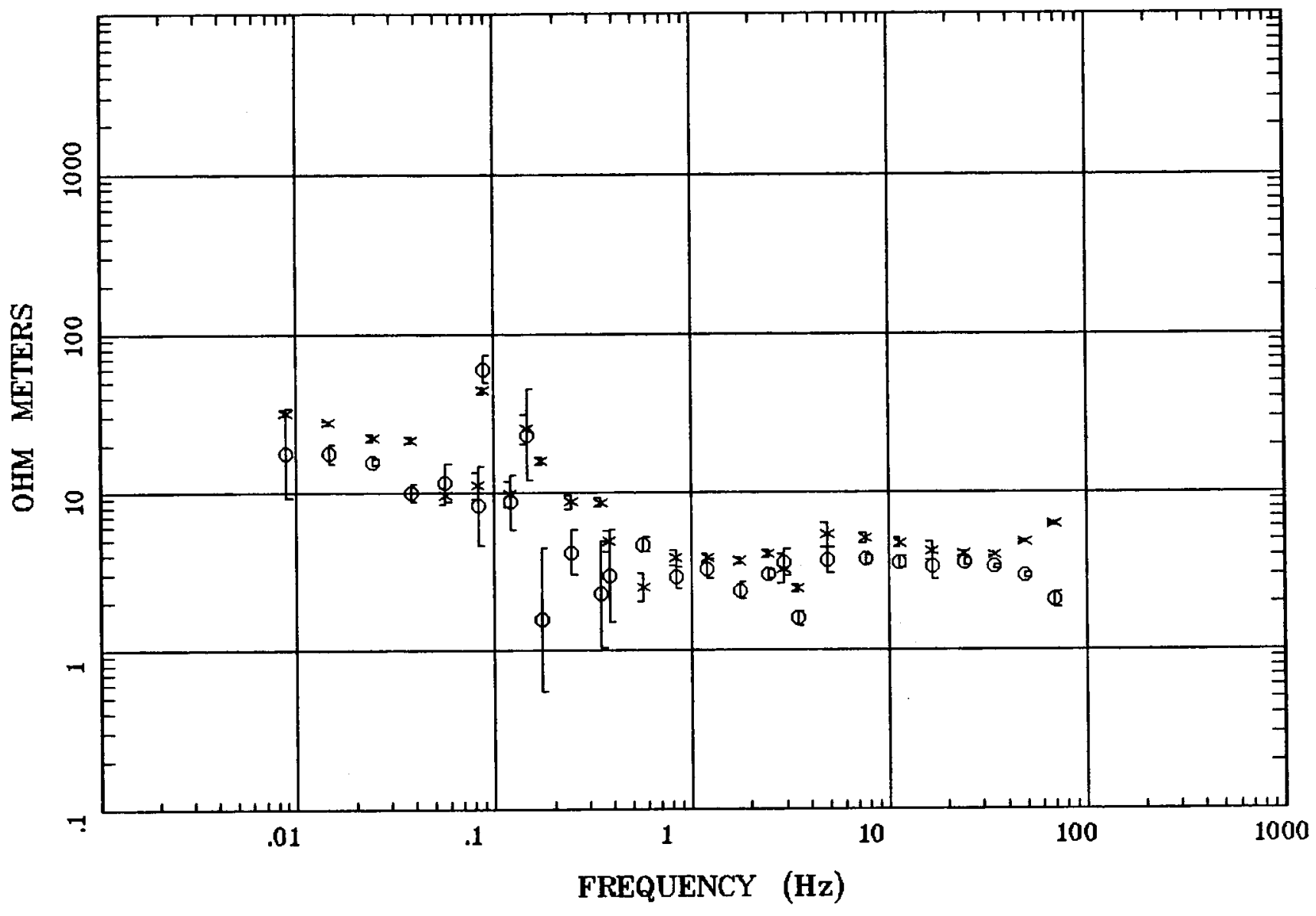

Client: Espanola Basin

Remote: none

Acquired: 10:4 Jul 20, 2004

Survey Co:USGS
Rotation:

Filename: rrlm56.avg

Plotted: 11:13 Sep 24, 2004

$<$ EMI - ElectroMagnetic Instruments
Channels: Ch1 Ch2 Ch3 Ch4 Ch5 Ch3 Ch4 


\section{Station RR01}

IMPEDANCE PHASE

Albuquerque NM 1:100K

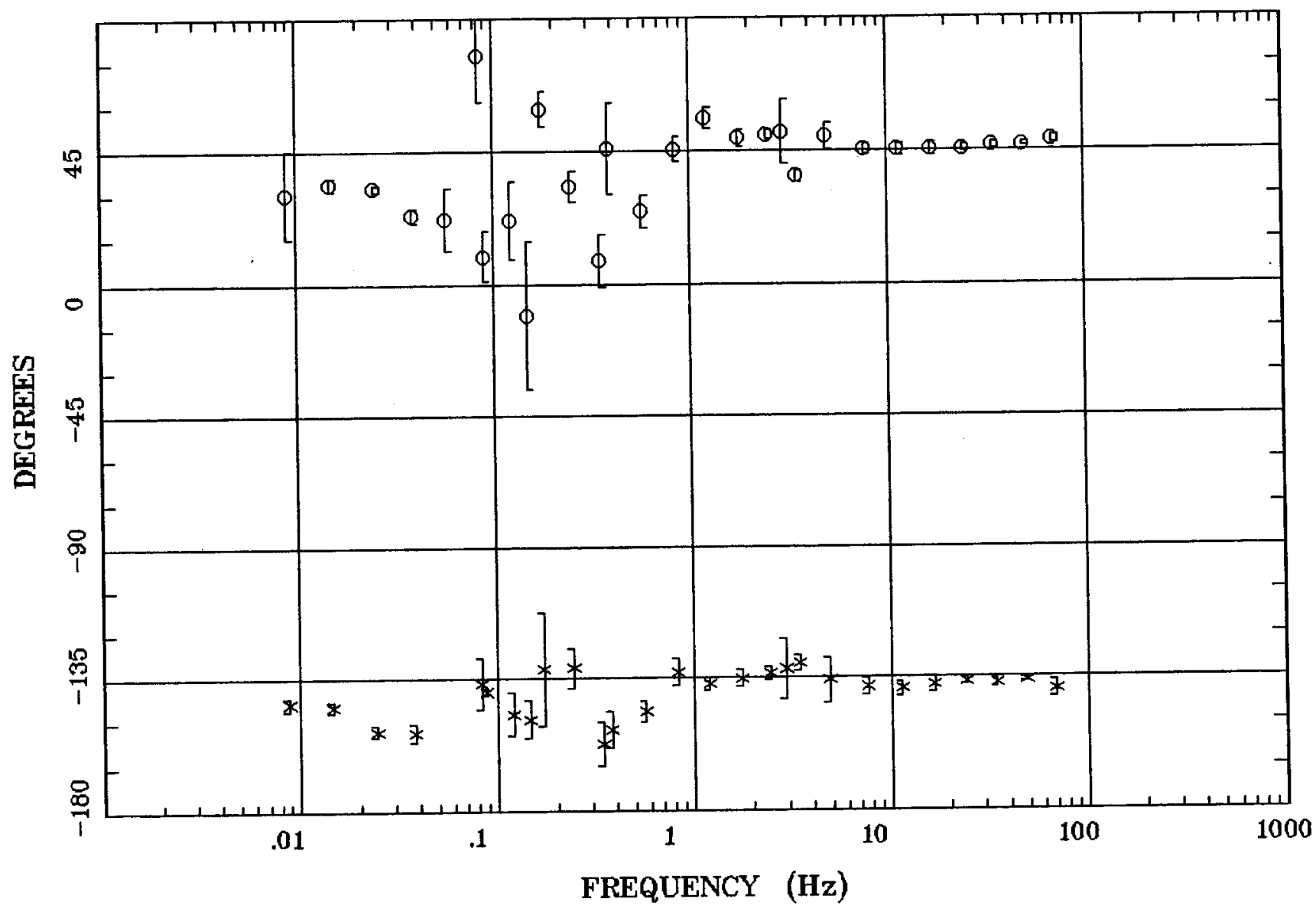

Client: Espanola Basin

Remote: none

Acquired: 10:4 Jul 20, 2004

Survey Co:USGS
Rotation:

Filename: rrim56.avg

Channels: Ch1 Ch2 Ch3 Ch4 Ch5 Ch3 Ch4

Plotted: 11:13 Sep 24, 2004

< EMI - ElectroMagnetic Instruments > 


\section{Station RR01}

ROTATION ANGLE

Albuquerque NM 1:100K

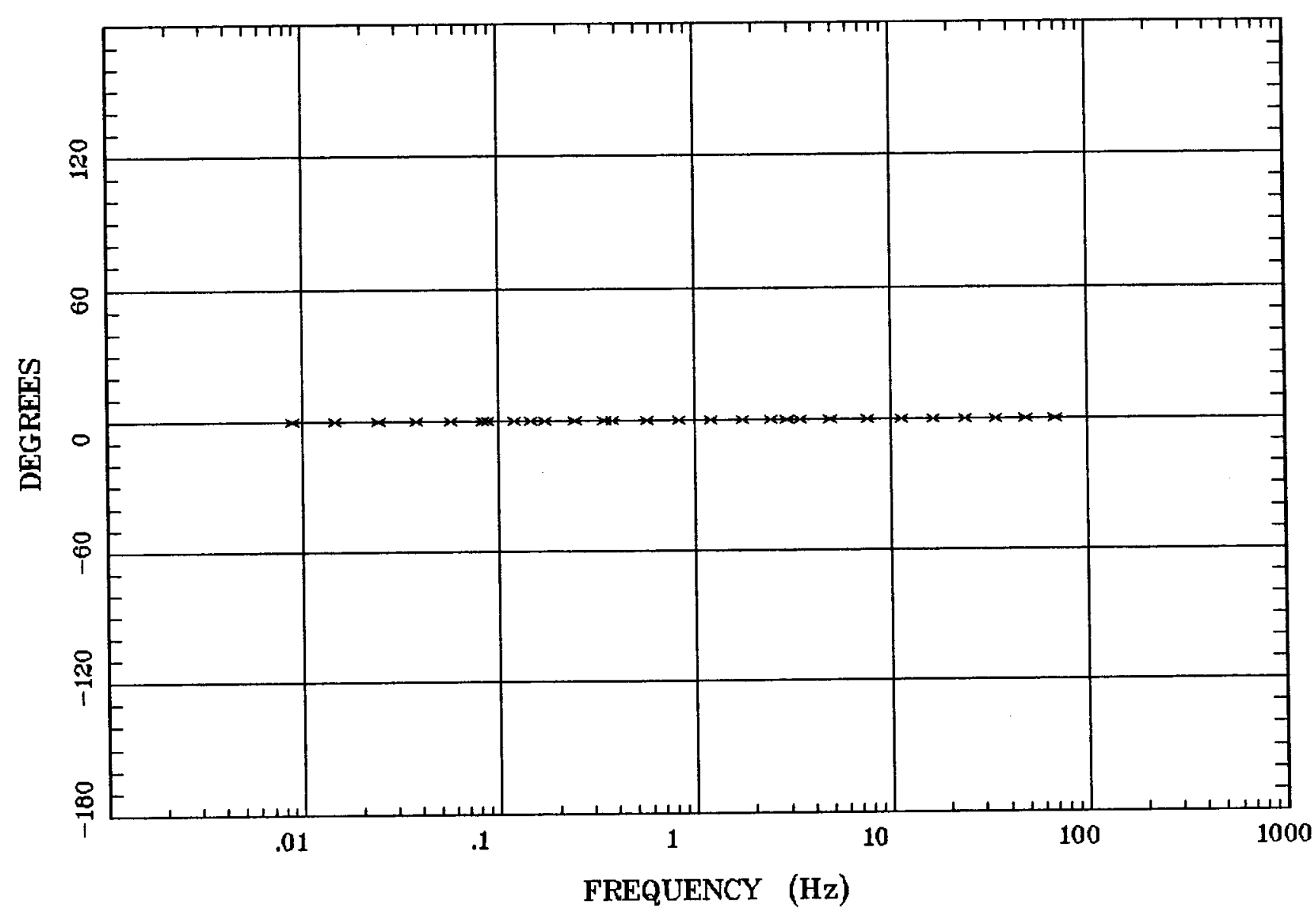

Client: Espanola Basin

Remate: none

Acquired: 10:4 Jul 20, 2004

Survey Co:USGS
Rotation:

Filename: rrim56.avg

Channels: Ch1 Ch2 Ch3 Ch4 Ch5 Ch3 Ch4 Plotted: 11:13 Sep 24, 2004

$<$ EMI - ElectroMagnetic Instruments 
Station RR01

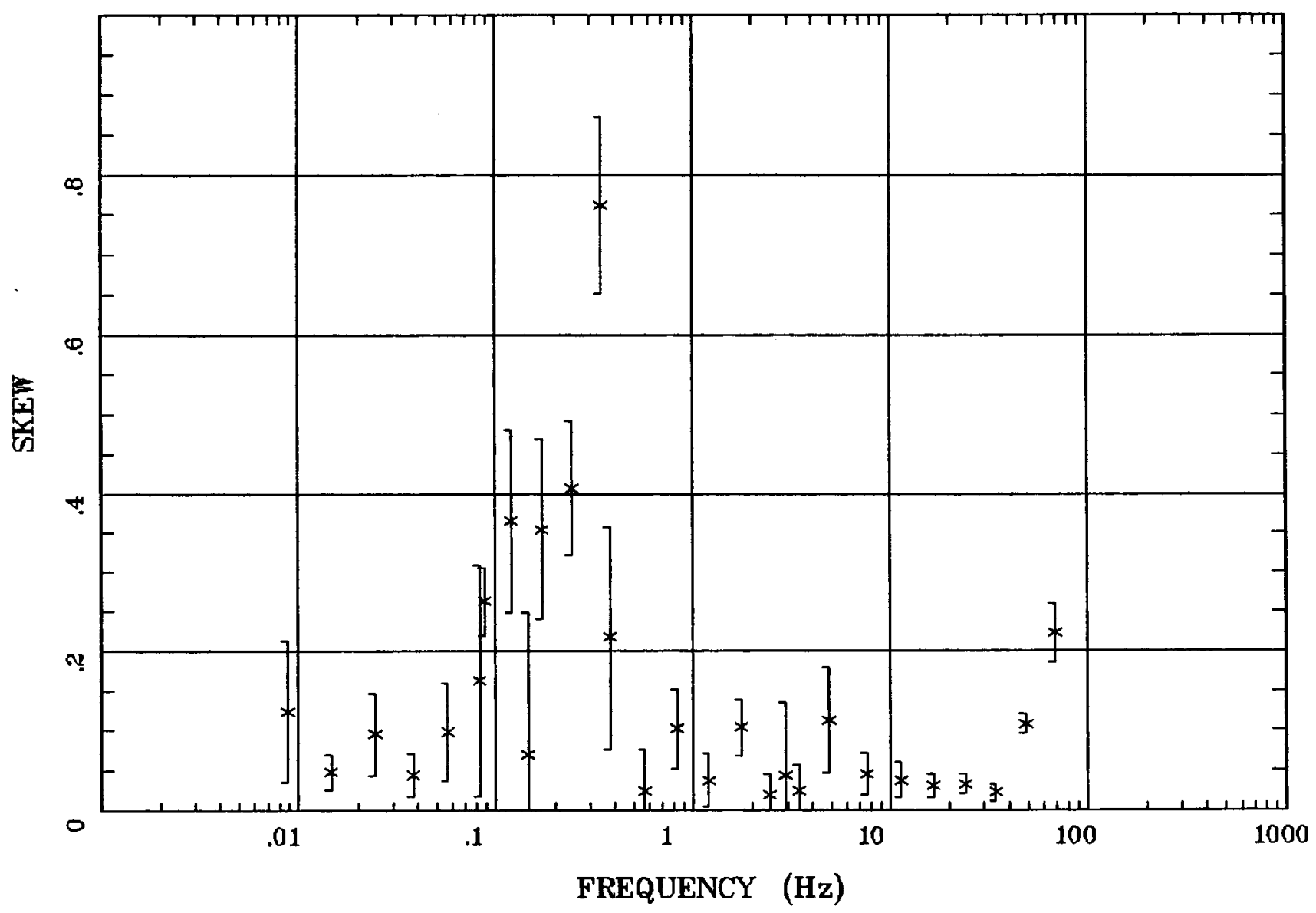

Client: Espanola Basin

Remote: none

Acquired: 10:4 Jul 20, 2004

Survey Co:USGS
Rotation:

Filename: rrim56.avg

Channels: Ch1 Ch2 Ch3 Ch4 Ch5 Ch3 Ch4 Plotted: 11:13 Sep 24, 2004

< EMI - ElectroMagnetic Instruments > 


\section{Station RR01}

E MULT Coh.

Albuquerque NM 1:100K

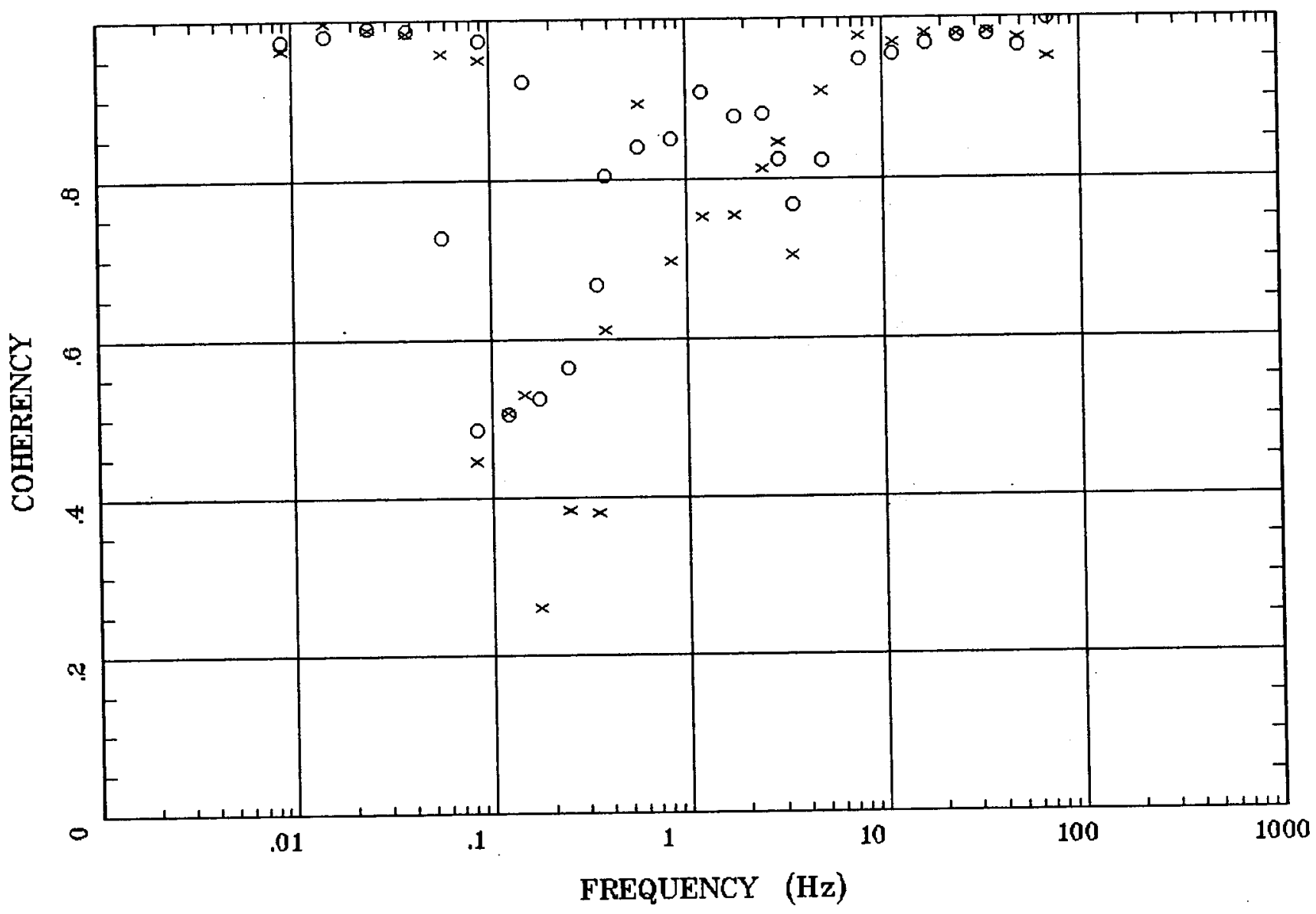

Client: Espanola Basin Remote: none Acquired: 10:4 Jul 20, 2004 Survey Co:USGS
Rotation:

Filename: rr1m56.avg

Channels: Ch1 Ch2 Ch3 Ch4 Ch5 Ch3 Ch4 Plotted: 11:13 Sep 24, 2004

< EMI - ElectroMagnetic Instruments > 


\section{Station RR01}

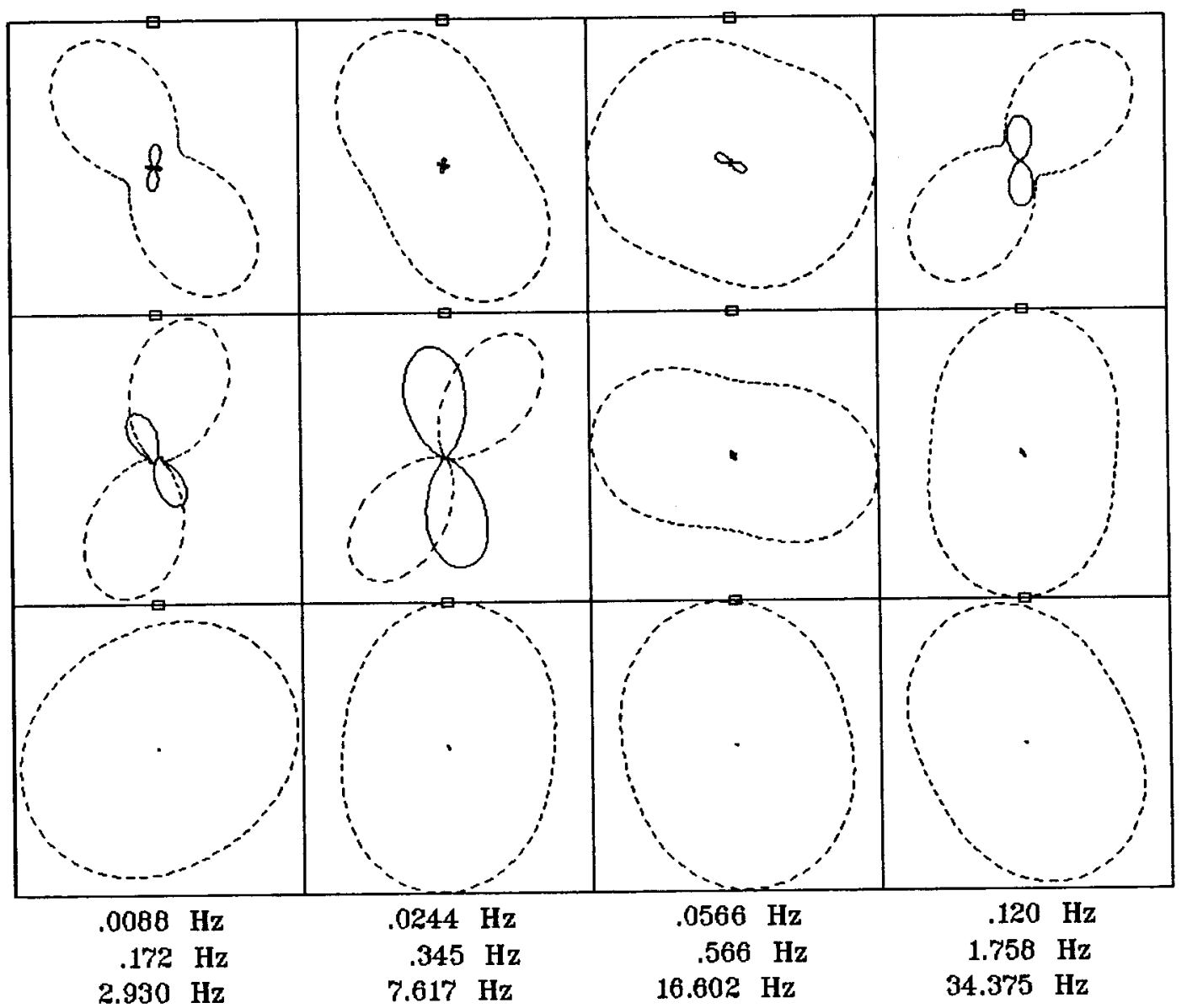

Client: Espanola Basin

Remote: none

Acquired: 10:4 Jul 20, 2004

Survey Co:USGS
Rotation:

Filename: rrim56.avg

Channels: Ch1 Ch2 Ch3 Ch4 Ch5 Ch3 Ch4 Plotted: 11:13 Sep 24, 2004

< EMI - ElectroMagnetic Instruments > 


\section{Station RR01}

TIPPER MAGNITUDE

Albuquerque NM 1:100K

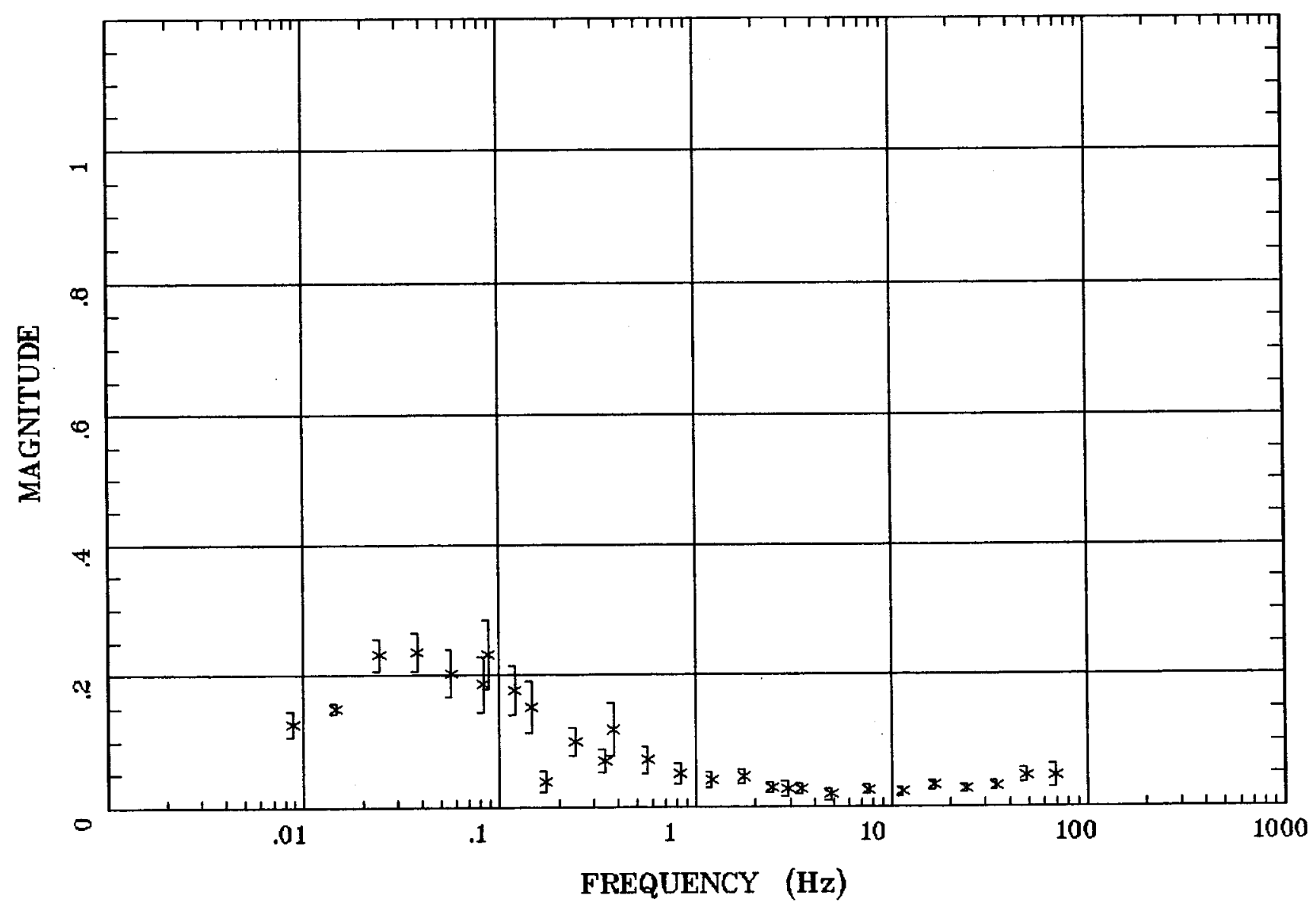

Client: Espanola Basin Remote: none Acquired: 10:4 Jul 20, 2004 Survey Co:USGS
Rotation:

Filename: rrim56.avg

Channels: Ch1 Ch2 Ch3 Ch4 Ch5 Ch3 Ch4 Plotted: 11:13 Sep 24, 2004

< EMI - ElectroMagnetic Instruments > 


\section{Station RR01}

TIPPER STRIKE

Albuquerque NM 1:100K



Client: Espanola Basin Remote: none

Acquired: 10:4 Jul 20, 2004 Survey Co:USGS

\section{Rotation:}

Filename: rr1m56.avg

Channels: Ch1 Ch2 Ch3 Ch4 Ch5 Ch3 Ch4 Plotted: 11:13 Sep 24, 2004

< EMI - ElectroMagnetic Instruments > 


\section{Station RR01}

HzHx.x Coh HzHy.o

Albuquerque NM 1:100K

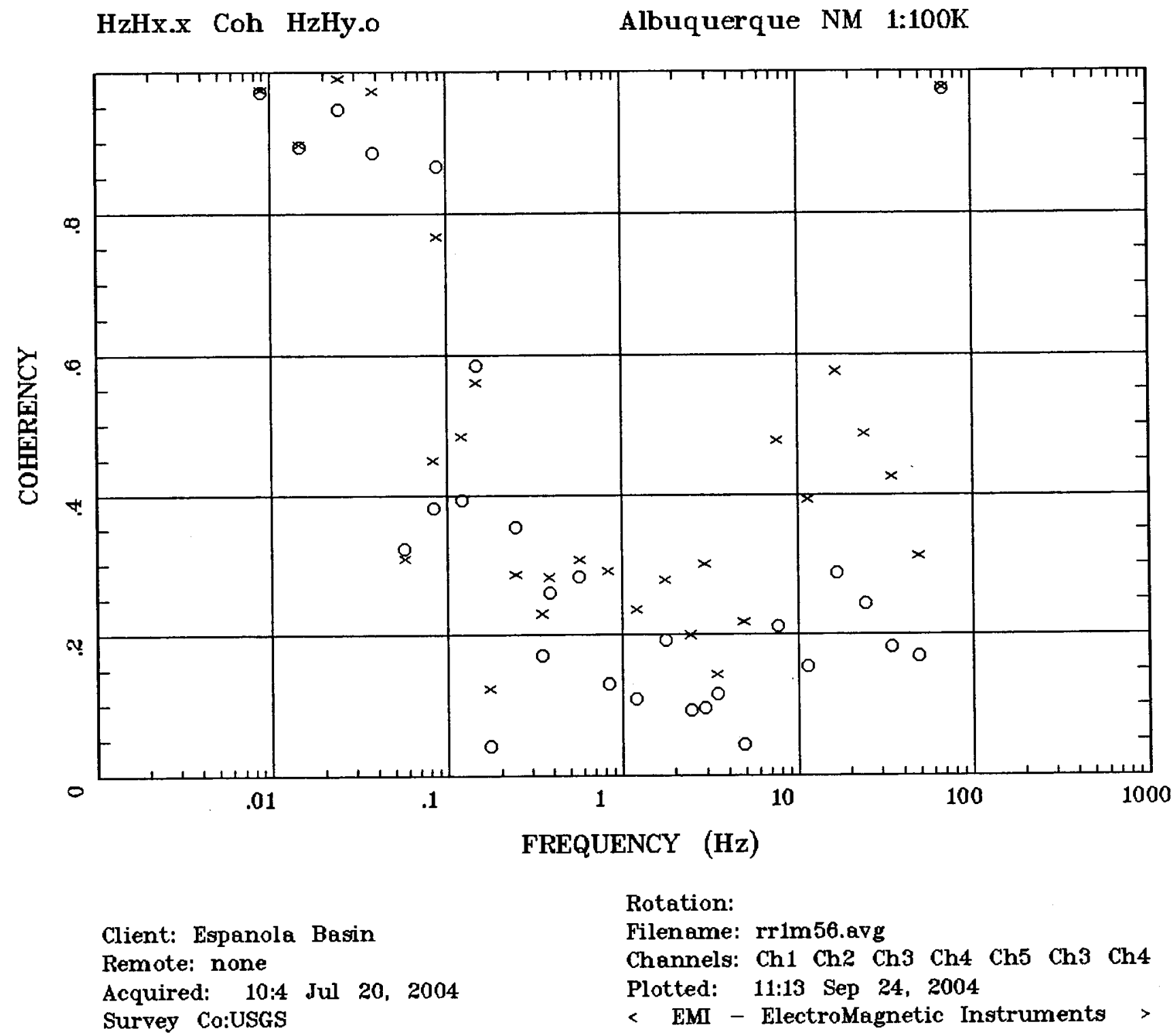


APPARENT RESISTIVITY

Albuquerque NM 1:100K

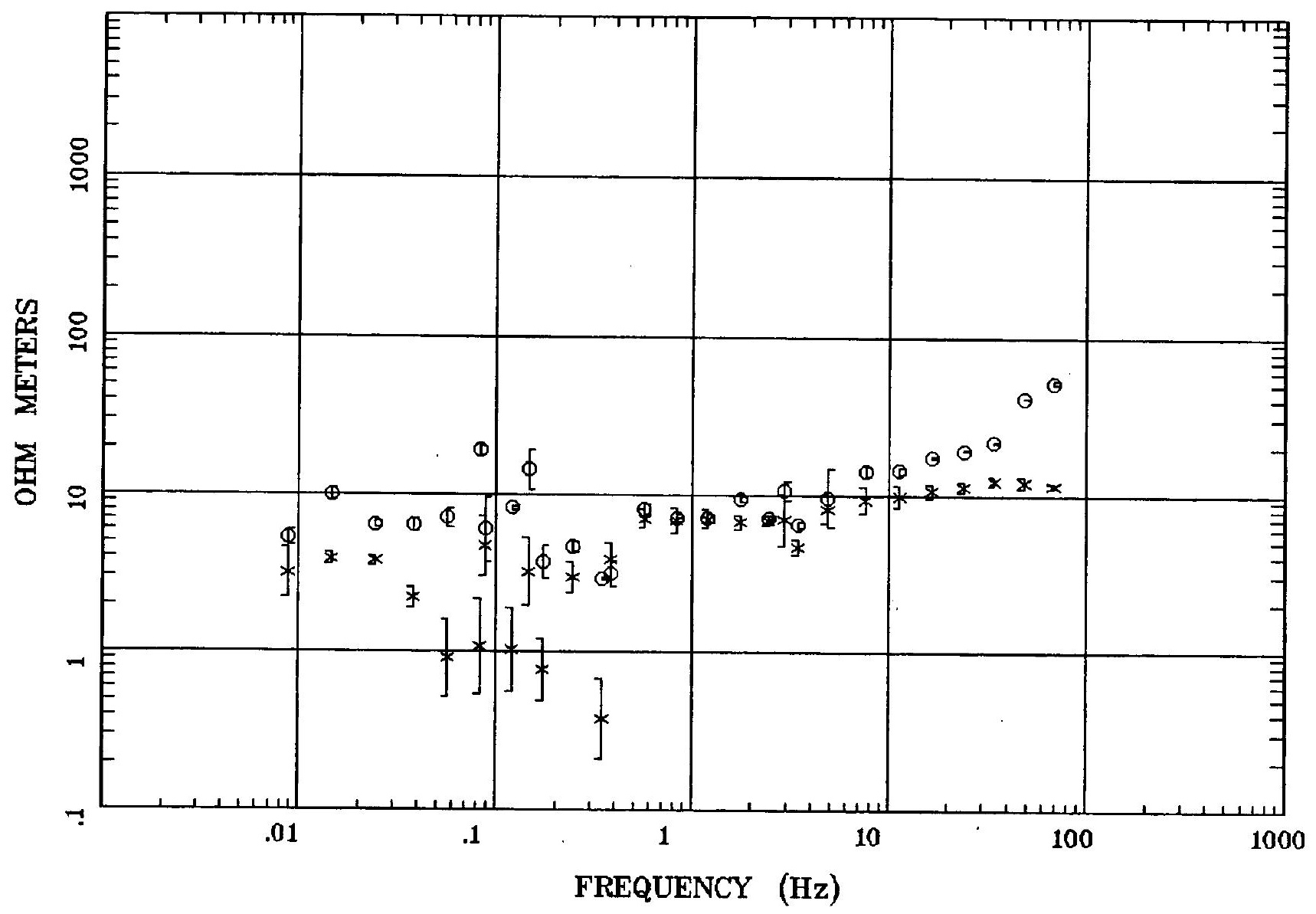

Client: Espanala Basin Remote: none Acquired: 14:3 Jul 20, 2004 Survey Co:USGS
Rotation:

Filename: rr2m63.avg

Channels: Ch1 Ch2 Ch3 Ch4 Ch5 Ch3 Ch4

Plotted: 11:23 Sep 24, 2004

< EMI - ElectroMagnetic Instruments > 


\section{Station RR02}

IMPEDANCE PHASE

Albuquerque NM 1:100K

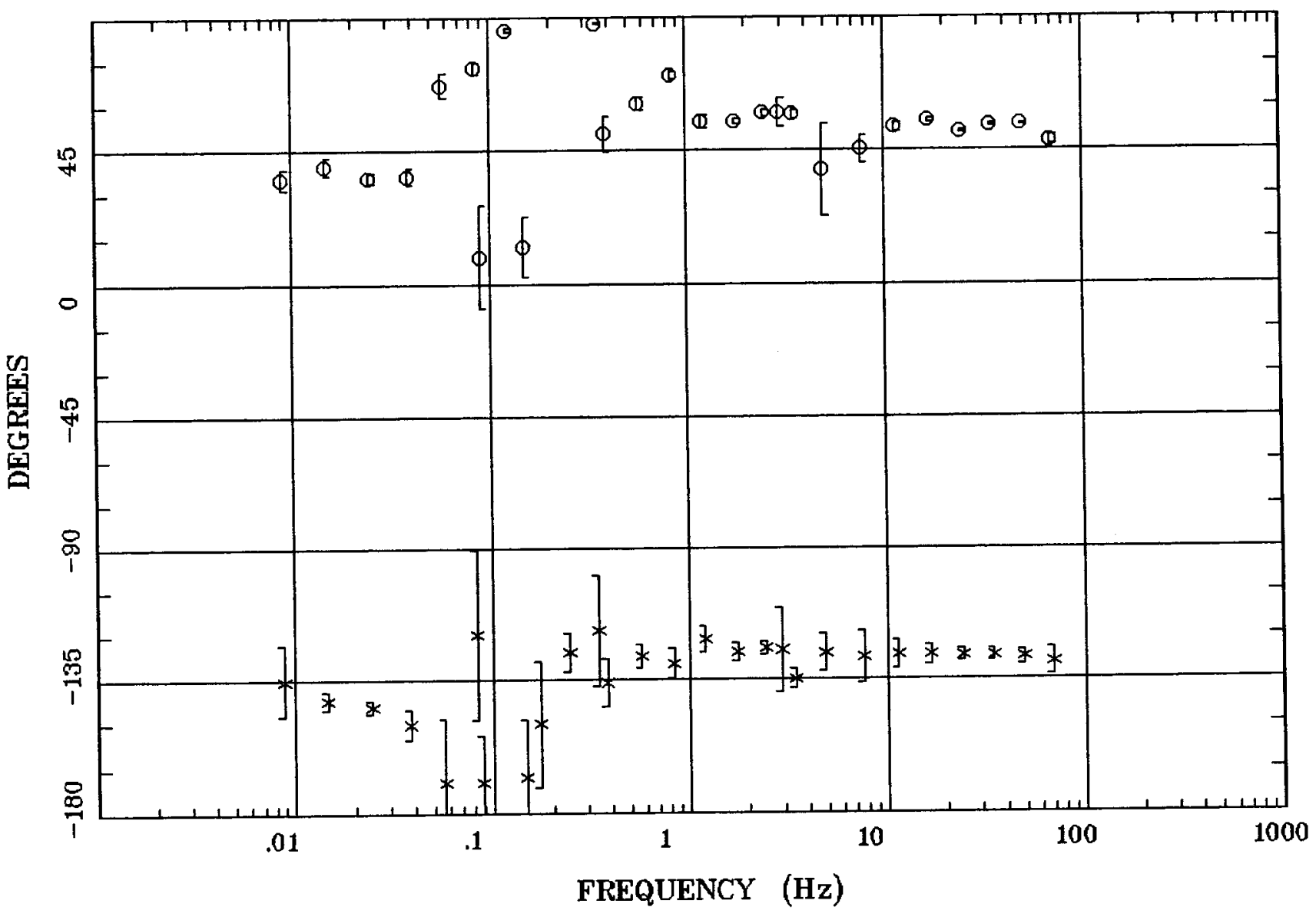

Client: Espanola Basin

Rotation:

Remote: none

Filename: rr2m63.avg

Channels: Ch1 Ch2 Ch3 Ch4 Ch5 Ch3 Ch4

Acquired: 14:3 Jul 20, 2004

Survey Co:USGS

Plotted: 11:23 Sep 24, 2004

< EMI - ElectroMagnetic Instruments > 


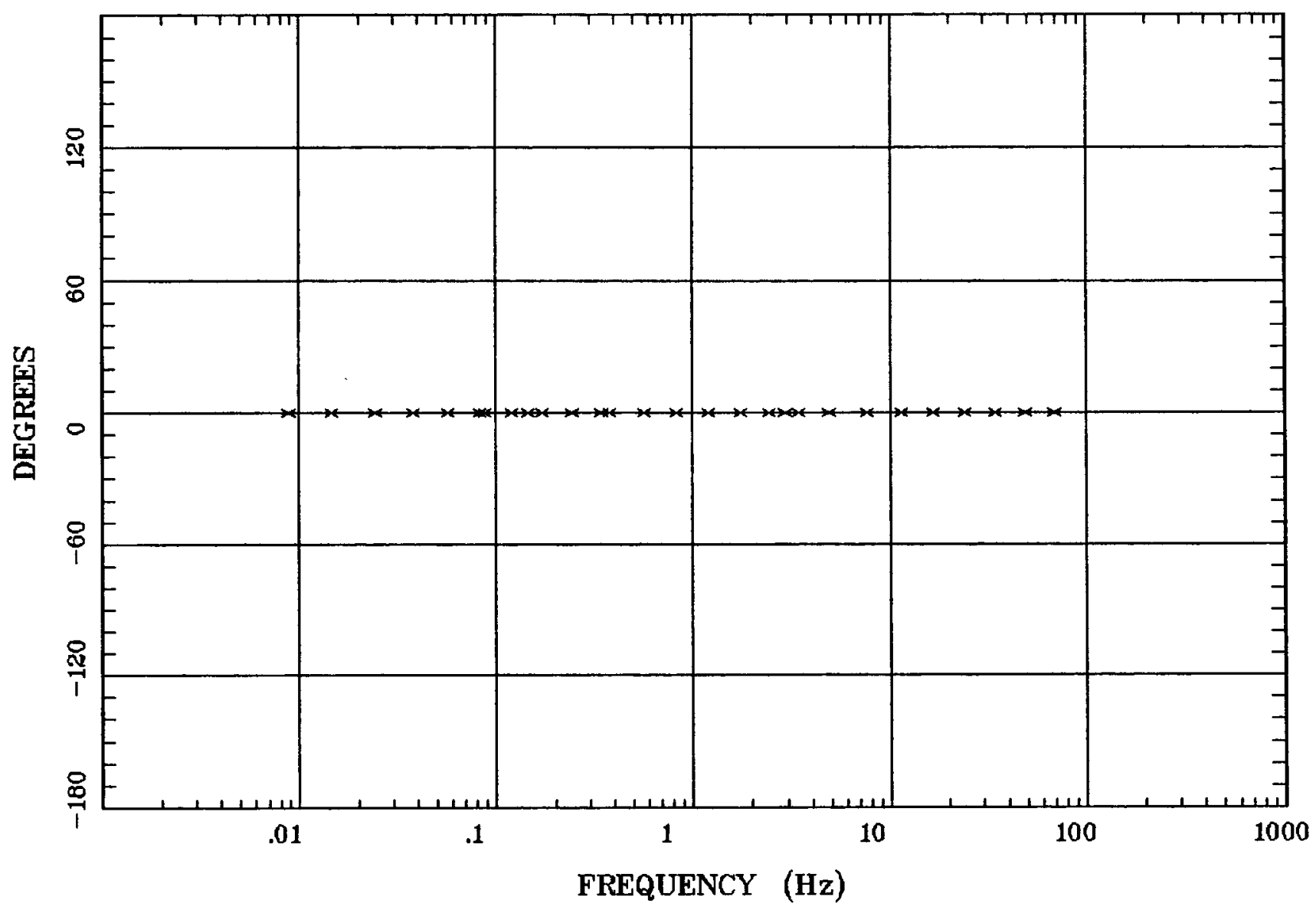

Client: Espanola Basin

Remote: none

Acquired: 14:3 Jul 20, 2004

Survey Co:USGS
Rotation:

Filename: rrzm63.avg

Channels: Ch1 Ch2 Ch3 Ch4 Ch5 Ch3 Ch4

Plotted: 11:23 Sep 24, 2004

< EMI - ElectroMagnetic Instruments > 


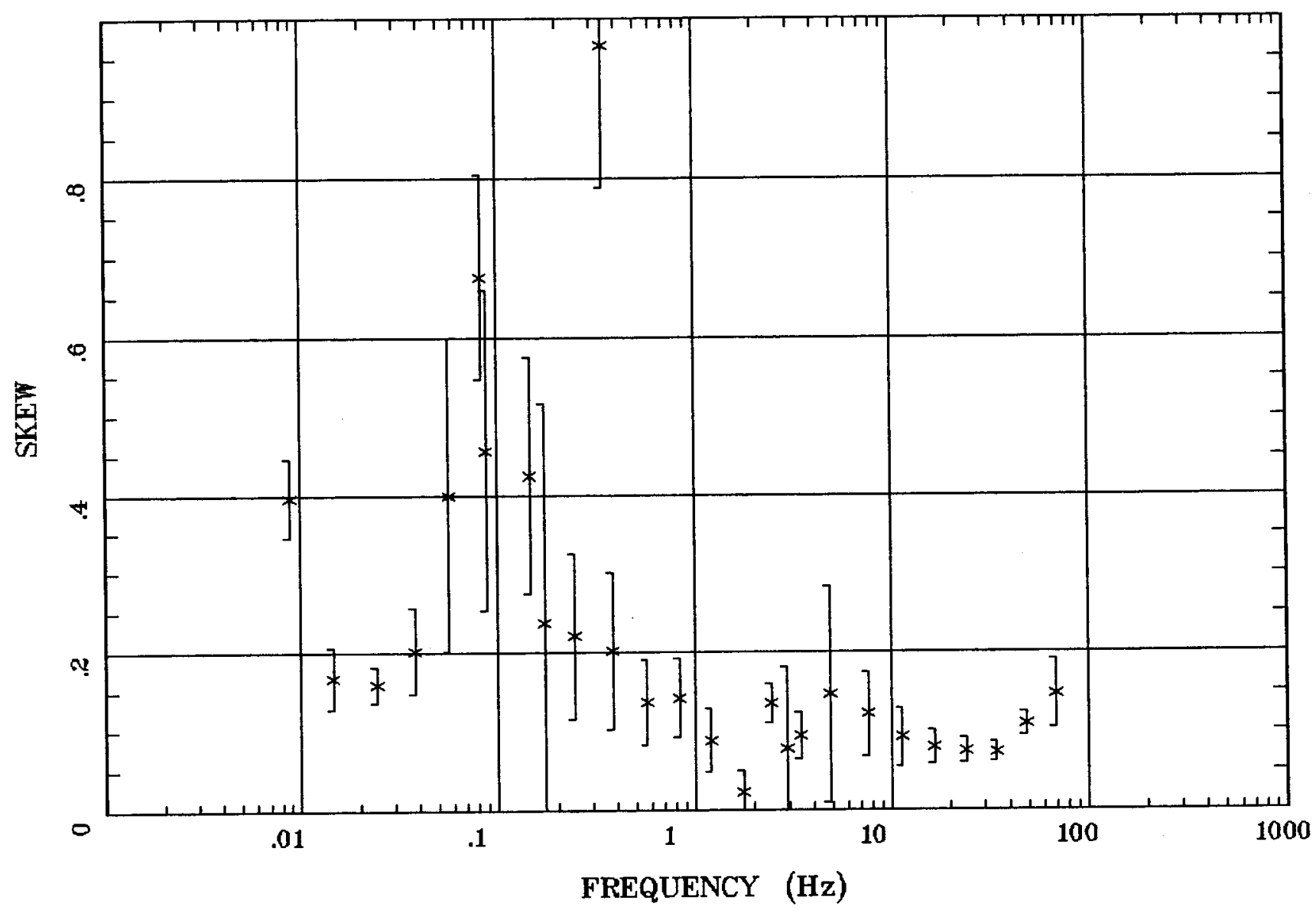

Client: Espanola Basin Remote: none Acquired: 14:3 Jul 20, 2004 Survey Co:USGS
Rotation:

Filename: rr2m63.avg

Channels: Ch1 Ch2 Ch3 Ch4 Ch5 Ch3 Ch4 Plotted: 11:23 Sep 24, 2004

< EMI - ElectroMagnetic Instruments > 


\section{Station RR02}

E MULT Coh.

Albuquerque NM 1:100K

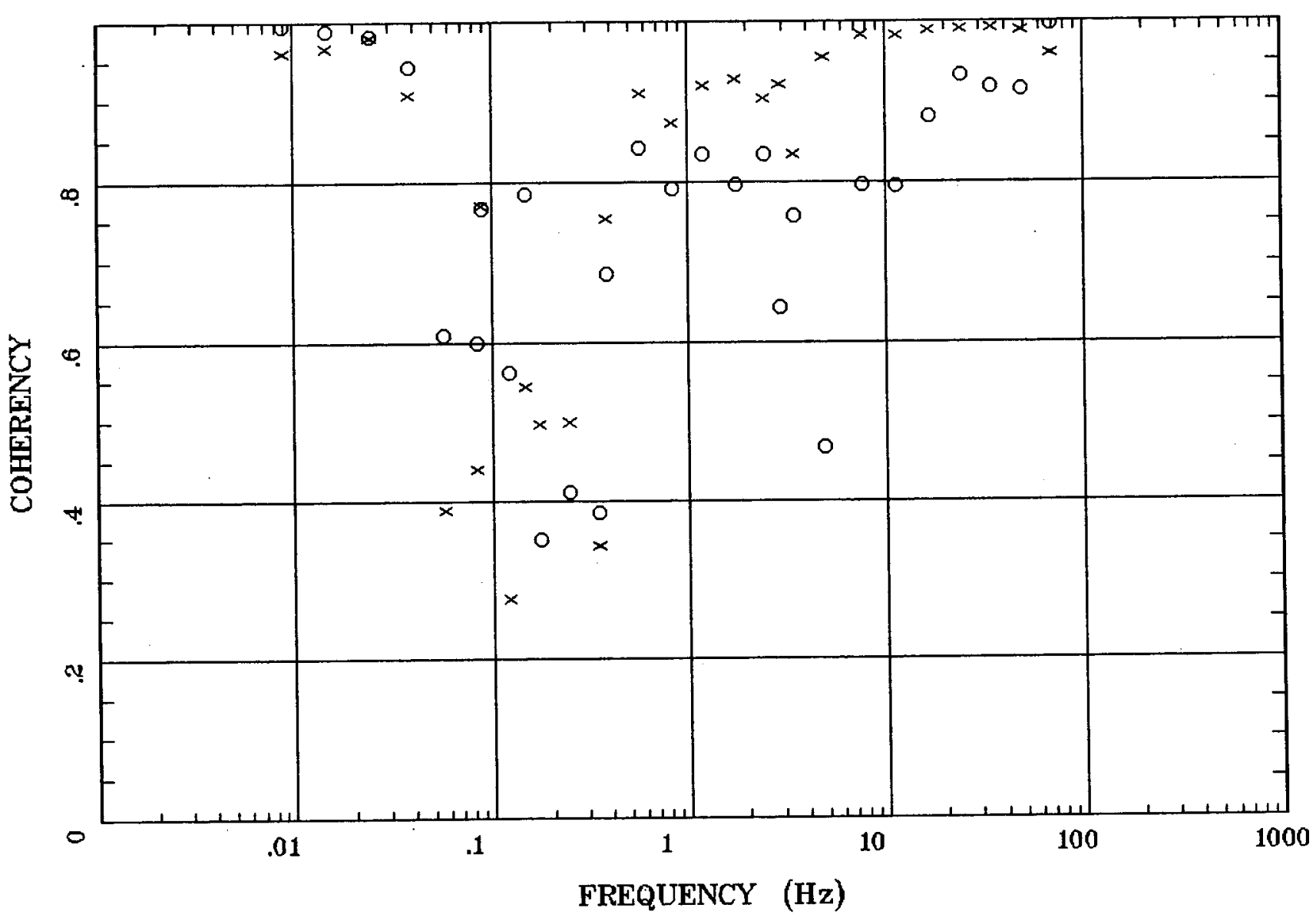

Client: Espanola Basin

Remote: none

Acquired: 14:3 Jul 20, 2004

Survey Co:USGS
Rotation:

Filename: rr2m63.avg

Channels: Ch1 Ch2 Ch3 Ch4 Ch5 Ch3 Ch4

Platted: 11:23 Sep 24, 2004

$<$ EMI - ElectroMagnetic Instruments 


\section{POLAR PLOTS \\ Albuquerque NM 1:100K}

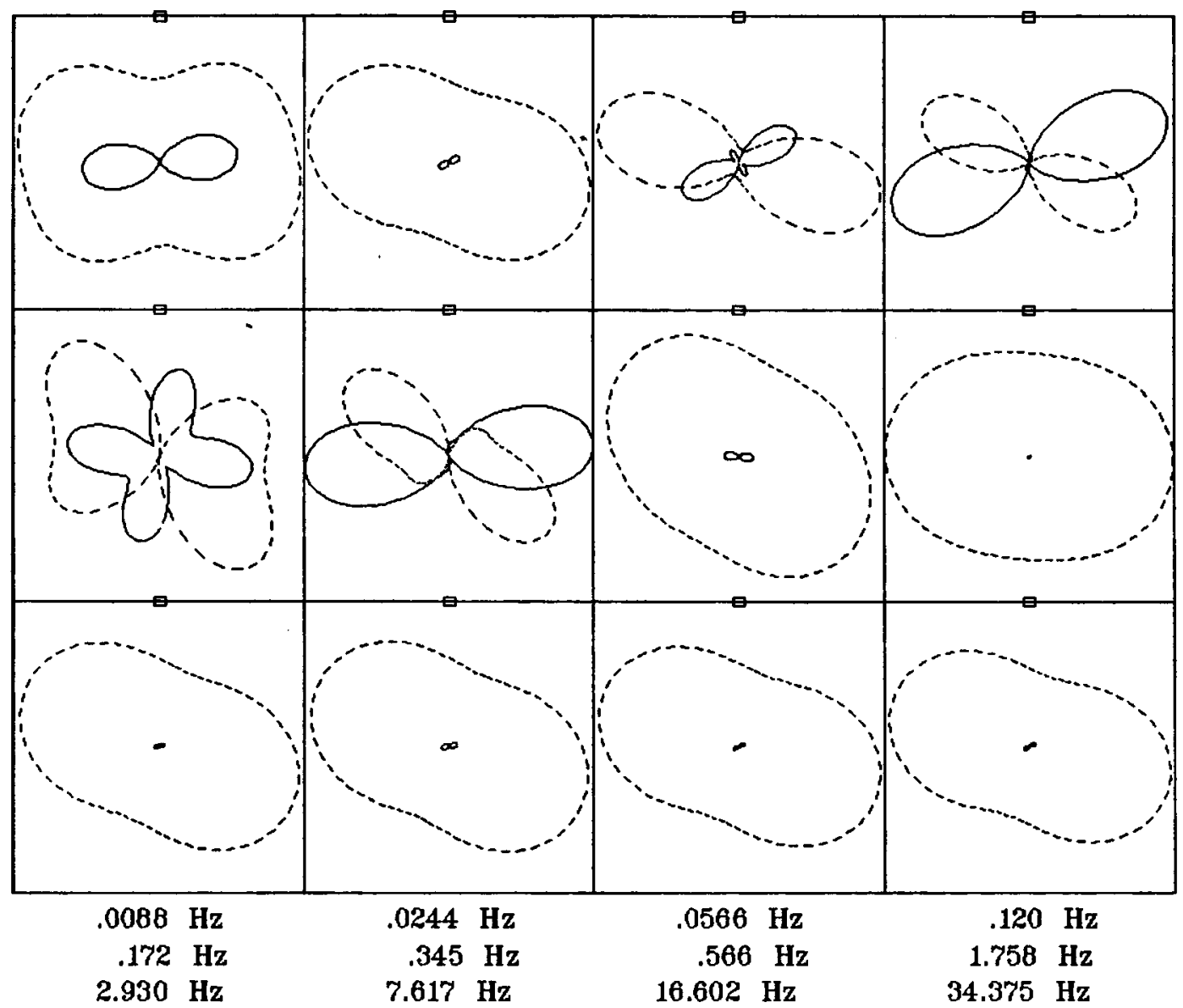

Client: Espanola Basin

Remote: none

Acquired: 14:3 Jul 20, 2004

Survey Co:USGS
Rotation:

Filename: rr2m63.avg

Channels: Ch1 Ch2 Ch3 Ch4 Ch5 Ch3 Ch4

Plotted: 11:23 Sep 24, 2004

< EMI - ElectroMagnetic Instruments 


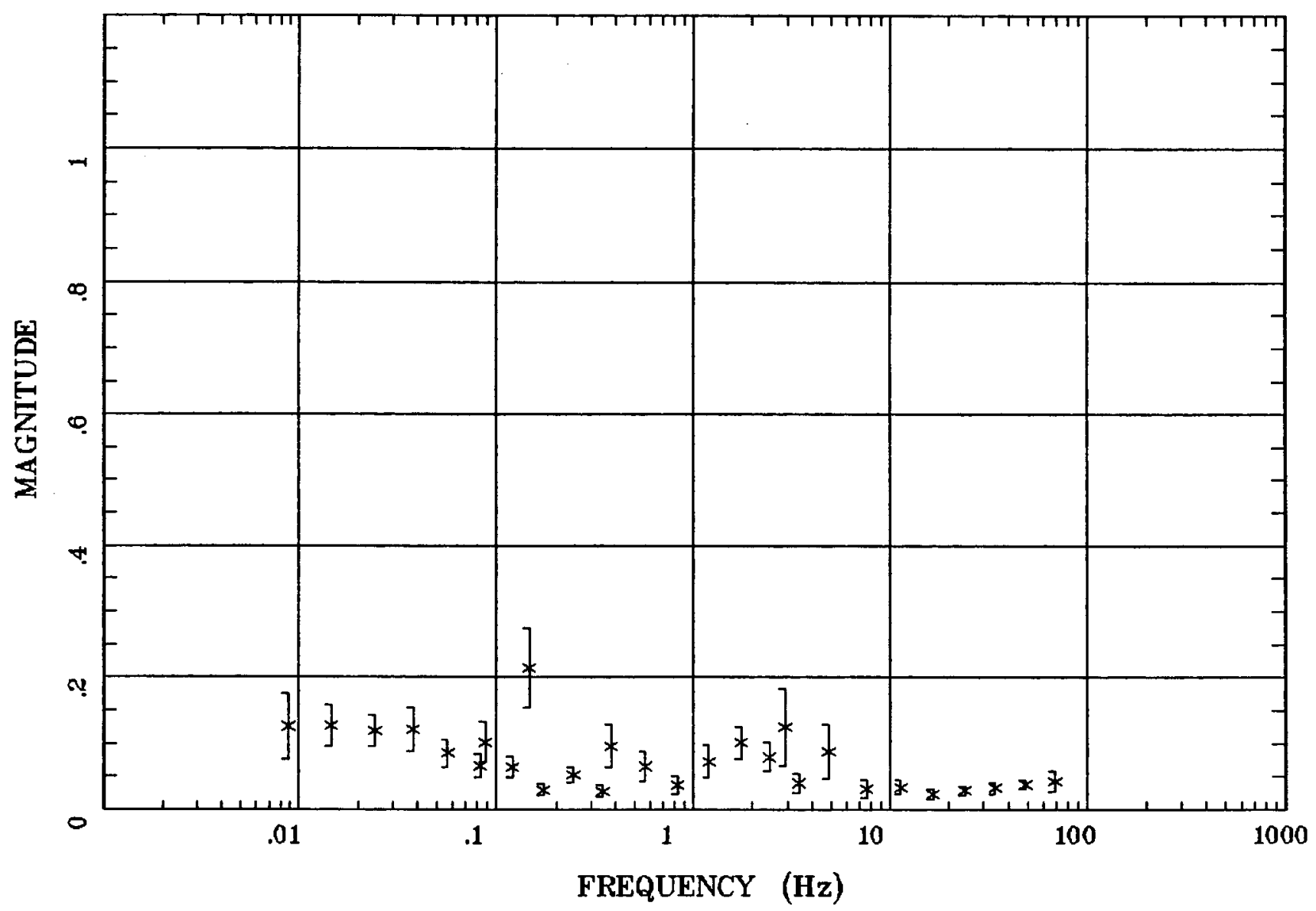

Client: Espanola Basin Remote: none Acquired: 14:3 Jul 20, 2004 Survey co:USGS

\section{Rotation:}

Filename: rr2m63.avg

Channels: Ch1 Ch2 Ch3 Ch4 Ch5 Ch3 Ch4 Plotted: 11:23 Sep 24, 2004

< EMI - ElectroMagnetic Instruments > 


\section{Station RR02}

TIPPER STRIKE

Albuquerque NM 1:100K



Client: Espanola Basin

Remote: none

Acquired: 14:3 Jul 20, 2004

Survey Co:USGS
Rotation:

Filename: rr2m63.avg

Channels: Ch1 Ch2 Ch3 Ch4 Ch5 Ch3 Ch4 Plotted: 11:23 Sep 24, 2004

< EMI - ElectroMagnetic Instruments > 


\section{Station RR02}

HzHx.x Coh HzHy.o

Albuquerque NM 1:100K

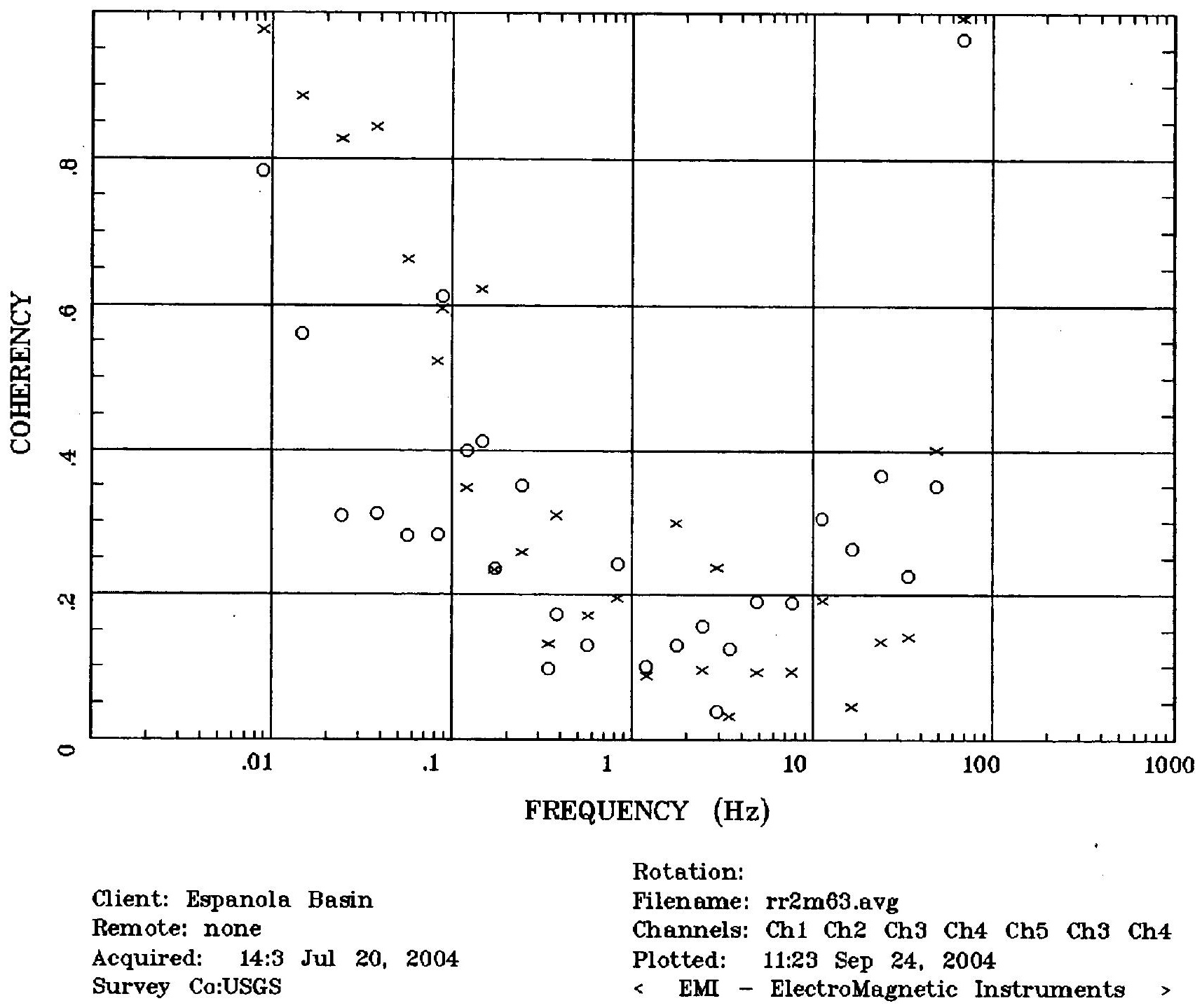




\section{Station RR20}

\section{APPARENT RESISTIVITY}

Albuquerque NM 1:100K

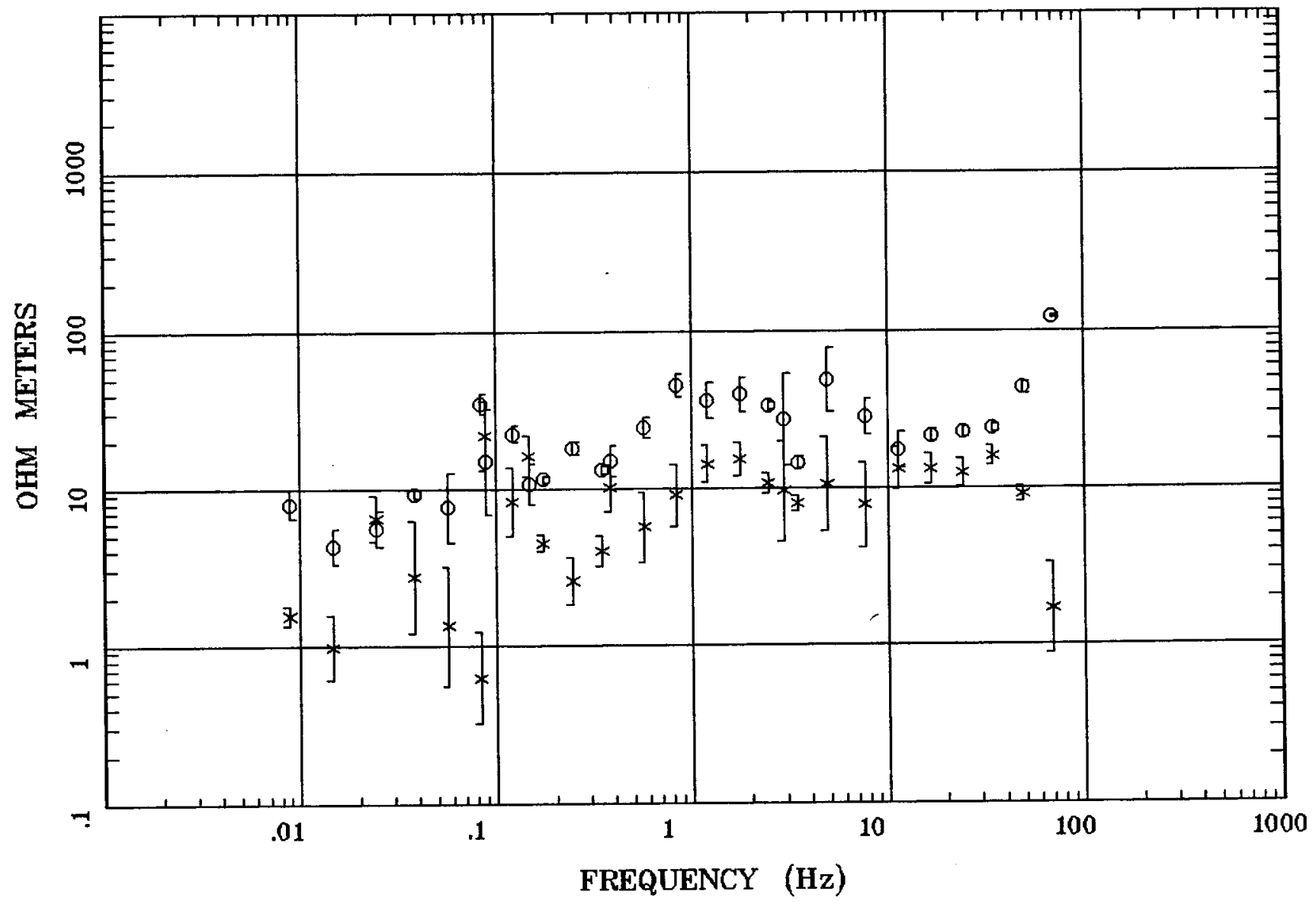

Client: Espanola Basin

Remote: none

Acquired: 11:3 Jul 17, 2004

Survey Co:USGS
Rotation:

Filename: rr20m25.avg

Channels: Ch1 Ch2 Ch3 Ch4 Ch5 Ch3 Ch4

Plotted: 15:31 Sep 01, 2004

< EMI - ElectroMagnetic Instruments > 




Client: Espanola Basin Remote: none Acquired: 11:3 Jul 17, 2004 Survey Co:USGS
Rotation:

Filename: rr20m25.avg

Channels: Ch1 Ch2 Ch3 Ch4 Ch5 Ch3 Ch4 Plotted: 15:31 Sep 01, 2004

$<$ EMI - ElectroMagnetic Instruments > 


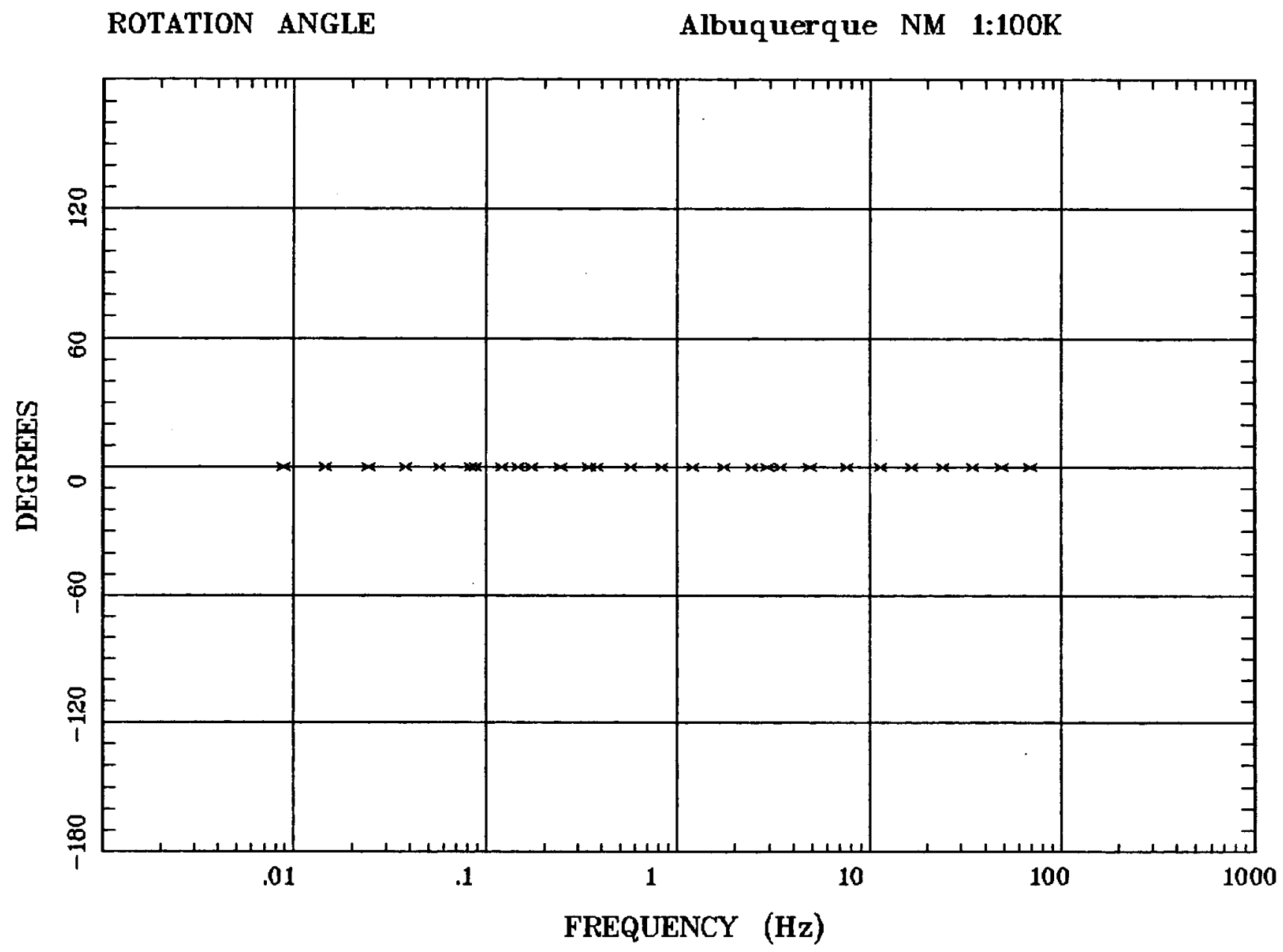

Client: Espanola Basin

Remote: none

Acquired: 11:3 Jul 17, 2004

Survey Co:USGS
Rotation:

Filename: rr20m25.avg

Channels: Ch1 Ch2 Ch3 Ch4 Ch5 Ch3 Ch4

Plotted: 15:31 Sep 01, 2004

< EMI - ElectroMagnetic Instruments 


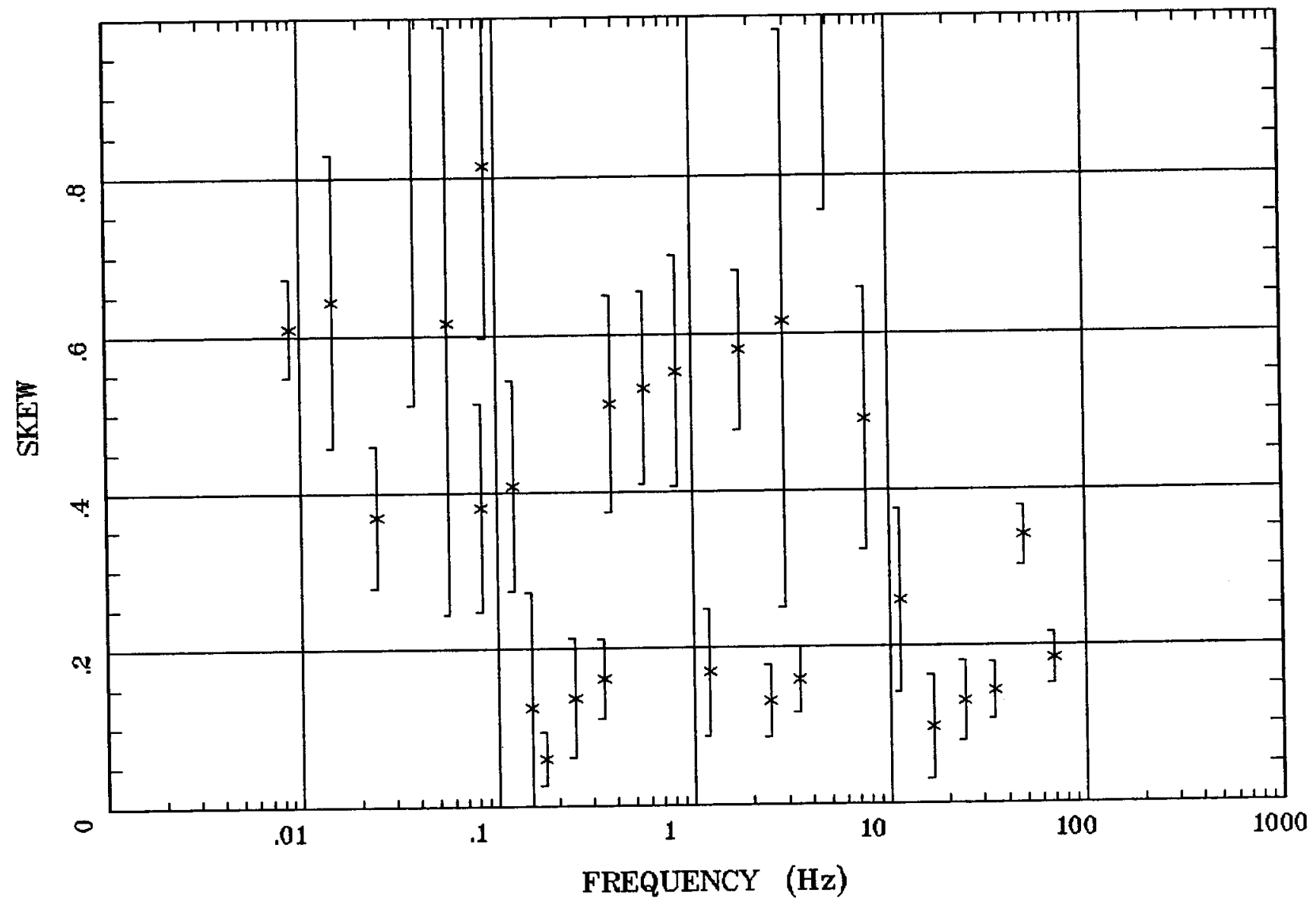

Client: Espanola Basin Remote: none Acquired: 11:3 Jul 17, 2004 Survey Co:USGS
Rotation:

Filename: rr20m25.avg

Channels: Ch1 Ch2 Ch3 Ch4 Ch5 Ch3 Ch4 Plotted: 15:31 Sep 01, 2004

< EMI - ElectroMagnetic Instruments 
E MULT Coh.

Albuquerque NM 1:100K

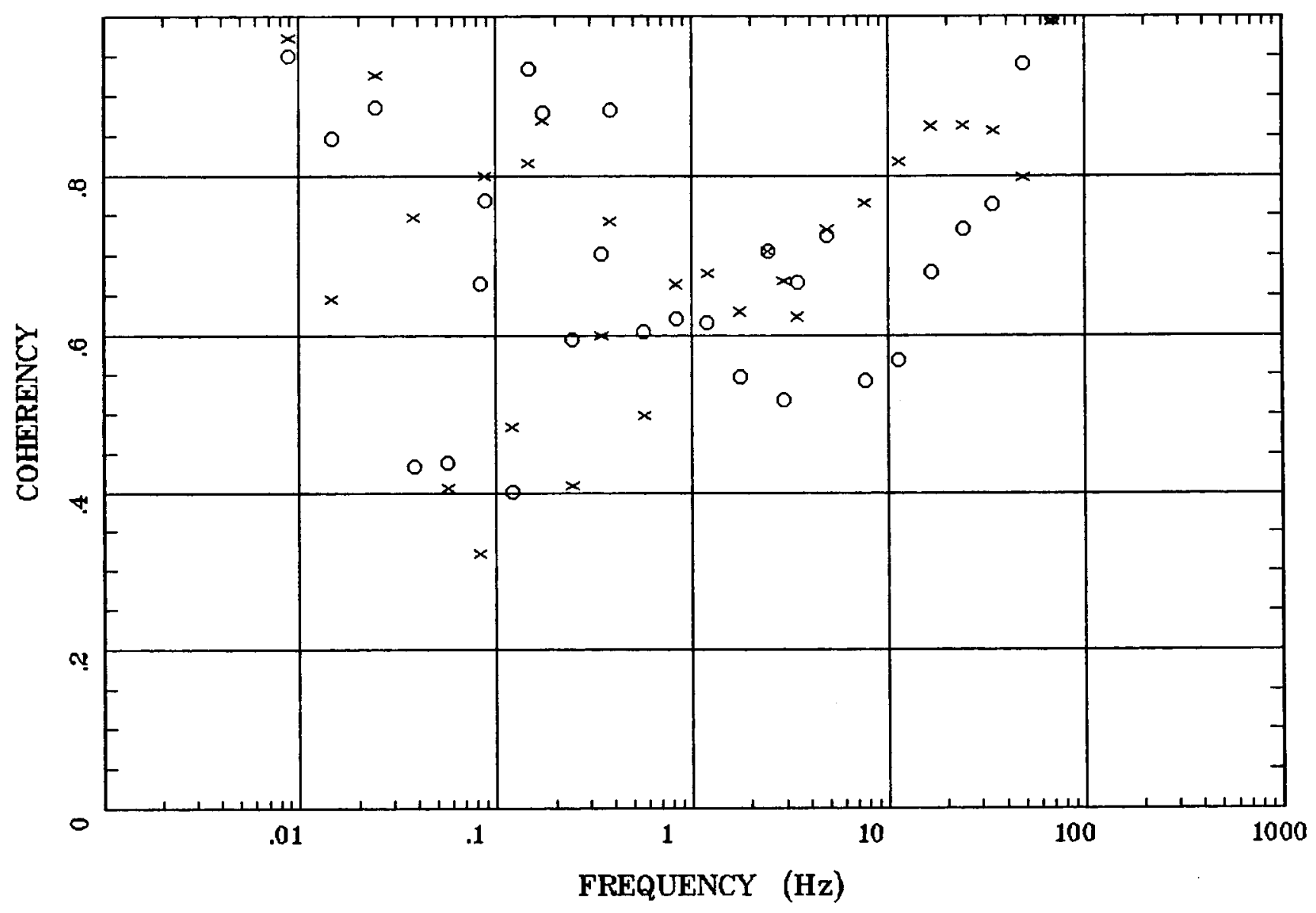

Client: Espanola Basin Remote: none Acquired: 11:3 Jul 17, 2004 Survey Co:USGS
Rotation:

Filename: rr20m25.avg

Channels: Ch1 Ch2 Ch3 Ch4 Ch5 Ch3 Ch4 Plotted: 15:31 Sep 01, 2004

< EMI - ElectroMagnetic Instruments 
Station RR20

Albuquerque NM 1:100K

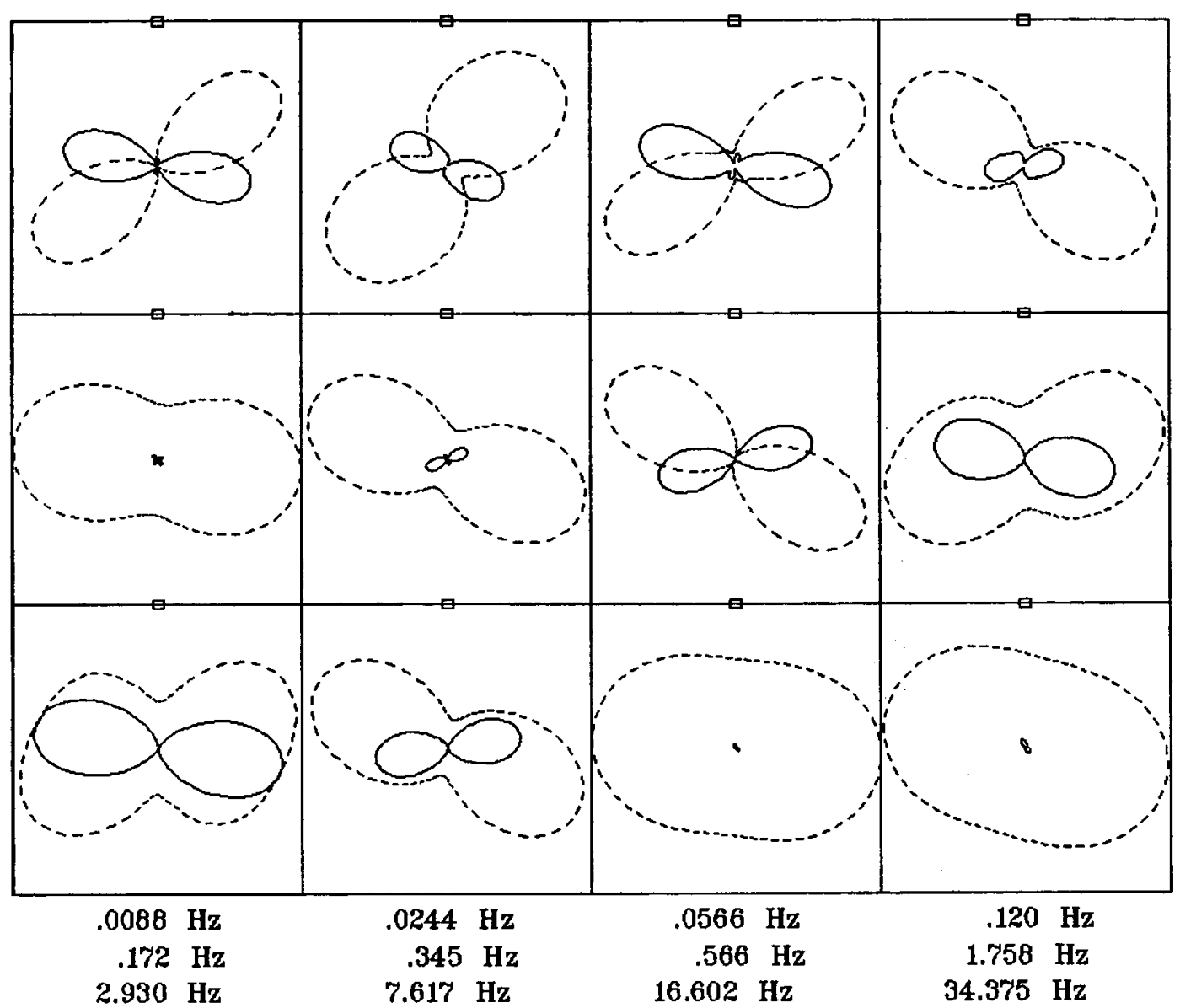

Client: Espanola Basin

Remote: none

Acquired: 11:3 Jul 17, 2004 Survey Co:USGS

\section{Rotation:}

Filename: rr20m25.avg

Channels: Ch1 Ch2 Ch3 Ch4 Ch5 Ch3 Ch4

Plotted: 15:31 Sep 01, 2004

< EMI - ElectroMagnetic Instruments > 


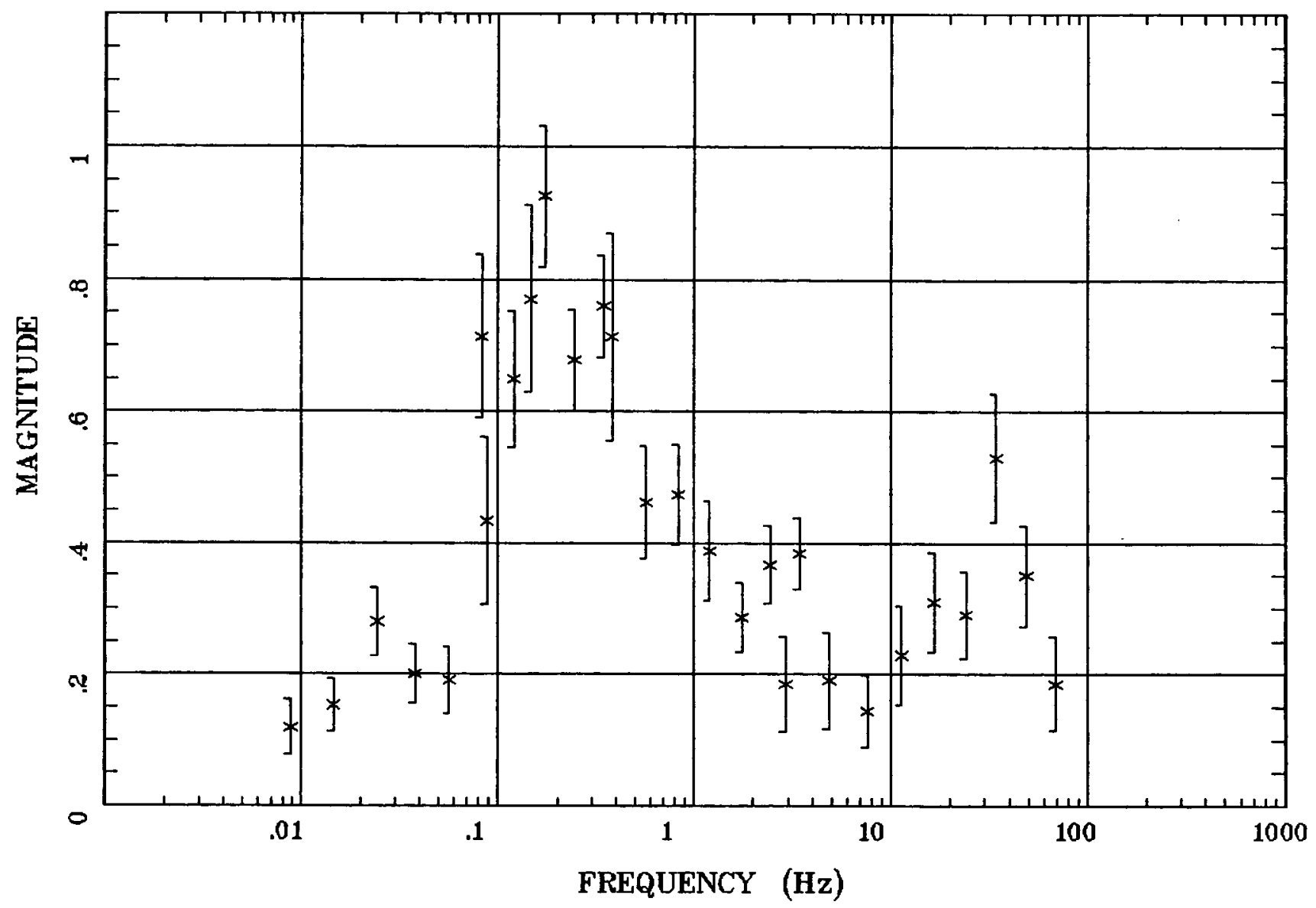

Client: Espanola Basin

Remote: none

Acquired: 11:3 Jul 17, 2004

Survey Co:USGS
Rotation:

Filename: rr20m25.avg

Channels: Ch1 Ch2 Ch3 Ch4 Ch5 Ch3 Ch4

Plotted: 15:31 Sep 01, 2004

< EMI - ElectroMagnetic Instruments 


\section{Station RR20}

TIPPER STRIKE

Albuquerque NM 1:100K

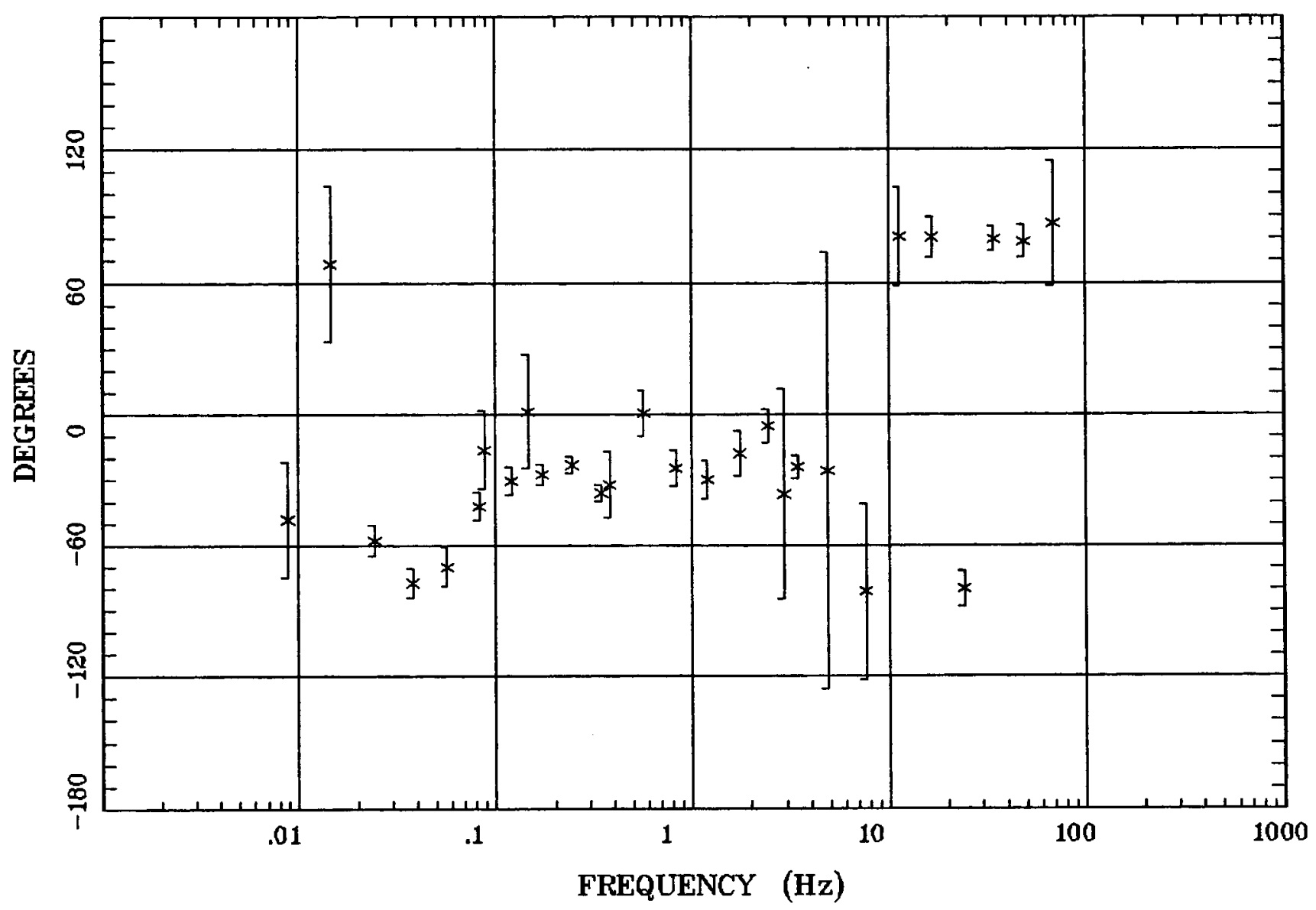

Client: Espanola Basin

Remote: none

Acquired: 11:3 Jul 17, 2004

Survey Co:USGS
Rotation:

Filename: rr20m25.avg

Channels: Ch1 Ch2 Ch3 Ch4 Ch5 Ch3 Ch4

Plotted: 15:31 Sep 01, 2004

< EMI - ElectroMagnetic Instruments 
HzHx.x Coh HzHy.o

Albuquerque NM 1:100K

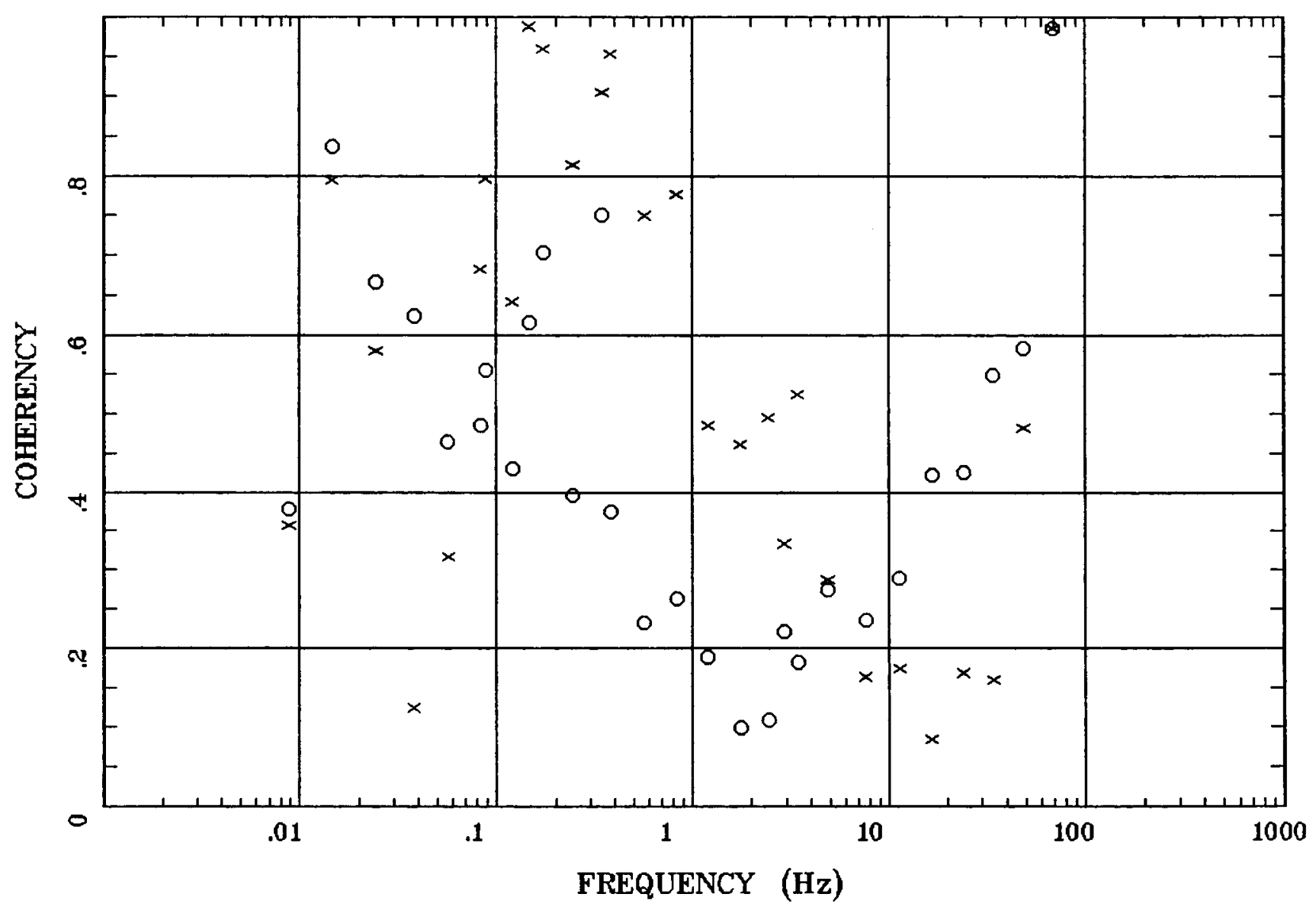

Client: Espanola Basin

Remote: none

Acquired: 11:3 Jul 17, 2004

Survey Co:USGS
Rotation:

Filename: rr20m25.avg

Channels: Ch1 Ch2 Ch3 Ch4 Ch5 Ch3 Ch4

Plotted: 15:31 Sep 01, 2004

< EMI - ElectroMagnetic Instruments 


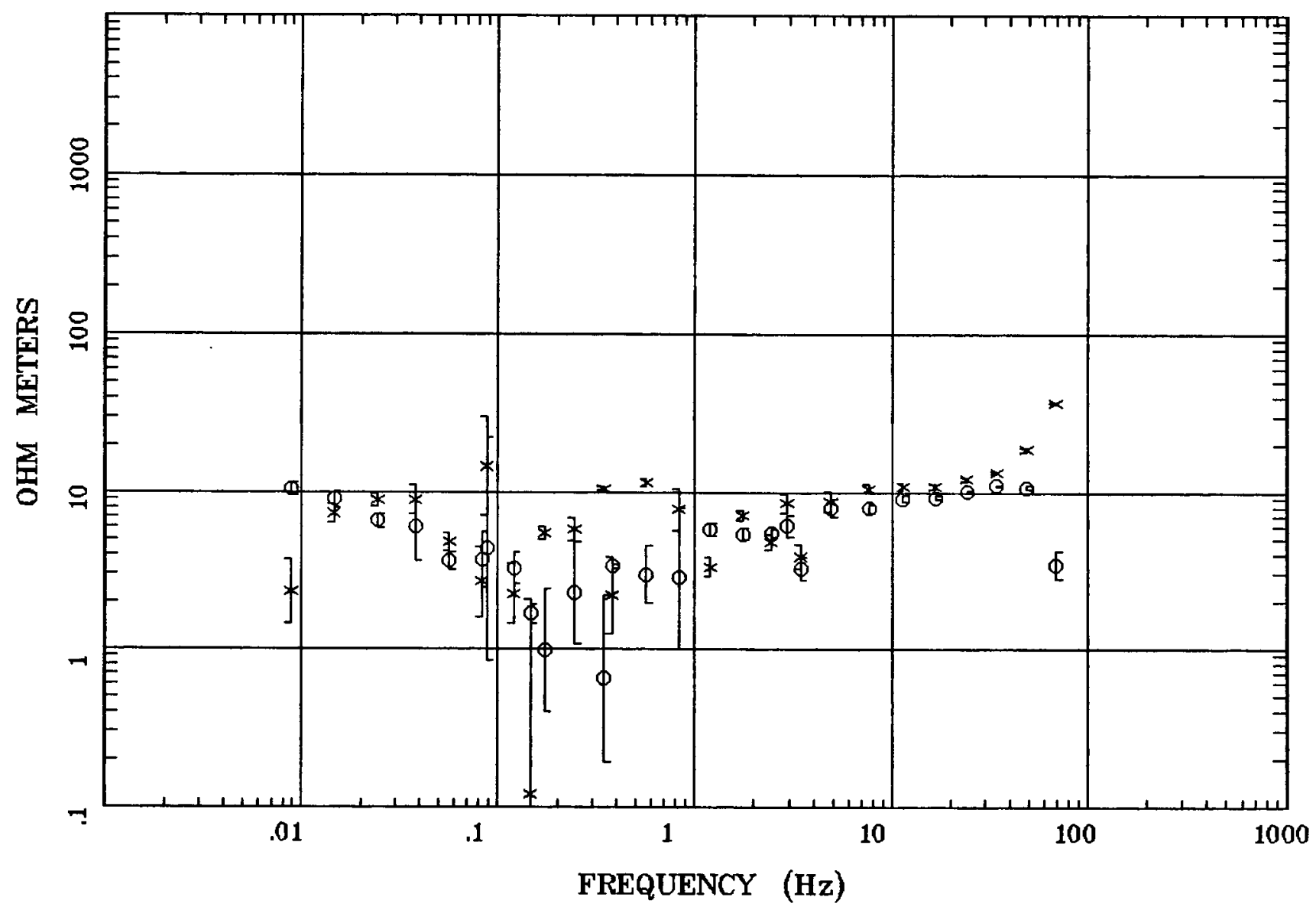

Client: Espanola Basin

Remote: none

Acquired: 10:1 Jul 18, 2004

Survey Co:UsGS
Rotation:

Filename: rr21m13.avg

Channels: Ch1 Ch2 Ch3 Ch4 Ch5 Ch3 Ch4

Plotted: 15:51 Sep 01, 2004

< EMI - ElectroMagnetic Instruments > 


\section{Station RR21}

IMPEDANCE PHASE

Albuquerque NM 1:100K



Client: Espanola Basin

Remote: none

Acquired: 10:1 Jul 18, 2004 Survey Co:USGS
Rotation:

Filename: rr21m13.avg

Channels: Ch1 Ch2 Ch3 Ch4 Ch5 Ch3 Ch4 Plotted: 15:51 Sep 01, 2004

< EMI - ElectroMagnetic Instruments > 


\section{Station RR21}

ROTATION ANGLE

Albuquerque NM $1: 100 \mathrm{~K}$

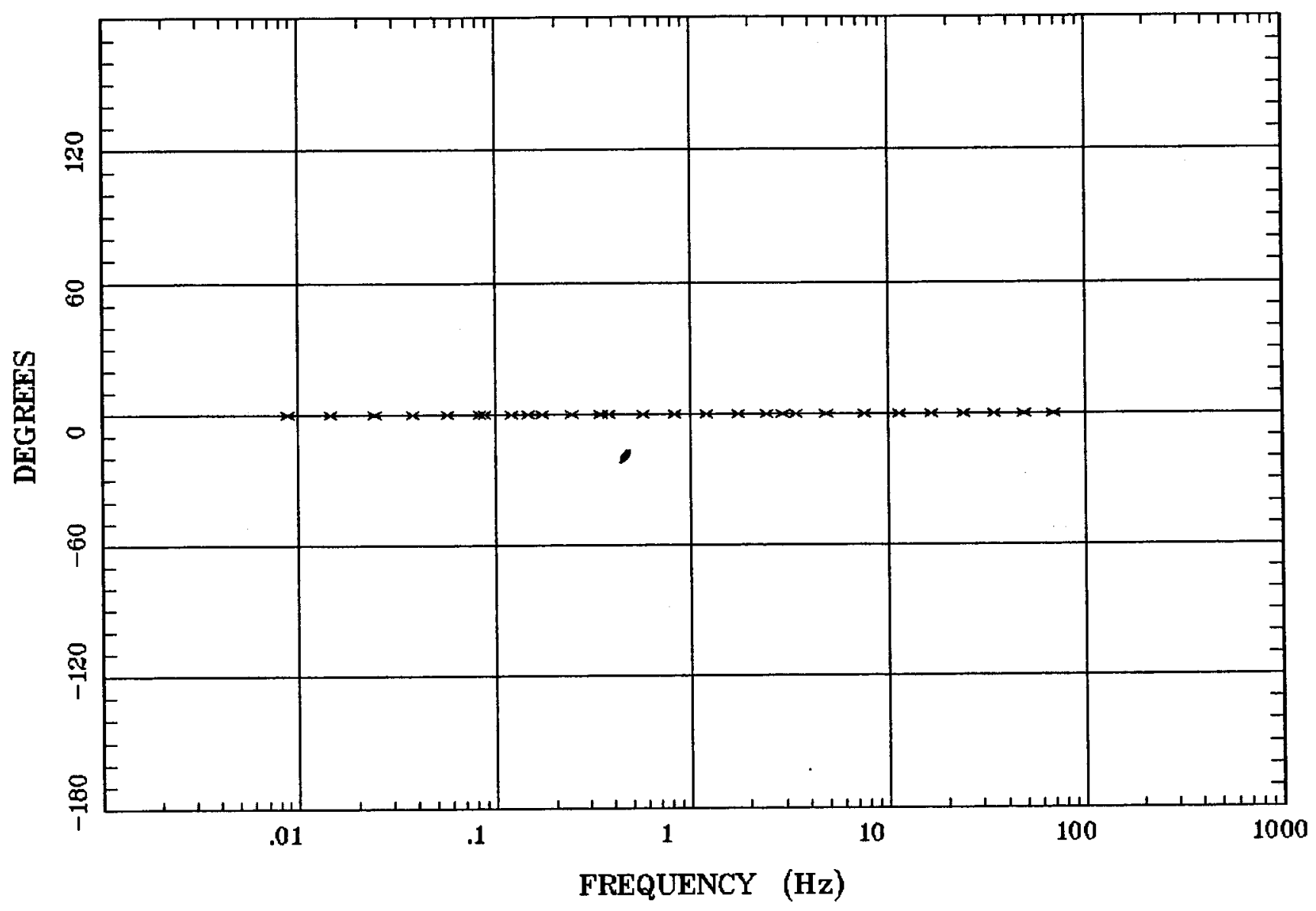

Client: Espanola Basin

Remote: none

Acquired: 10:1 Jul 18, 2004 Survey Co:USGS
Rotation:

Filename: $\operatorname{rr} 1 \mathrm{~m} 13 . \mathrm{avg}$

Channels: Ch1 Ch2 Ch3 Ch4 Ch5 Ch3 Ch4 Plotted: 15:51 Sep 01, 2004

$<$ EMI - ElectroMagnetic Instruments 


\section{Station RR21}

IMPEDANCE SKEW

Albuquerque NM 1:100K



Client: Espanola Basin

Rotation:

Remote: none

Acquired: 10:1 Jul 18, 2004

Survey Co:USGS

Filename: rr21m13.avg

Channels: Ch1 Ch2 Ch3 Ch4 Ch5 Ch3 Ch4

Plotted: 15:51 Sep 01, 2004

$<$ EMI - ElectroMagnetic Instruments 
E MULT Coh.

Albuquerque NM 1:100K



Client: Espanola Basin Remote: none

Acquired: 10:1 Jul 18, 2004 Survey Co:USGS
Rotation:

Filename: rr21m13.avg

Channels: Ch1 Ch2 Ch3 Ch4 Ch5 Ch3 Ch4

Plotted: 15:52 Sep 01, 2004

< EMI - ElectroMagnetic Instruments > 
Station RR21

POLAR PLOTS Albuquerque NM 1:100K



Client: Espanola Basin

Remote: none

Acquired: 10:1 Jul 18, 2004 Survey Co:USGS
Rotation:

Filename: rr21m13.avg

Channels: Ch1 Ch2 Ch3 Ch4 Ch5 Ch3 Ch4

Plotted: 15:52 Sep 01, 2004

< EMI - ElectroMagnetic Instruments > 


\section{Station RR21}

TIPPER MAGNITUDE

Albuquerque NM 1:100K



Client: Espanola Basin

Remote: none

Acquired: 10:1 Jul 18, 2004

Survey Co:USGS
Rotation:

Filename: rr21m13.avg

Channels: Ch1 Ch2 Ch3 Ch4 Ch5 Ch3 Ch4

Plotted: 15:52 Sep 01, 2004

$<$ EMI - ElectroMagnetic Instruments 


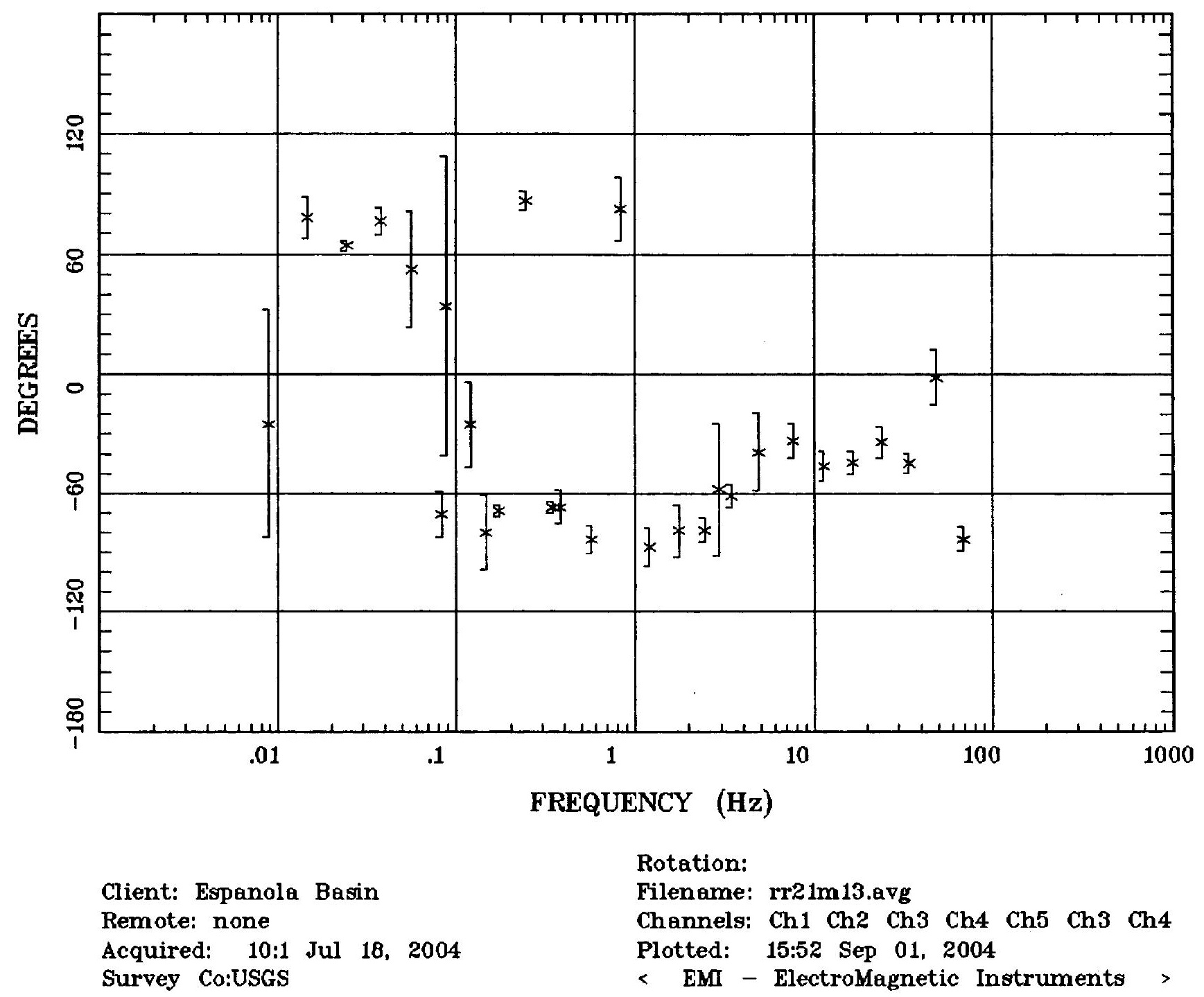


Station RR21



Client: Espanola Basin Remote: none Acquired: 10:1 Jul 18, 2004 Survey Co:USGS
Rotation:

Filename: rr21m13.avg

Channels: Ch1 Ch2 Ch3 Ch4 Ch5 Ch3 Ch4 Plotted: 15:52 Sep 01, 2004

< EMI - ElectroMagnetic Instruments 




Client: Espanala Basin Remote: none Acquired: 10:4 Jul 21, 2004 Survey Co:USGS

\section{Rotation:}

Filename: rrazm.avg

Channels: Ch1 Ch2 Ch3 Ch4 Ch5 Ch3 Ch4 Plotted: 08:08 Aug 09, 2004

< EMI - ElectroMagnetic Instruments 


\section{Station RR22}

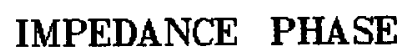

Albuquerque NM 1:100K

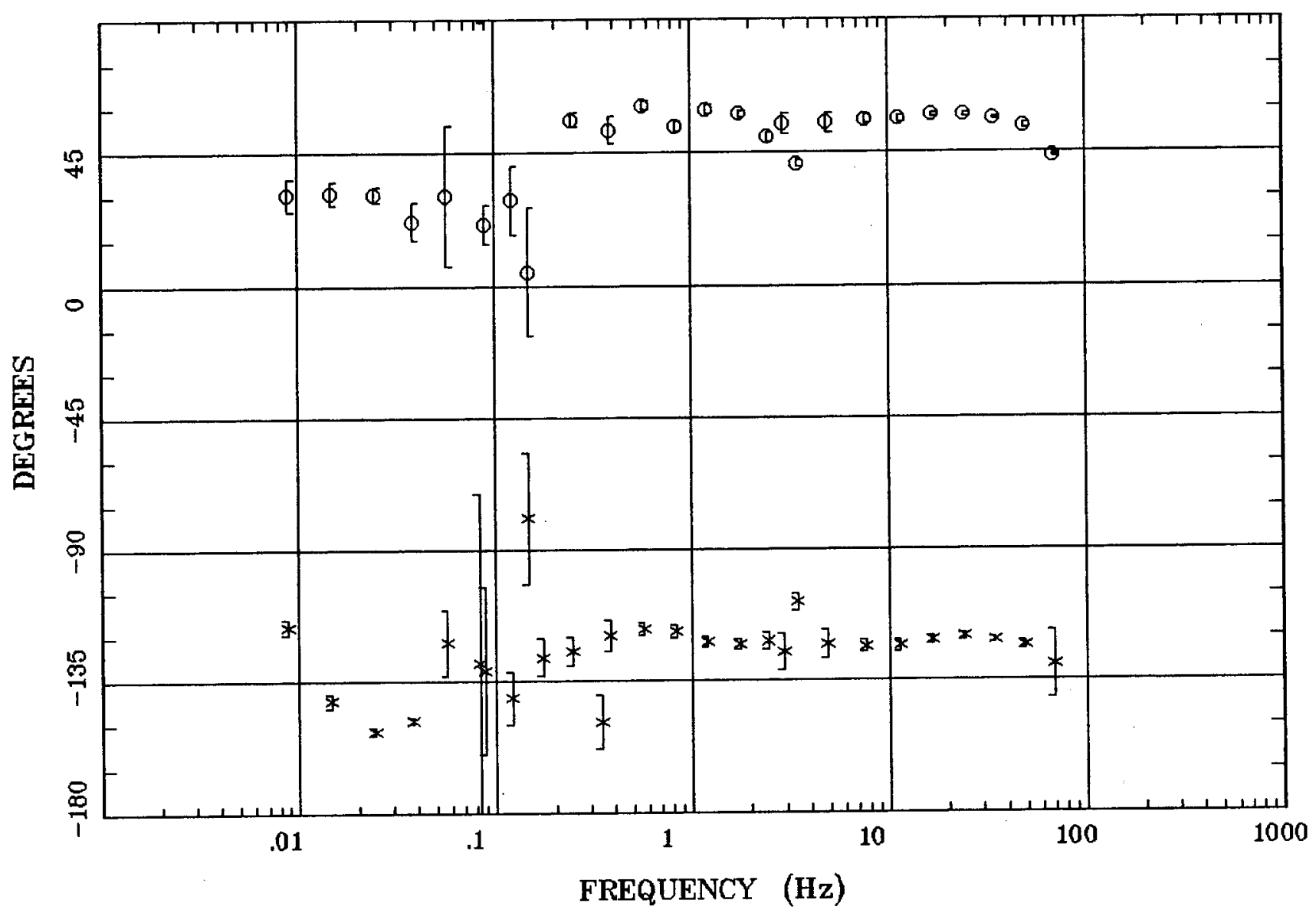

Client: Espanola Basin Remote: none Acquired: 10:4 Jul 21, 2004 Survey Co:USGS
Rotation:

Filename: rr22m.avg

Channels: Ch1 Ch2 Ch3 Ch4 Ch5 Ch3 Ch4 Plotted: 08:08 Aug 09, 2004

< EMI - ElectroMagnetic Instruments 


\section{Station RR22}

\section{ROTATION ANGLE}

Albuquerque NM 1:100K

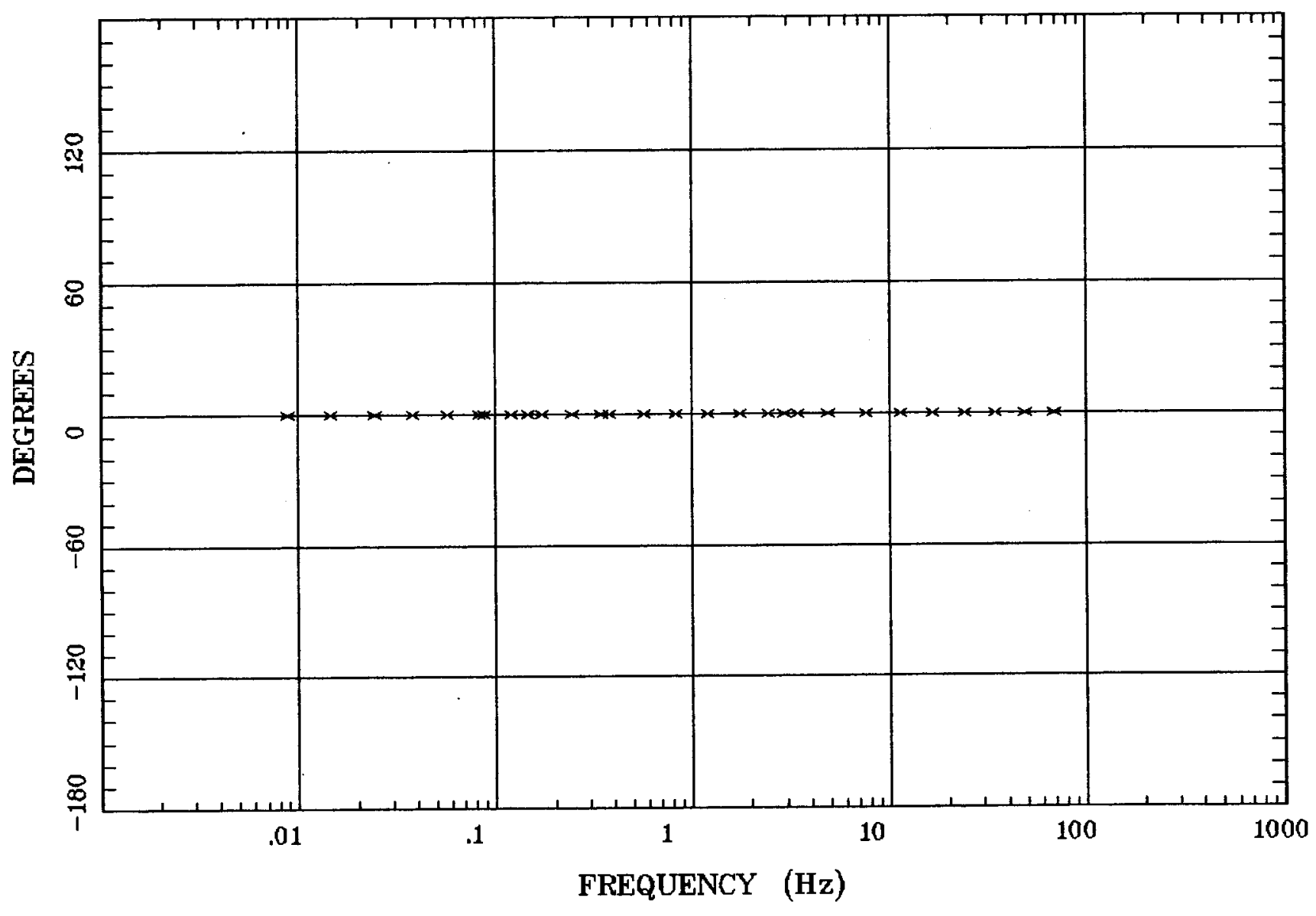

Client: Espanola Basin

Remote: none

Acquired: 10:4 Jul 21, 2004

Survey Co:USGS
Rotation:

Filename: rrazm.avg

Channels: Ch1 Ch2 Ch3 Ch4 Ch5 Ch3 Ch4

Platted: 08:08 Aug 09, 2004

< EMI - ElectroMagnetic Instruments > 


\section{Station RR22}

\section{IMPEDANCE SKEW}

Albuquerque NM 1:100K

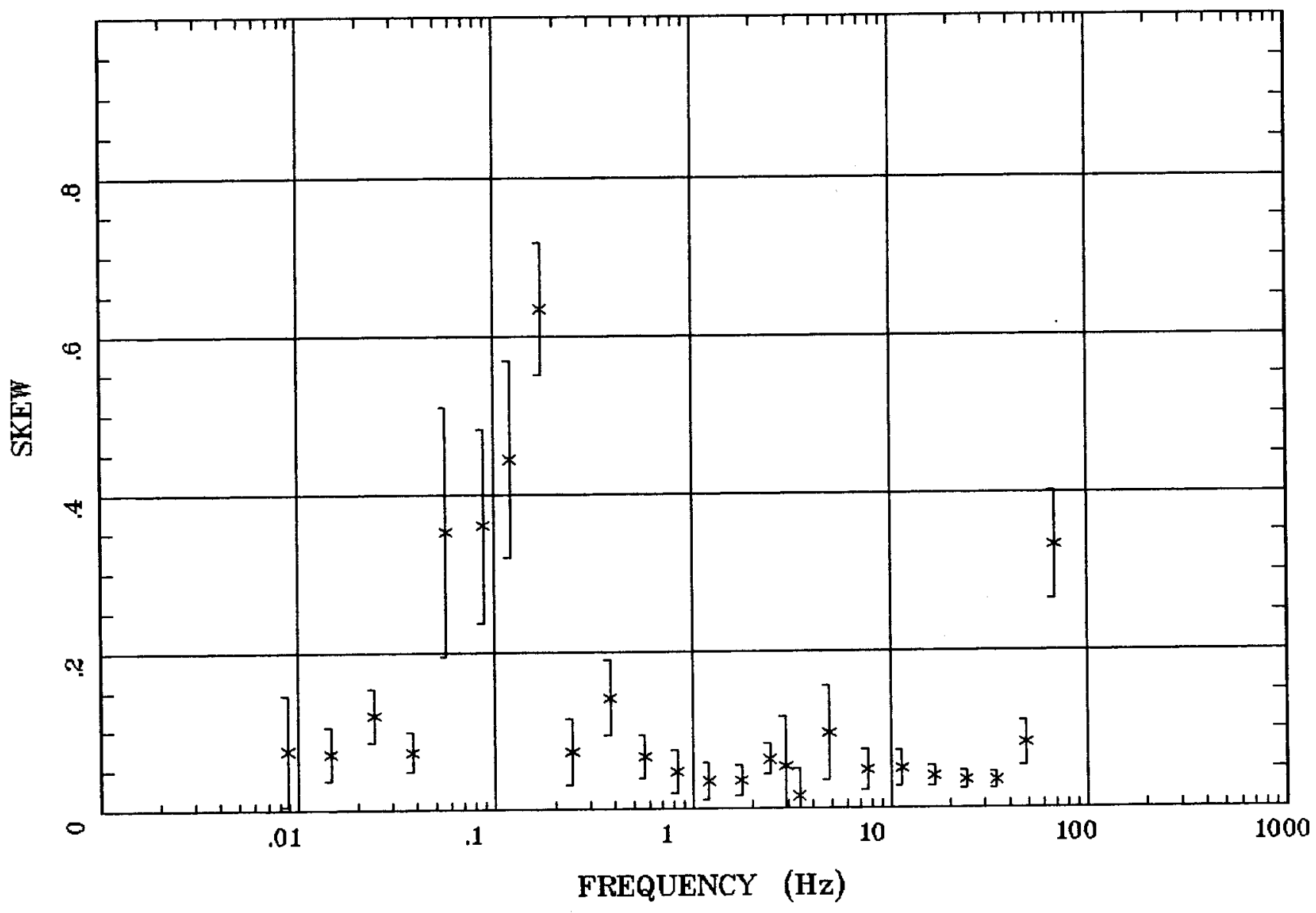

Client: Espanola Besin

Remote: none

Acquired: 10:4 Jul 21, 2004 Survey Co:USGS
Rotation:

Filename: rr22m.avg

Channels: Ch1 Ch2 Ch3 Ch4 Ch5 Ch3 Ch4

Plotted: 08:08 Aug 09, 2004

<EMI - ElectroMagnetic Instruments > 


\section{E MULT Coh.}

Albuquerque NM 1:100K

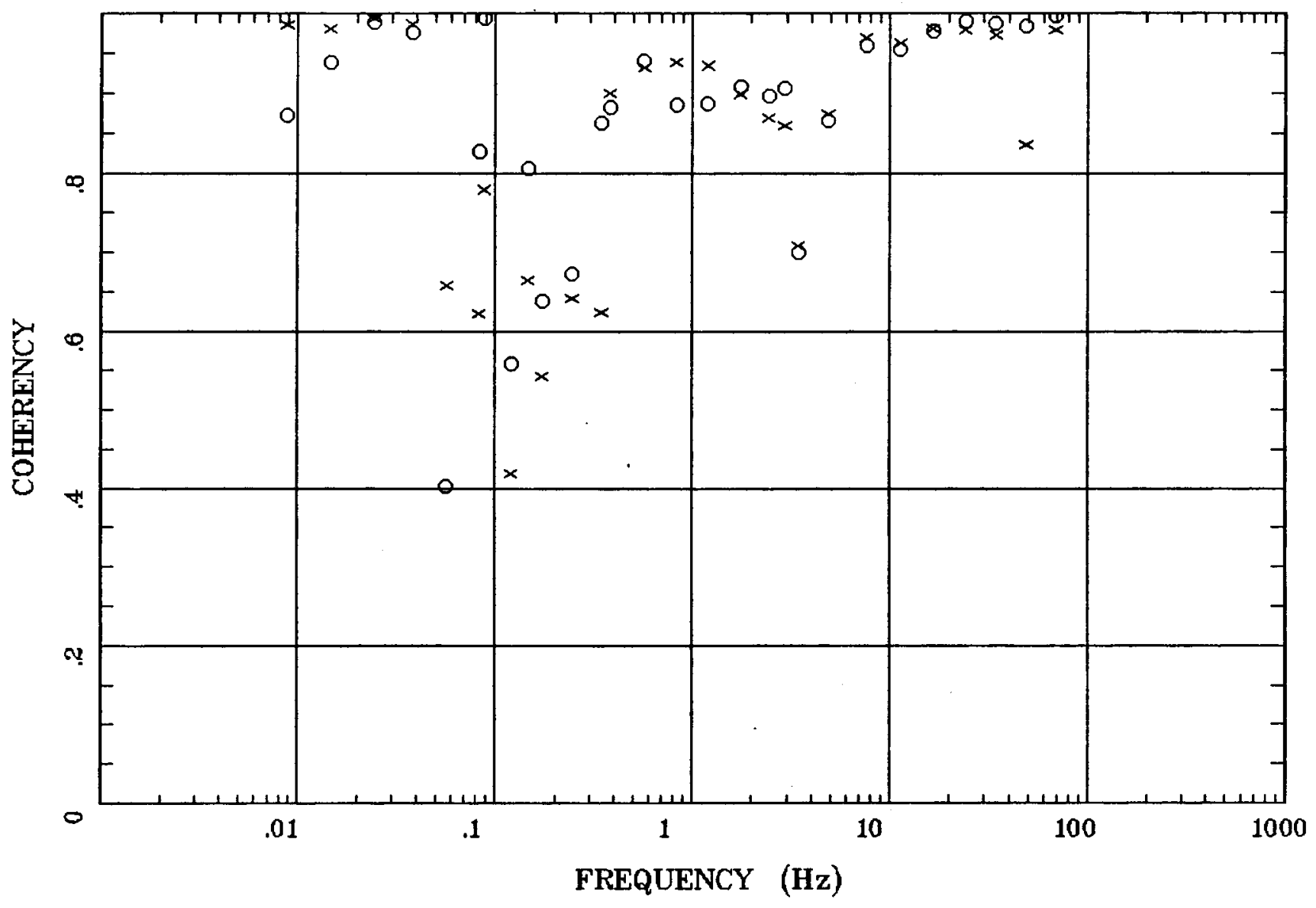

Client: Espanola Basin

Remote: none

Acquired: 10:4 Jul 21, 2004

Survey Co:USGS
Rotation:

Filename: rr22m.avg

Channels: Ch1 Ch2 Ch3 Ch4 Ch5 Ch3 Ch4 Plotted: 08:08 Aug 09, 2004

$<$ EMI - ElectroMagnetic Instruments 


\section{Station RR22}

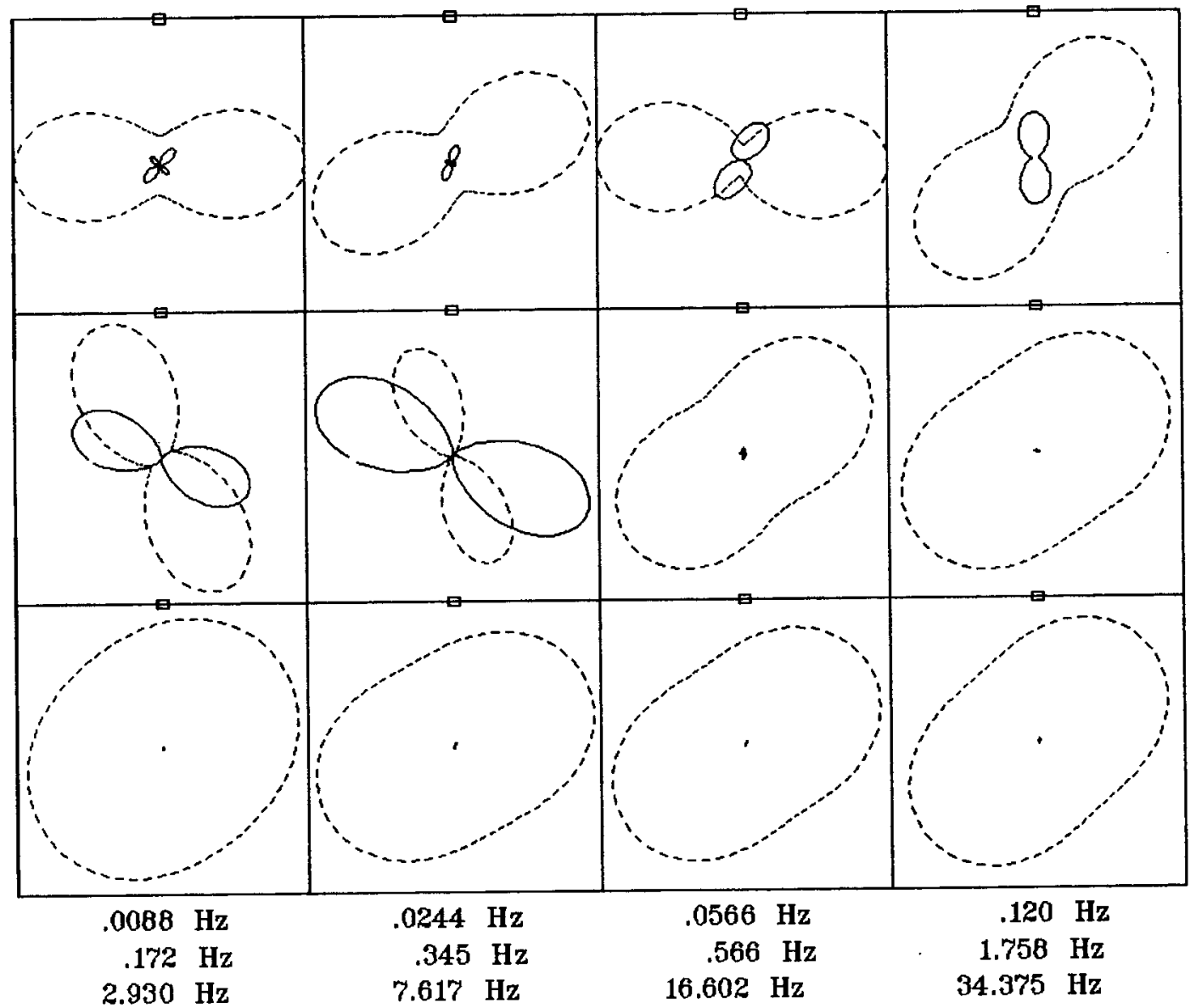

Client: Espanola Basin Remote: none

Acquired: 10:4 Jul 21, 2004 Survey Co:USGS
Rotation:

Filename: rrz2m.avg

Channels: Ch1 Ch2 Ch3 ch4 Ch5 Ch3 Ch4 Plotted: 08:09 Aug 09, 2004

< EMI - ElectroMagnetic Instruments > 


\section{Station RR22}

TIPPER MAGNITUDE

Albuquerque NM 1:100K

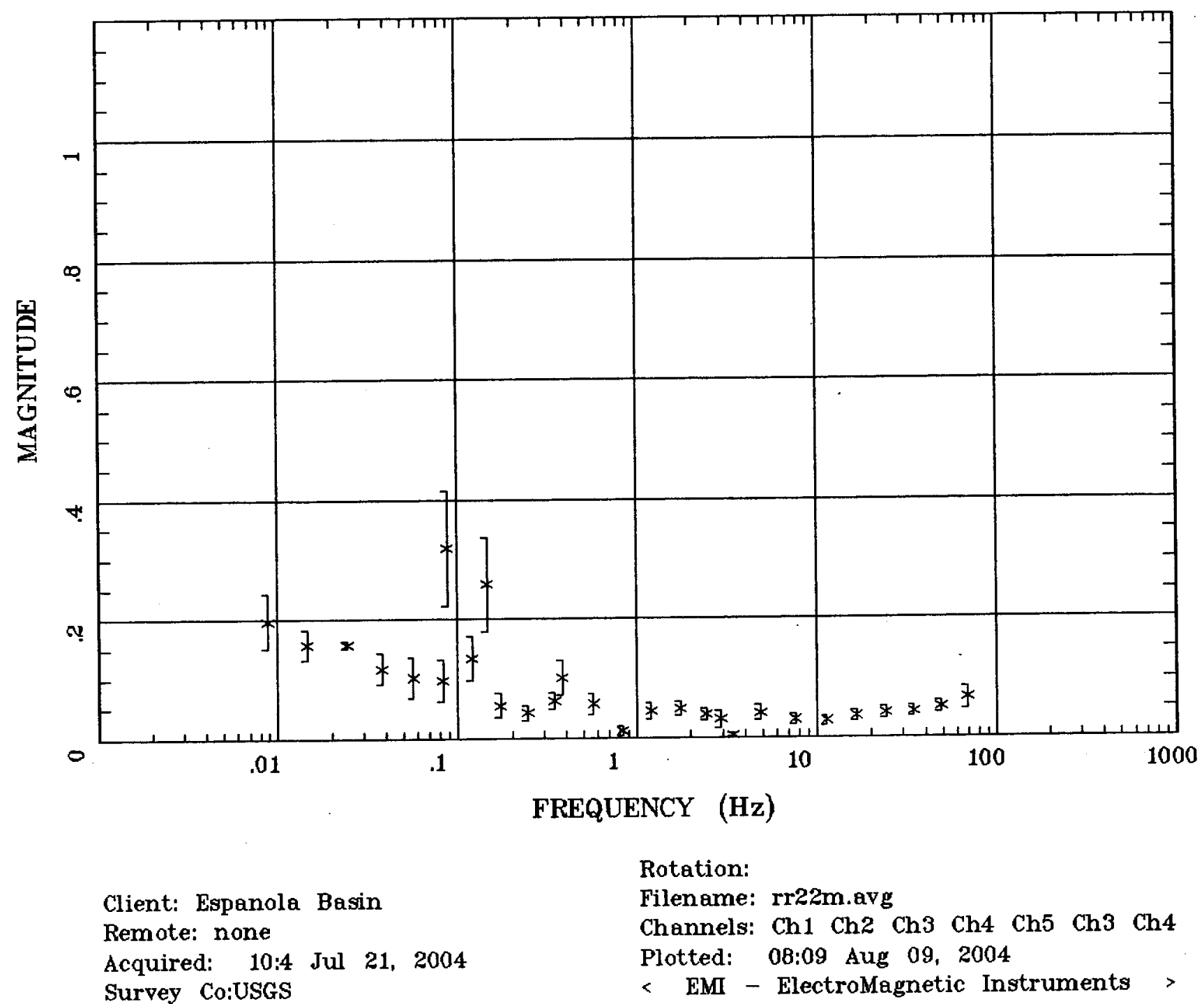




\section{Station RR22}

TIPPER STRIKE

Albuquerque NM 1:100K

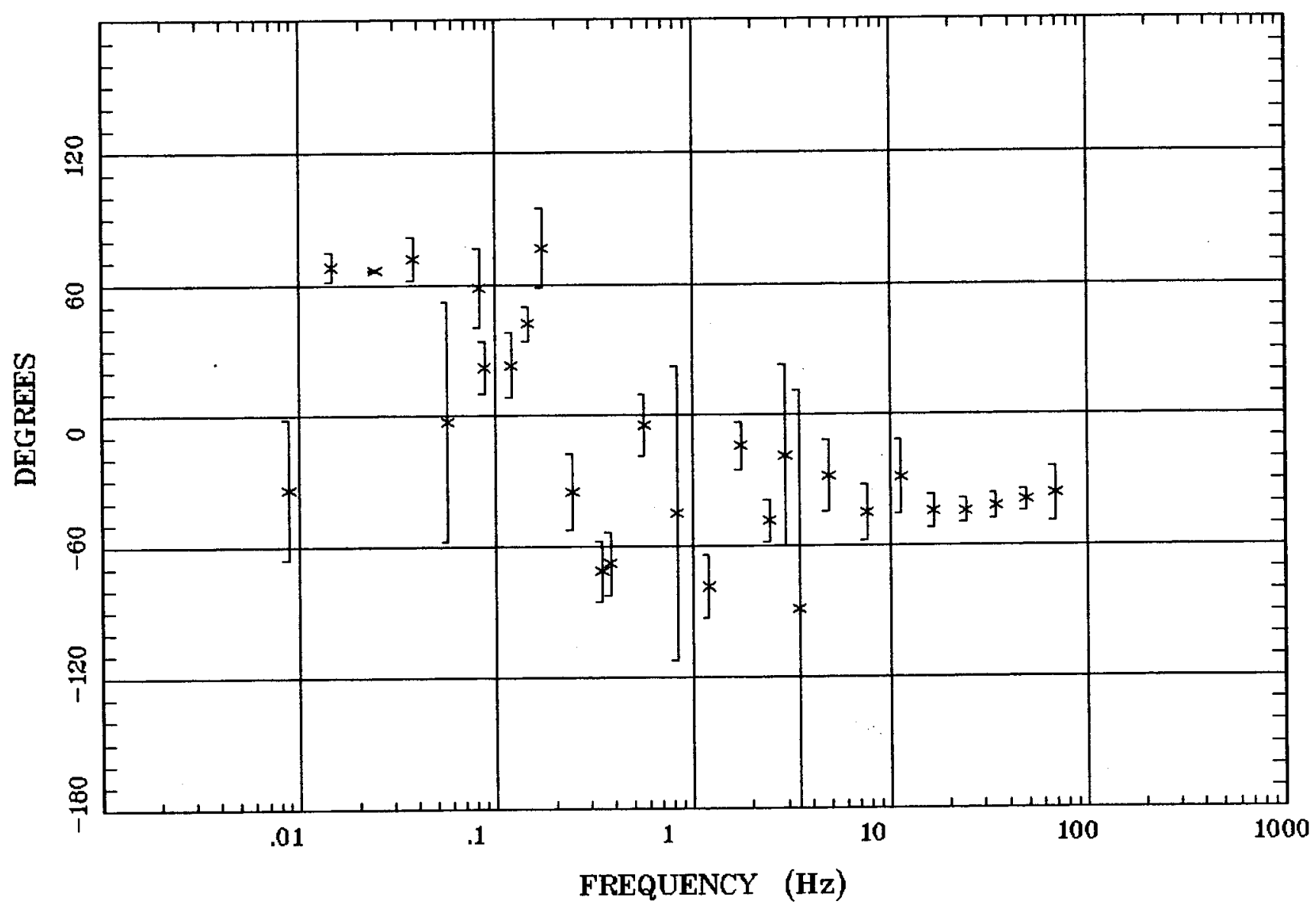

Client: Espanola Basin Remote: none Acquired: 10:4 Jul 21, 2004 Survey Co:USGS
Rotation:

Filename: rr22m.avg

Channels: Ch1 Ch2 Ch3 Ch4 Ch5 Ch3 Ch4 Plotted: 08:09 Aug 09, 2004

< EMI - ElectroMagnetic Instruments > 
Albuquerque NM 1:100K

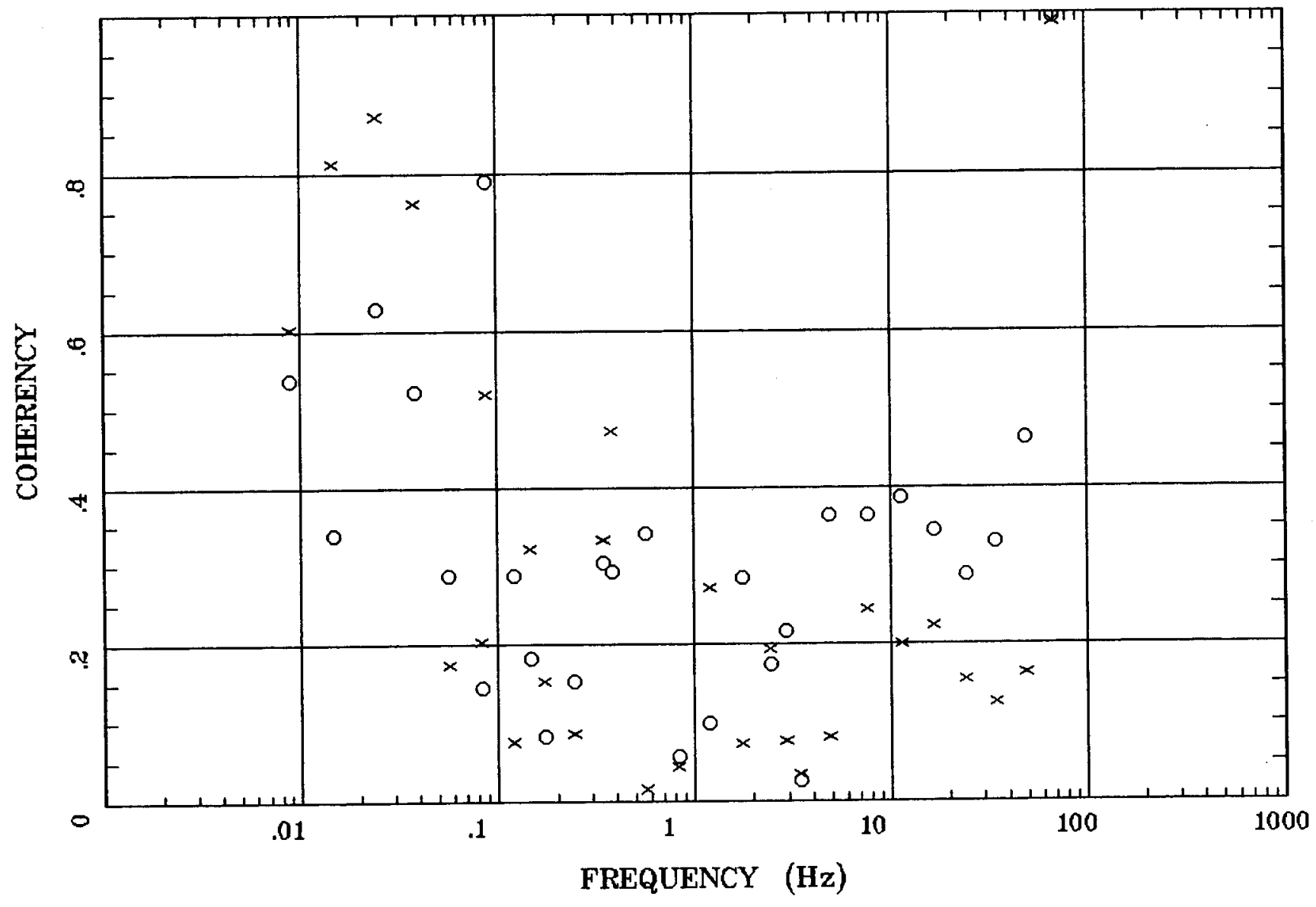

Client: Espanola Basin

Remote: none

Acquired: 10:4 Jul 21, 2004

Survey Co:USGS
Rotation:

Filename: rrz2m.avg

Channels: Ch1 Ch2 Ch3 ch4 Ch5 Ch3 Ch4

Plotted: 08:09 Aug 09, 2004

< EMI - ElectroMagnetic Instruments > 


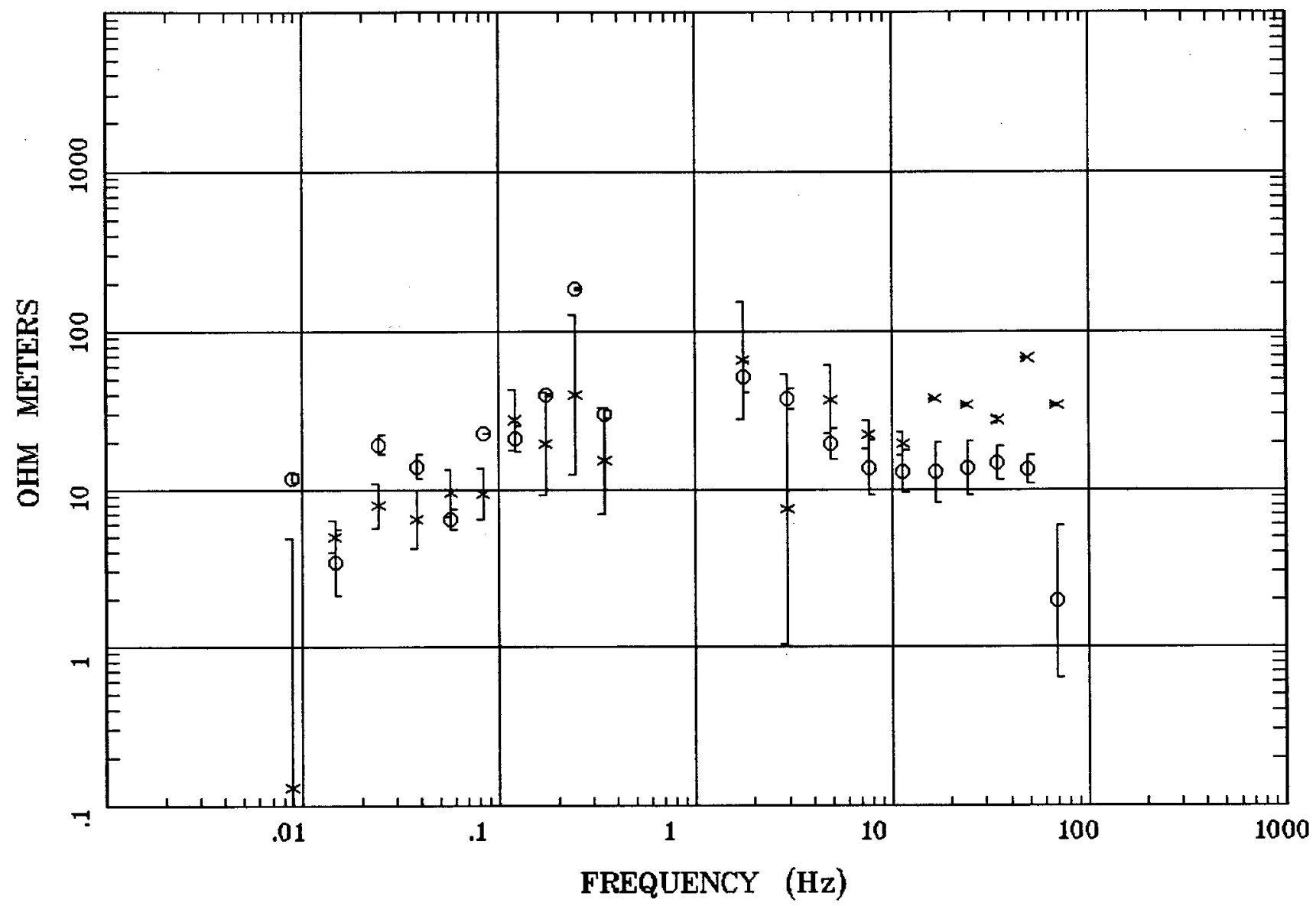

Client: Espanola Basin

Remote: none

Acquired: 11:2 Jul 19, 2004

Survey Co:USGS
Rotation:

Filename: rr23m.avg

Channels: Ch1 Ch2 Ch3 Ch4 Ch5 Ch3 Ch4 Plotted: 11:44 Jan 12, 2006

$<$ EMI - ElectroMagnetic Instruments 




Client: Espanola Basin

Remote: none

Acquired: 11:2 Jul 19, 2004

Survey Co:USGS
Rotation:

Filename: rr23m.avg

Channels: Ch1 Ch2 Ch3 Ch4 Ch5 Ch3 Ch4 Plotted: 11:44 Jan 12, 2006

< EMI - ElectroMagnetic Instruments > 


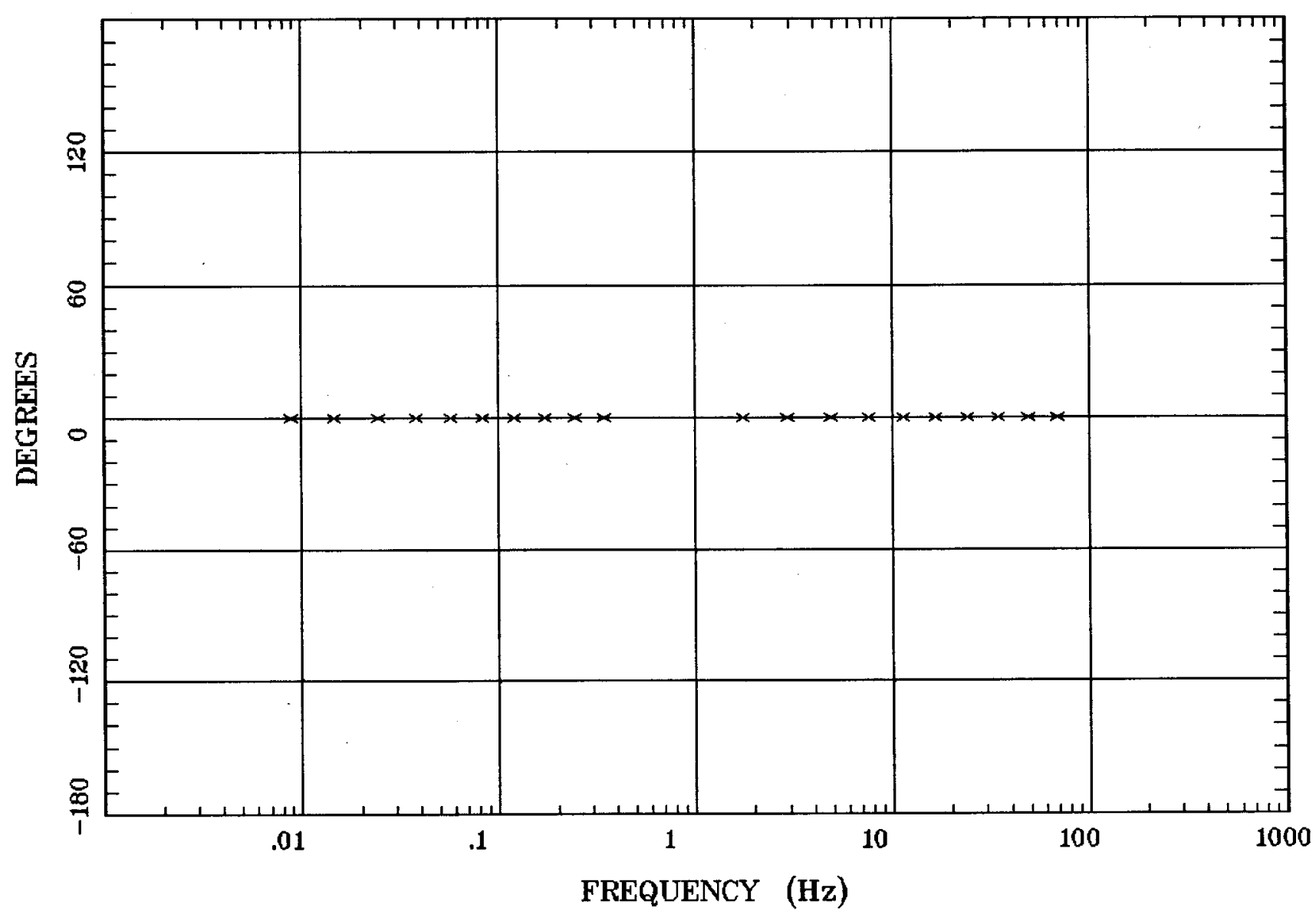

Client: Espanola Basin

Remote: none

Acquired: 11:2 Jul 19, 2004

Survey Co:USGS
Rotation:

Filename: rr23m.avg

Channels: Ch1 Ch2 Ch3 Ch4 Ch5 Ch3 Ch4

Plotted: 11:44 Jan 12, 2006

< EMI - ElectroMagnetic Instruments 


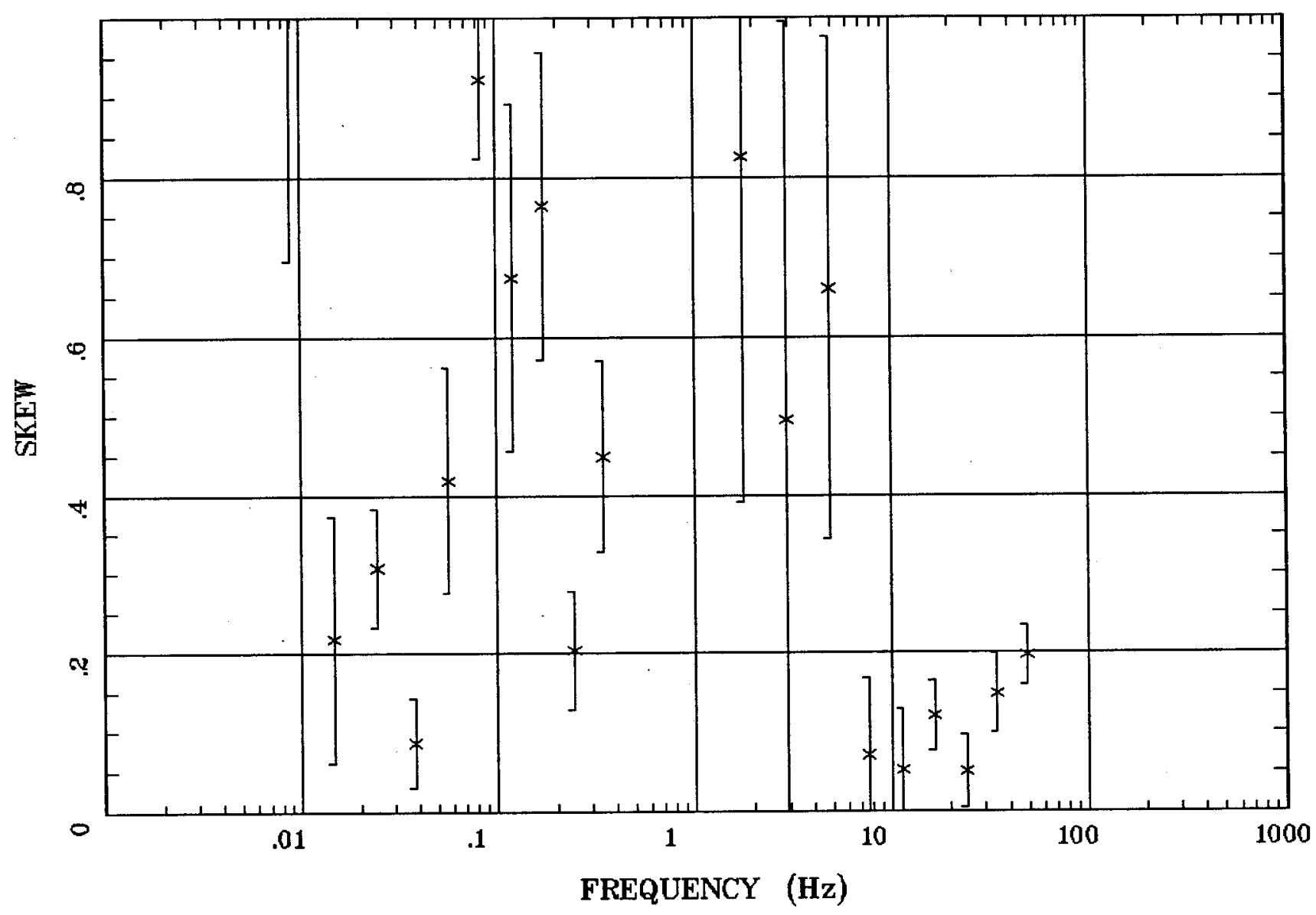

Client: Espanola Basin Remote: none

Acquired: 11:2 Jul 19, 2004

Survey Co:USGS
Rotation:

Filename: rr23m.avg

Channels: Ch1 Ch2 Ch3 Ch4 Ch5 Ch3 Ch4 Plotted: 11:44 Jan 12, 2006

< EMI - ElectroMagnetic Instruments > 


\section{E MULT Coh.}

Station RR23

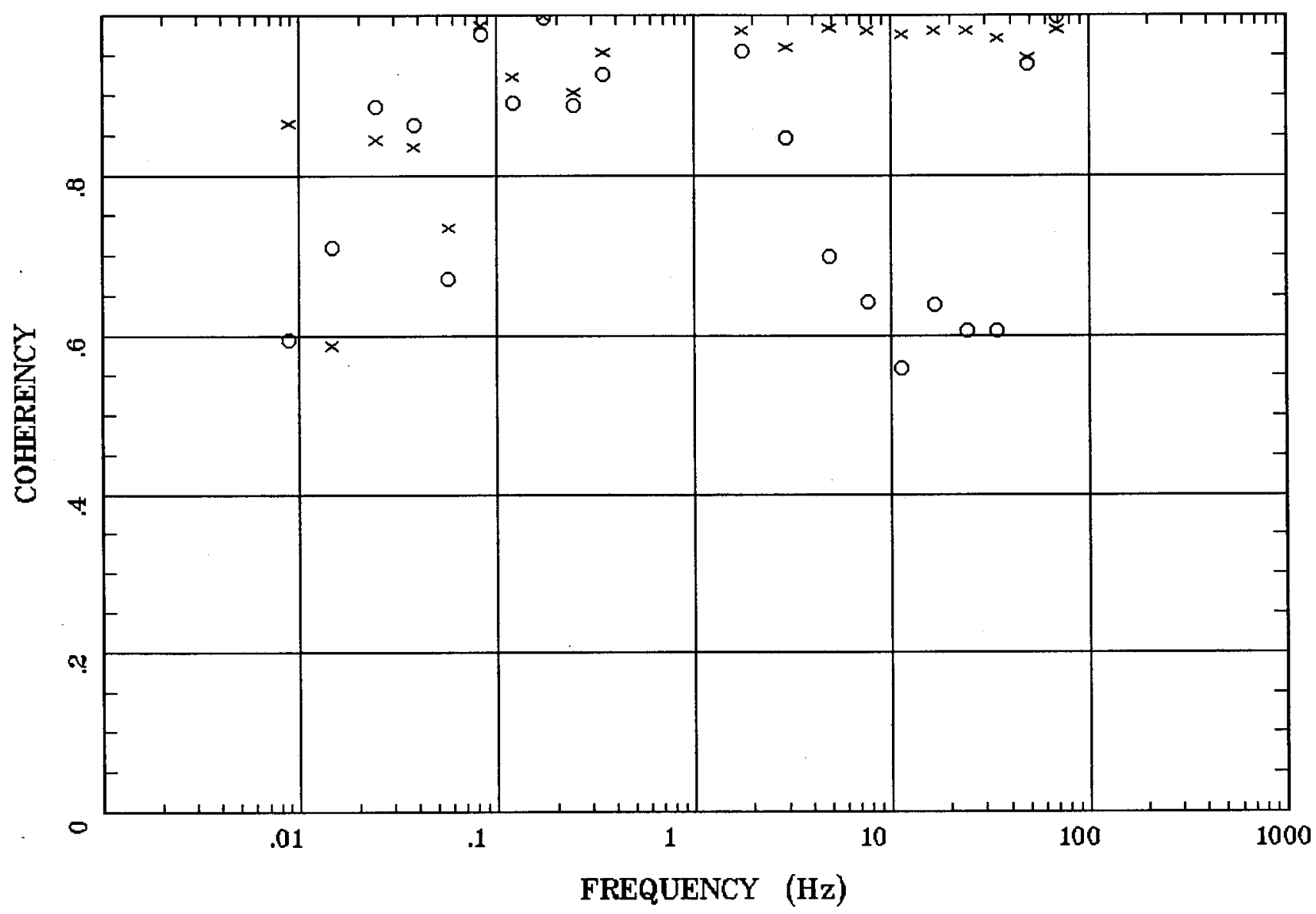

Client: Espanala Basin

Remote: none

Acquired: 11:2 Jul 19, 2004

Survey Co:USGS
Rotation:

Filename: rr29m.avg

Channels: Ch1 Ch2 Ch3 Ch4 Ch5 Ch3 Ch4

Plotted: 11:44 Jan 12, 2006

< EMI - ElectroMagnetic Instruments > 


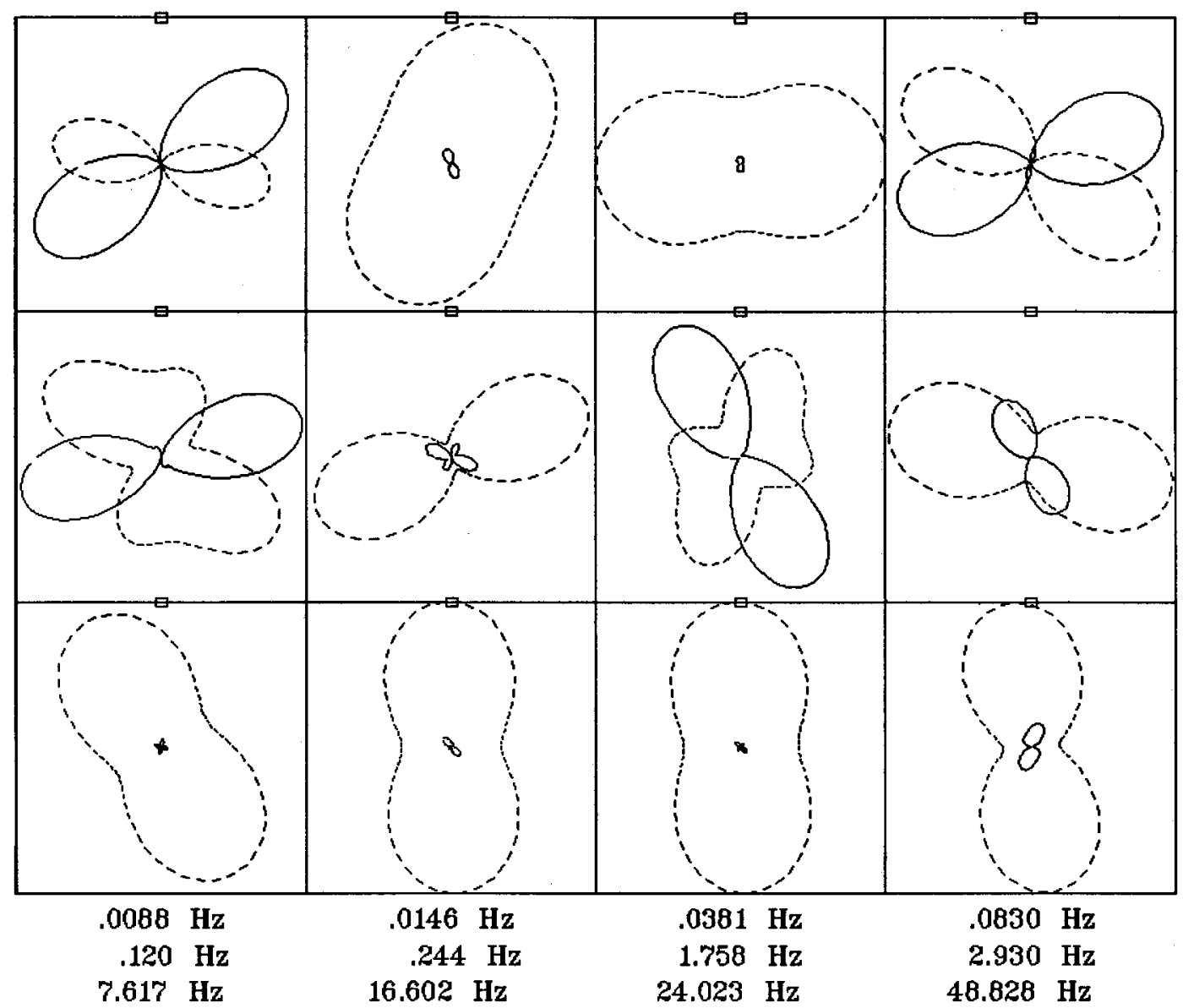

Client: Espanola Basin

Remote: none

Acquired: 11:2 Jul 19, 2004

Survey Co:USGS
Rotation:

Filename: rr23m.avg

Channels: Ch1 Ch2 Ch3 Ch4 Ch5 Ch3 Ch4

Plotted: 11:44 Jan 12, 2006

<EMI - ElectroMagnetic Instruments > 


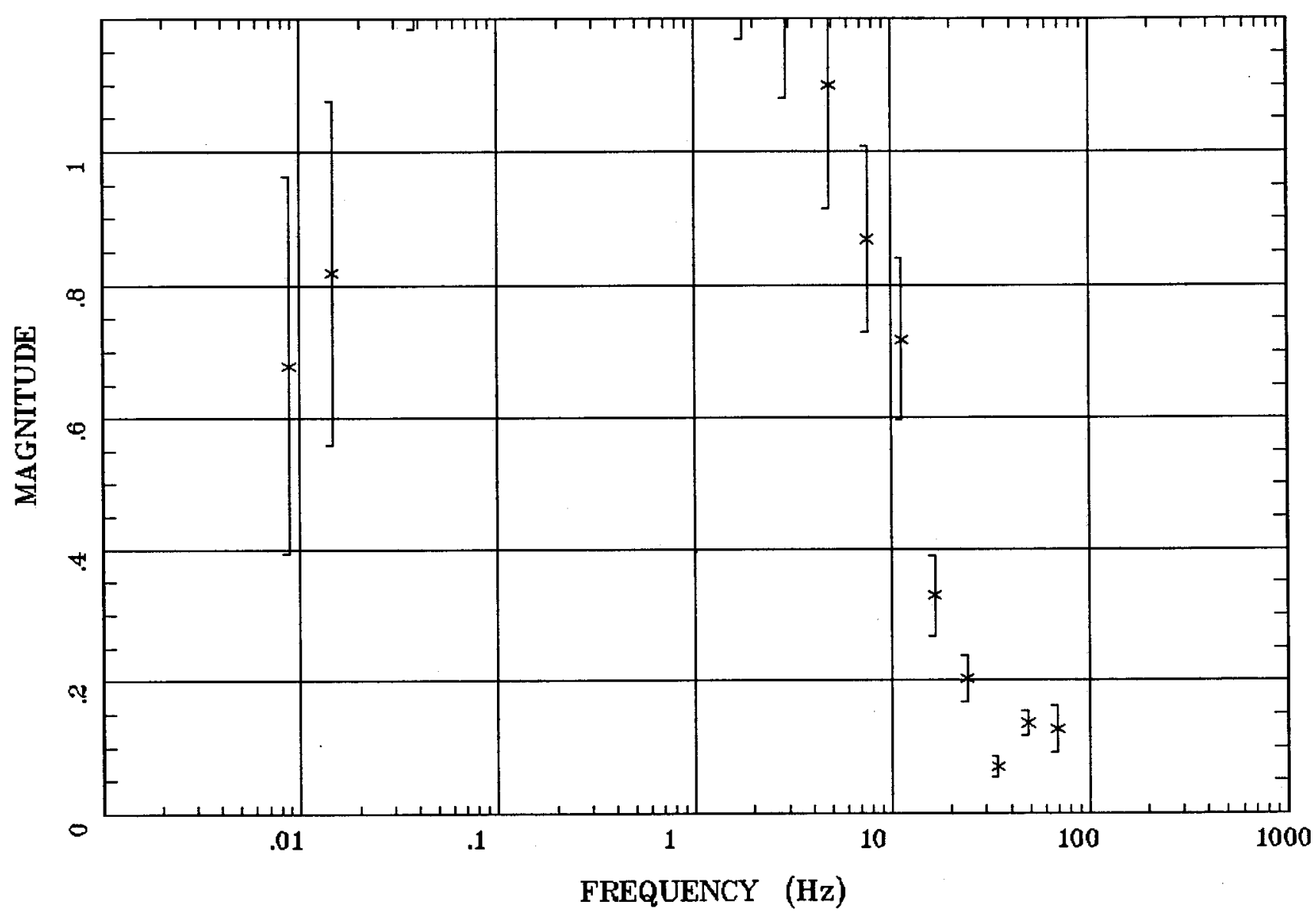

Client: Espanola Basin

Remote: none

Acquired: 11:2 Jul 19, 2004

Survey Co:USGS
Rotation:

Filename: rr23m.avg

Channels: Ch1 Ch2 Ch3 Ch4 Ch5 Ch3 Ch4

Plotted: 11:44 Jan 12, 2006

< EMI - ElectroMagnetic Instruments > 


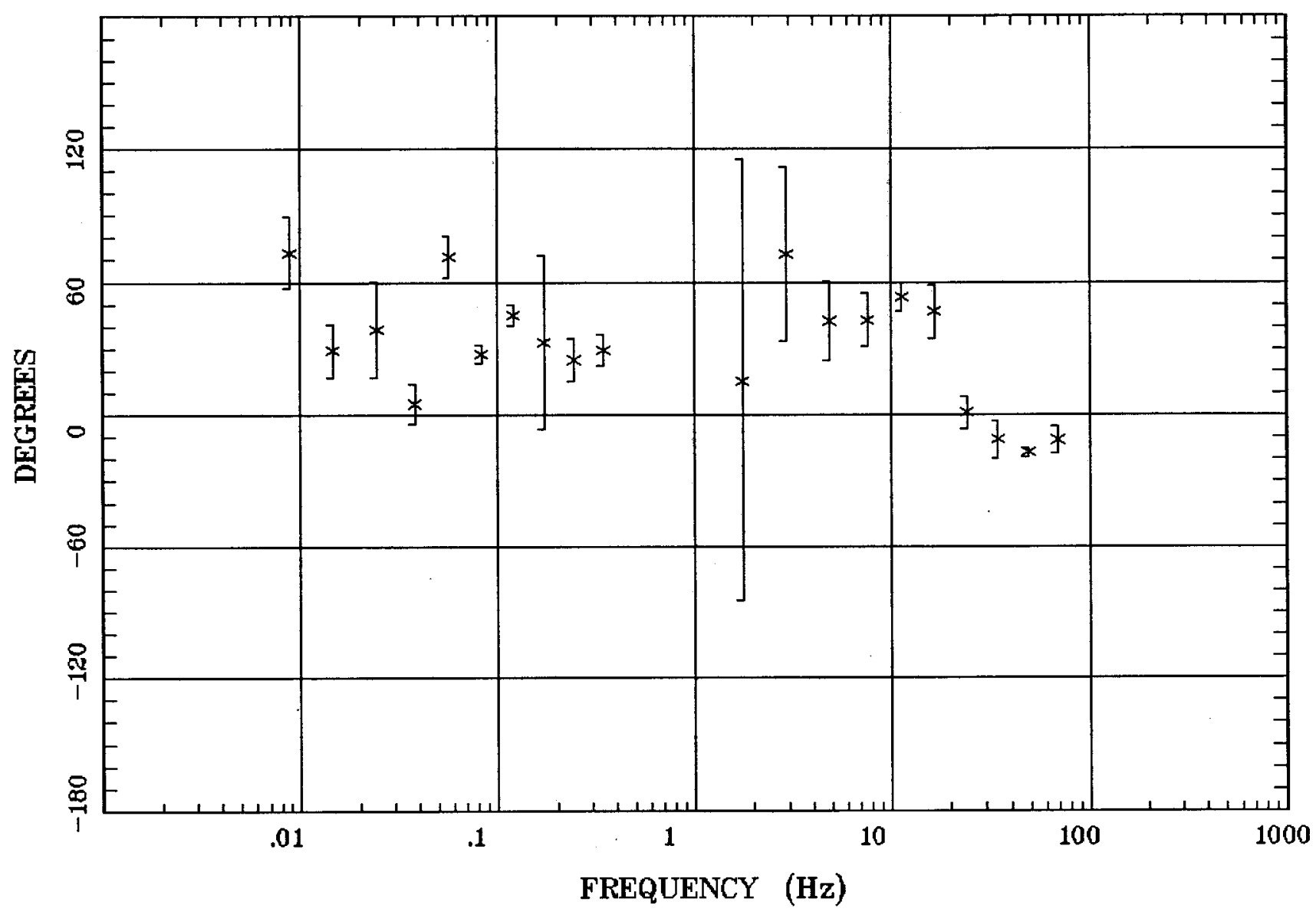

Client: Espanola Basin

Remote: none

Acquired: 11:2 Jul 19, 2004 Survey Co:USGS
Rotation:

Filename: rr23m.avg

Channels: Ch1 Ch2 Ch3 Ch4 Ch5 Ch3 Ch4 Plotted: 11:44 Jan 12, 2006

$<$ EMI - ElectroMagnetic Instruments 


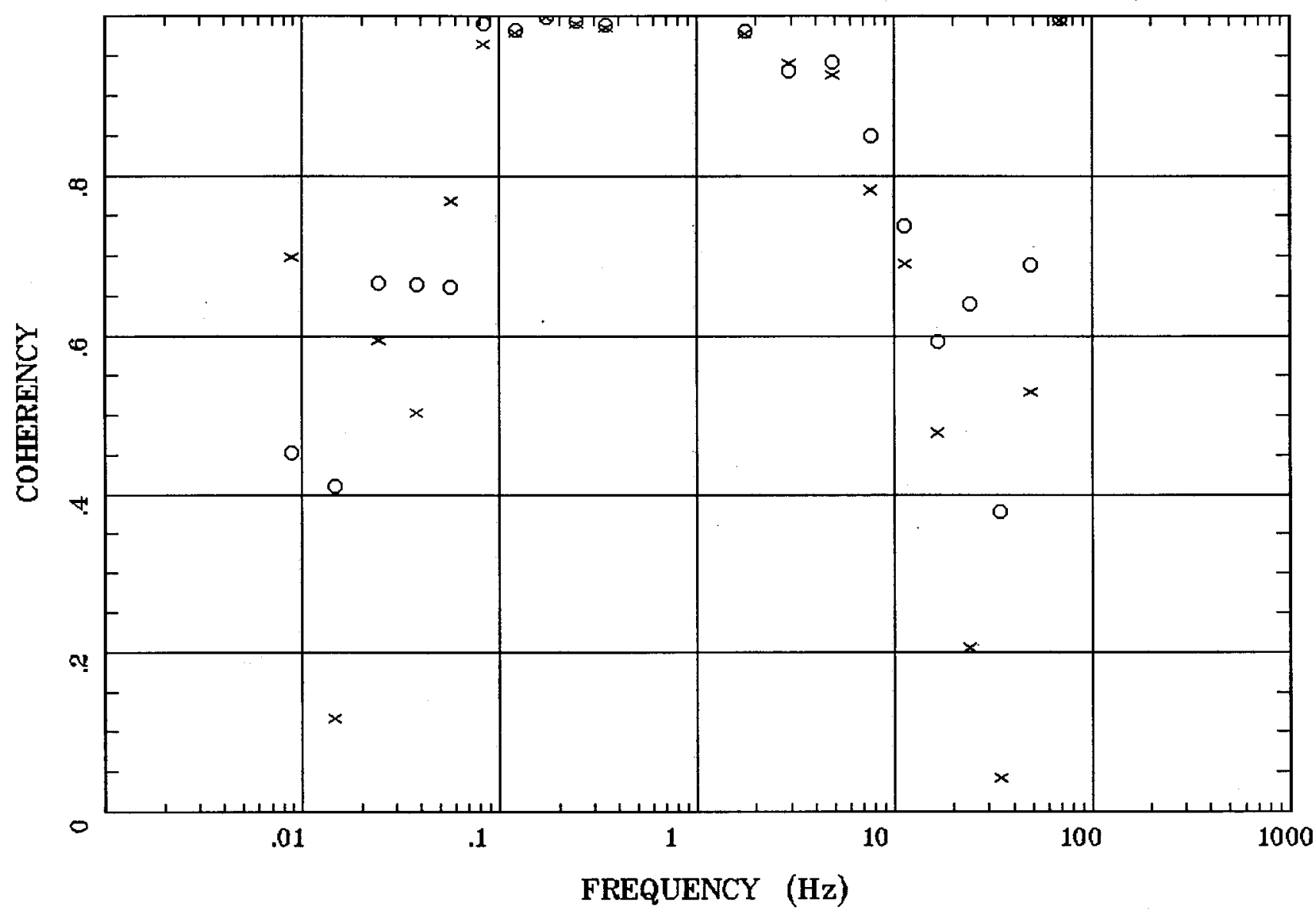

Client: Espanola Basin

Remote: none

Acquired: 11:2 Jul 19, 2004

Survey Co:USGS
Rotation:

Filename: rr23m.avg

Channels: Ch1 Ch2 Ch3 Ch4 Ch5 Ch3 Ch4

Plotted: 11:44 Jan 12, 2006

< EMI - ElectroMagnetic Instruments 


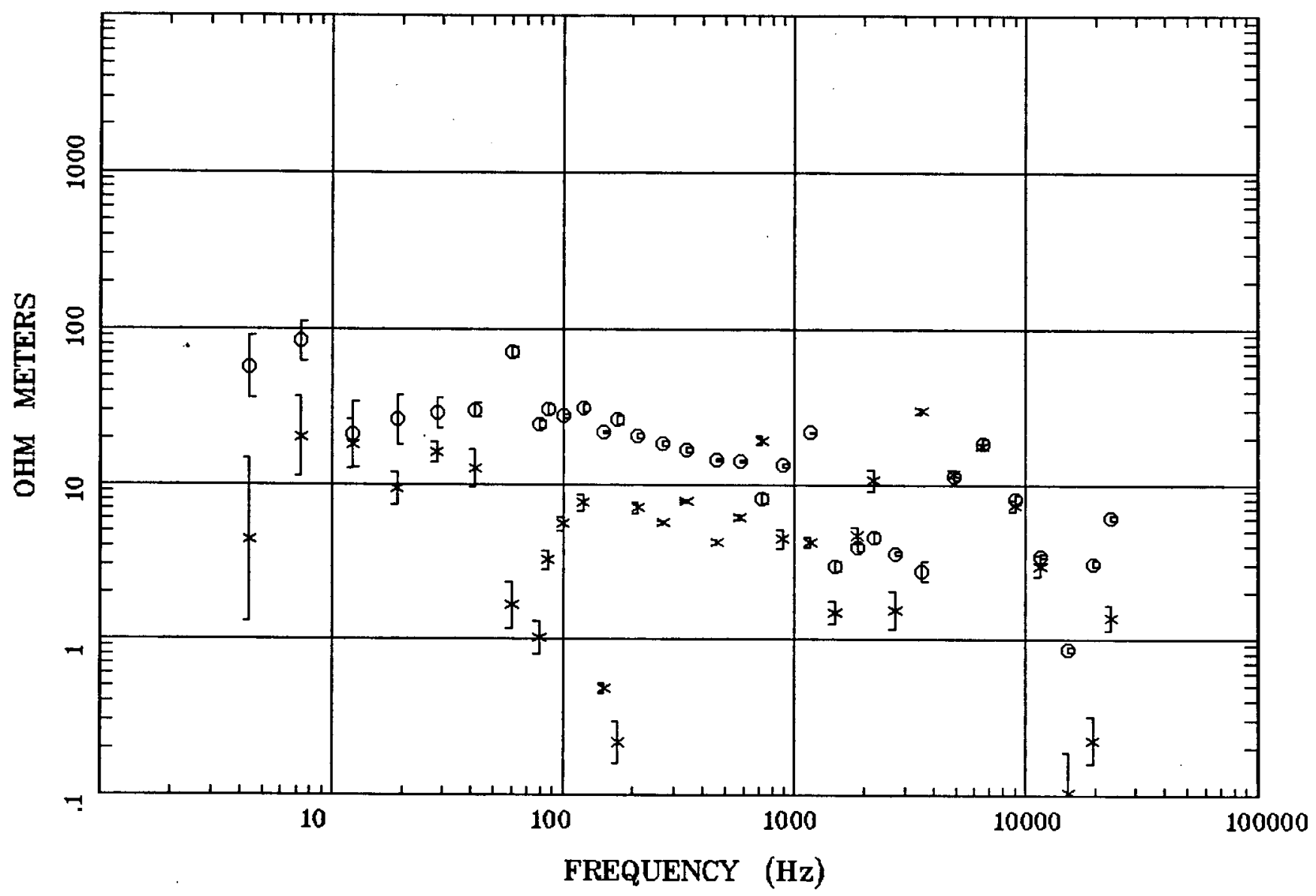

Client: Espanola Basin Remote: none Acquired: 14:4 Jul 17, 2004 Survey Co:USGS
Rotation:

Filename: rr20a.avg

Channels: Ch1 Ch2 Ch3 Ch4 Ch5 Ch3 ch4 Plotted: 09:46 Jan 10, 2006

< EMI - ElectroMagnetic Instruments > 


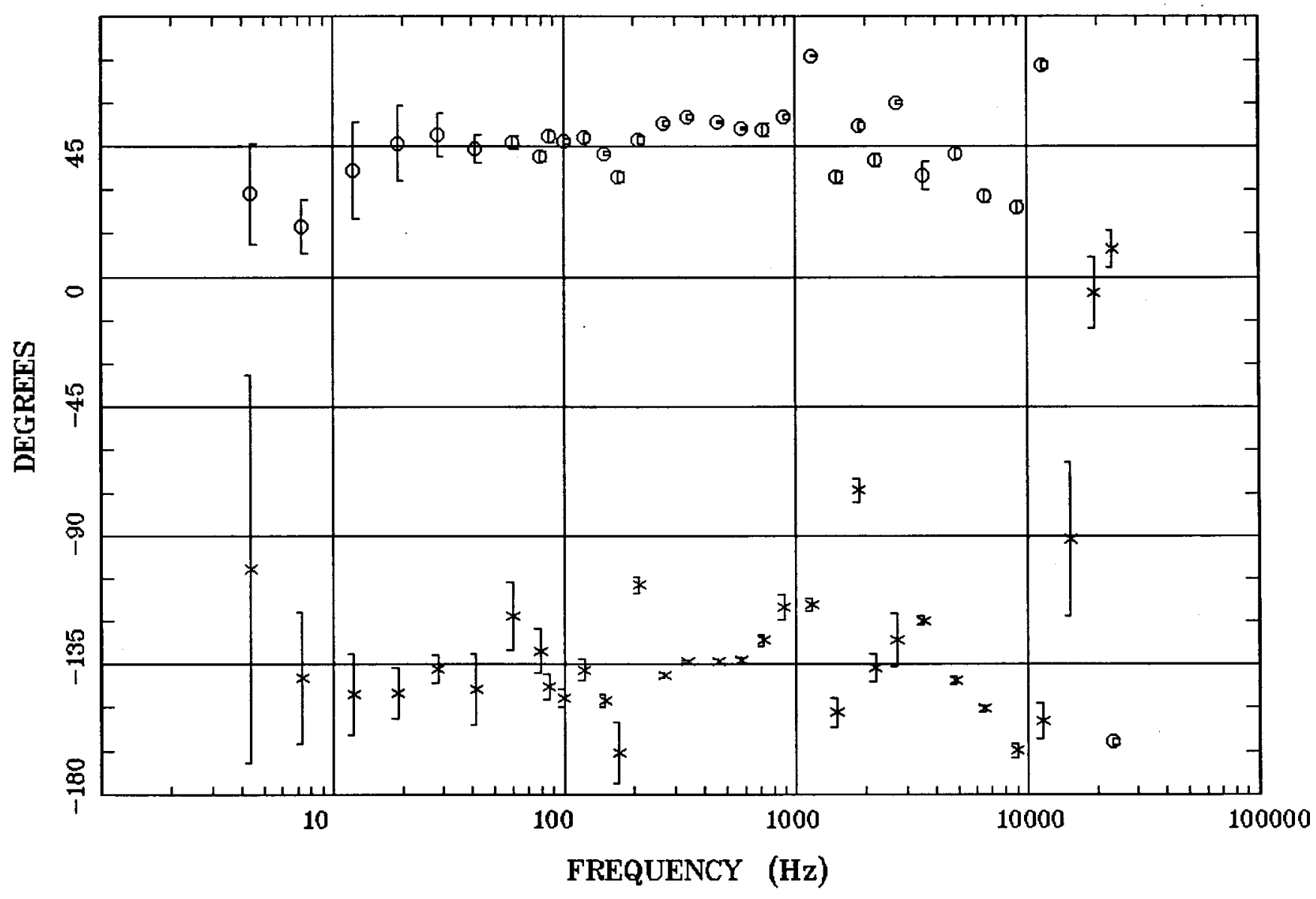

Client: Espanola Basin Remote: none Acquired: 14:4 Jul 17, 2004 Survey Co:USGS
Rotation:

Filename: rr20a.avg

Channels: Ch1 Ch2 Ch3 Ch4 Ch5 Ch3 Ch4 Plotted: 09:46 Jan 10, 2006

$<$ EMI - ElectroMagnetic Instruments 


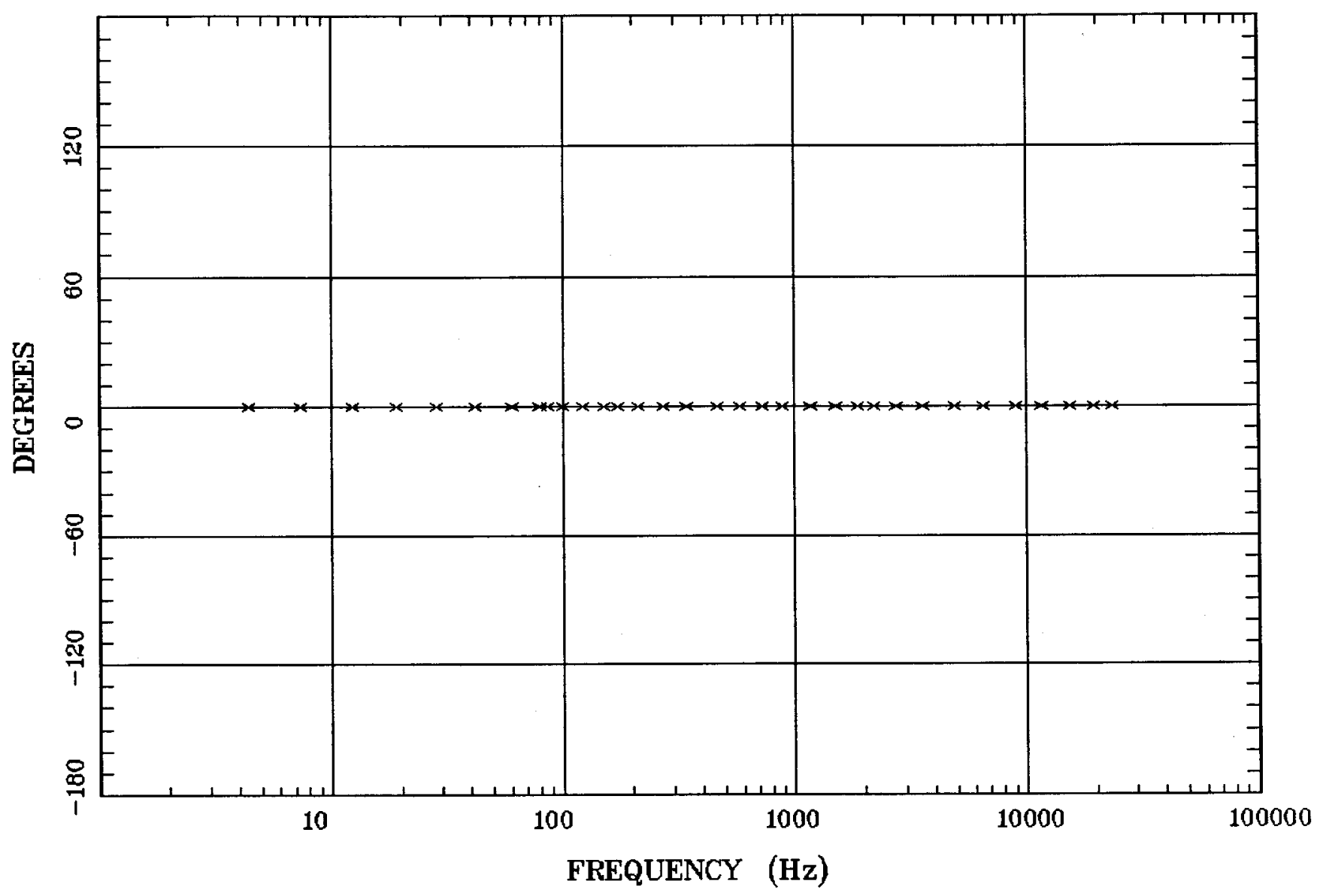

Client: Espanola Basin

Remote: none

Acquired: 14:4 Jul 17, 2004

Survey Co:USGS
Rotation:

Filename: rr20a.avg

Channels: Ch1 Ch2 Ch3 Ch4 Ch5 Ch3 Ch4

Plotted: 09:46 Jan 10, 2006

< EMI - ElectroMagnetic Instruments 


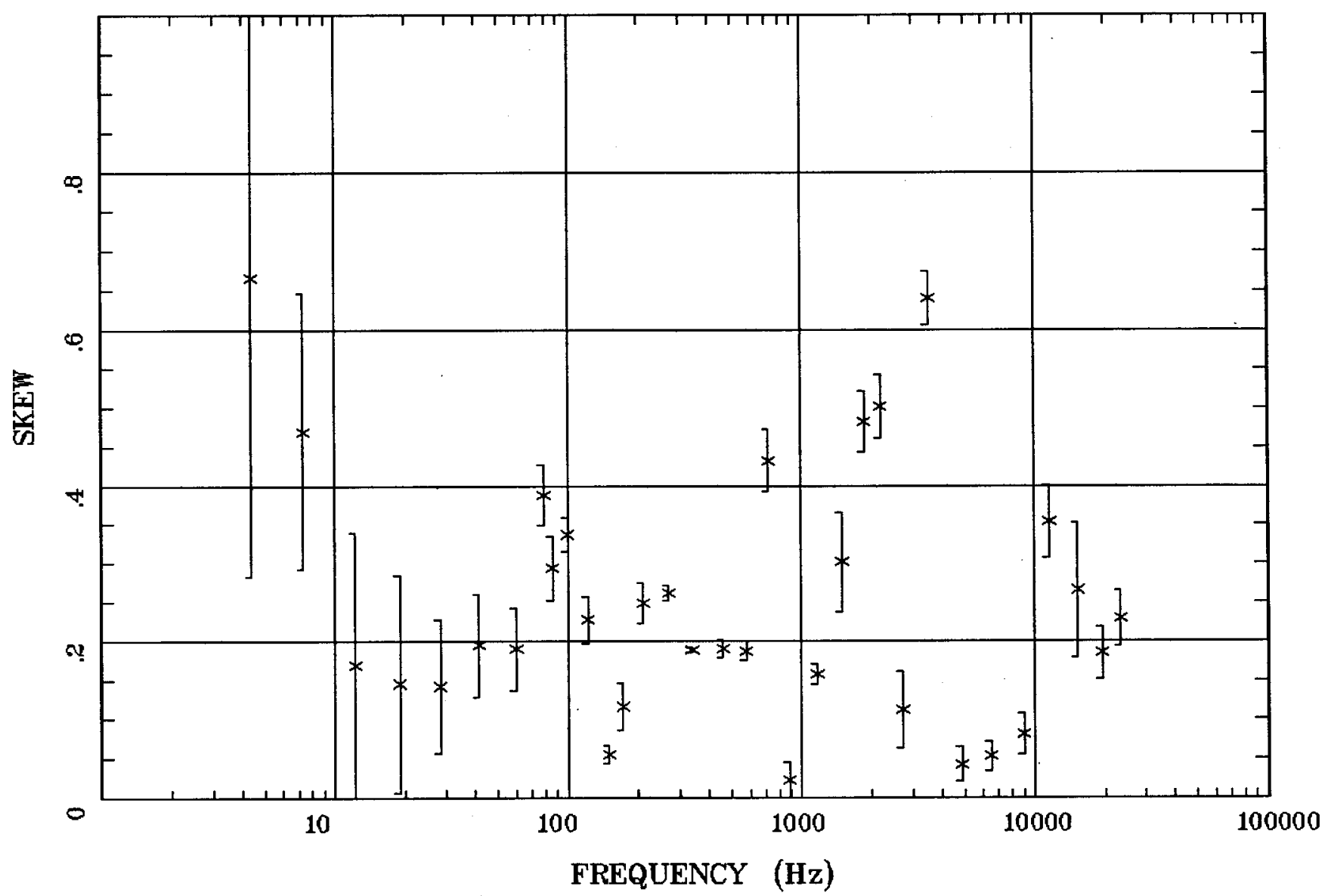

Client: Espanola Basin Remote: none Acquired: 14:4 Jul 17, 2004 Survey Co:USGS
Rotation:

Filename: rr20a.avg

Channels: Ch1 Ch2 Ch3 Ch4 Ch5 Ch3 Ch4 Plotted: 09:46 Jan 10, 2006

< EMI - ElectroMagnetic Instruments > 


\section{E MULT Coh.}

Albuquerque $100 \mathrm{~K}$

Station RR20

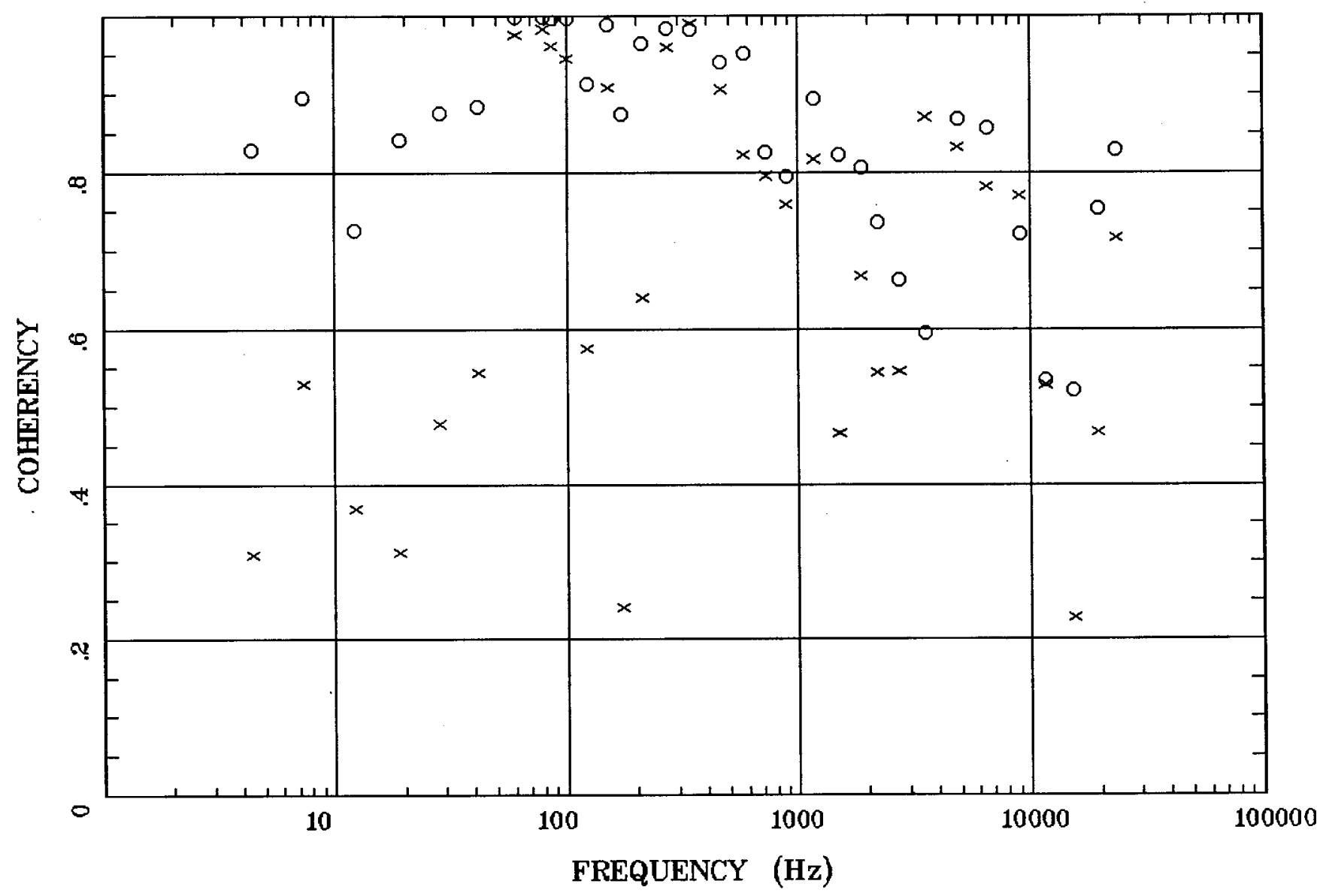

Client: Espanola Basin

Remote: none

Acquired: 14:4 Jul 17, 2004

Survey Co:USGS
Rotation:

Filename: rrz0a.avg

Channels: Ch1 Ch2 Ch3 Ch4 Ch5 Ch3 Ch4 Plotted: 09:46 Jan 10, 2006

< EMI - ElectroMagnetic Instruments 


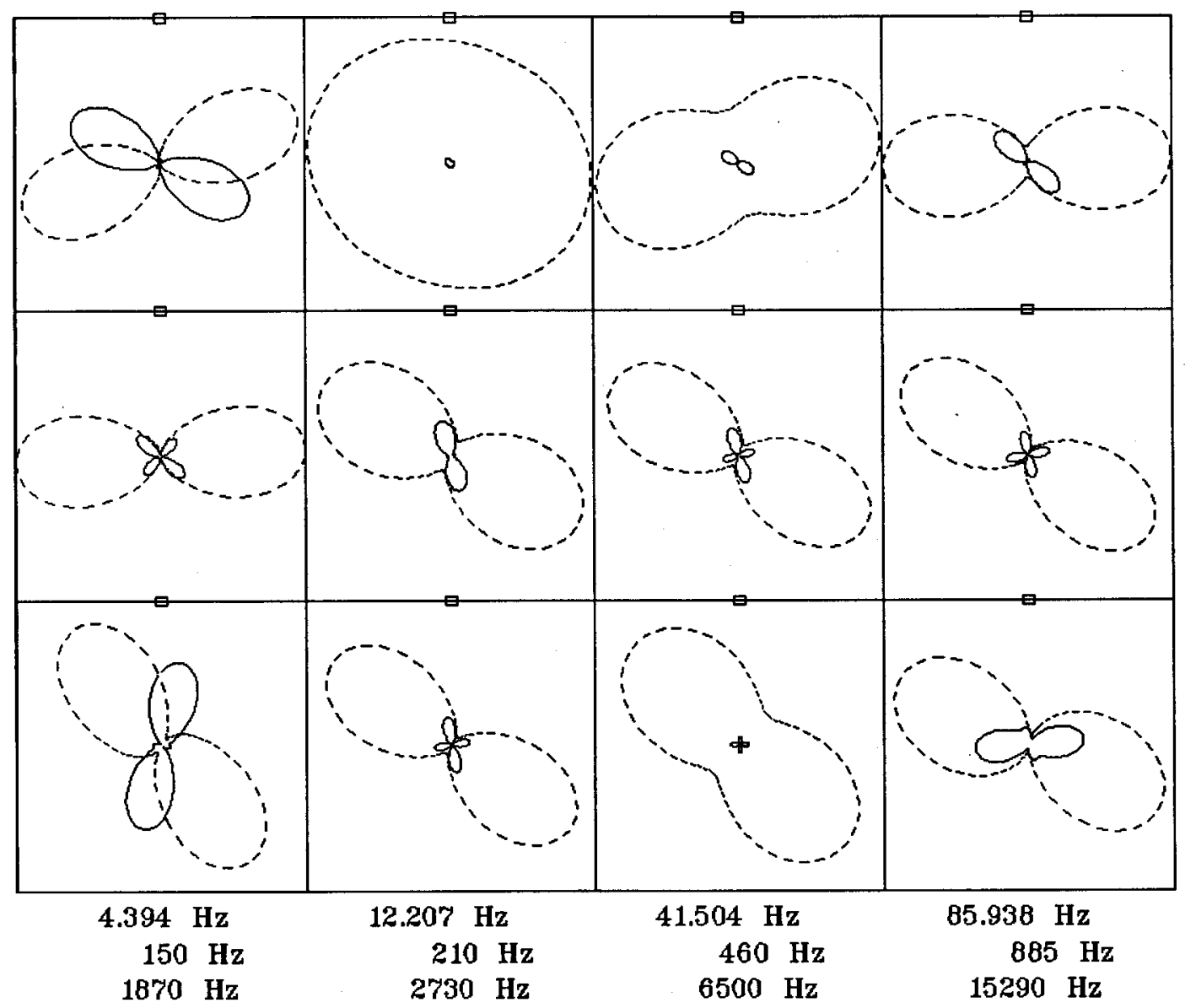

Client: Espanola Basin

Remote: none

Acquired: 14:4 Jul 17, 2004

Survey Co:USGS
Rotation:

Filename: rr20a.avg

Channels: Ch1 Ch2 Ch3 Ch4 Ch5 Ch3 Ch4

Plotted: 09:46 Jan 10, 2006

< EMI - ElectroMagnetic Instruments > 


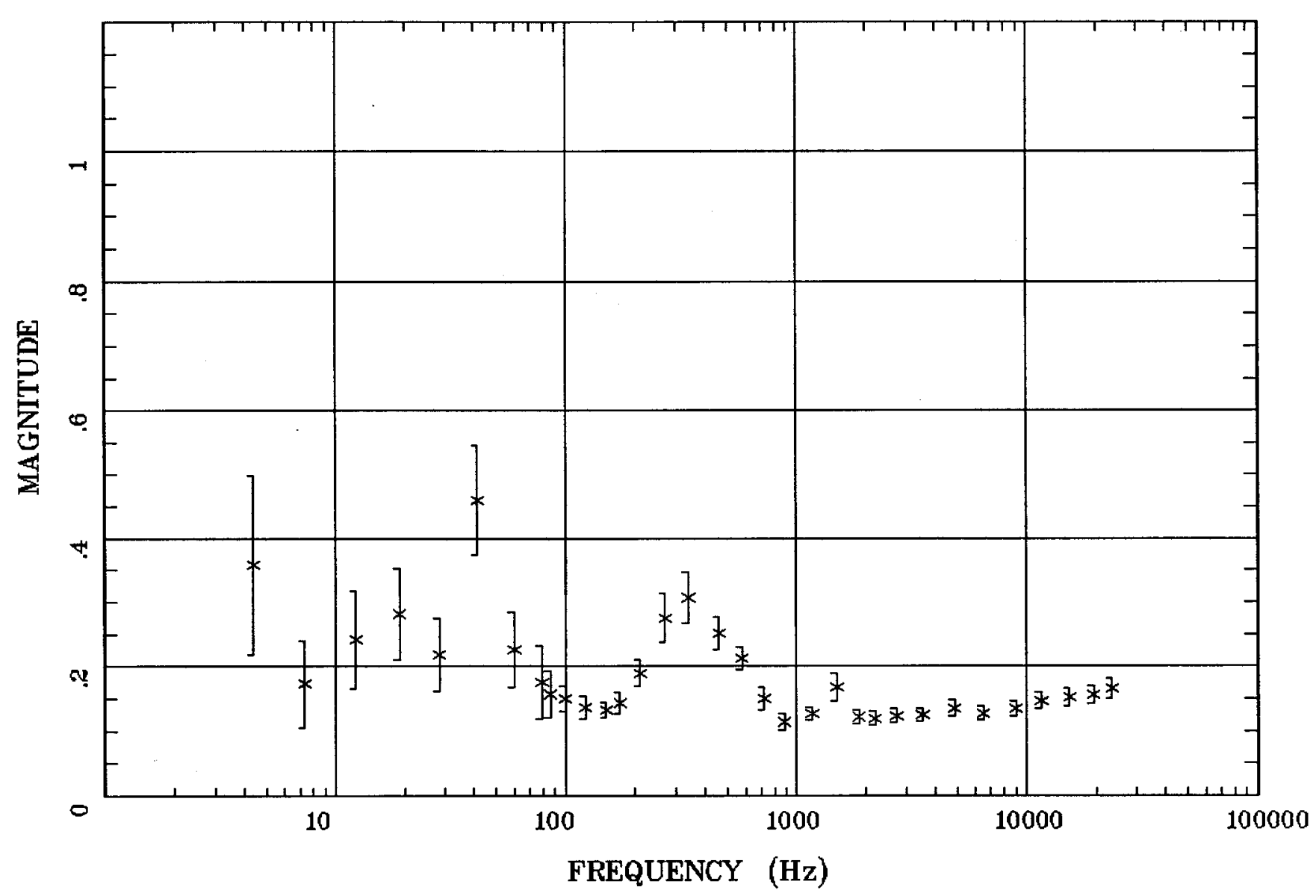

Client: Espanola Basin

Remote: none

Acquired: 14:4 Jul 17, 2004

Survey Co:USGS
Rotation:

Filename: rr20a.avg

Channels: Ch1 Ch2 Ch3 Ch4 Ch5 Ch3 Ch4

Plotted: 09:46 Jan 10, 2006

< EMI - ElectroMagnetic Instruments > 




Client: Espanola Basin

Remote: none

Acquired: 14:4 Jul 17, 2004

Survey Co:USGS
Rotation:

Filename: rr20a.avg

Channels: Ch1 Ch2 Ch3 Ch4 Ch5 Ch3 Ch4

Plotted: 09:47 Jan 10, 2006

< EMI - ElectroMagnetic Instruments > 


\section{HzHx.x Coh HzHy.o}

Albuquerque $100 \mathrm{~K}$

Station RR20

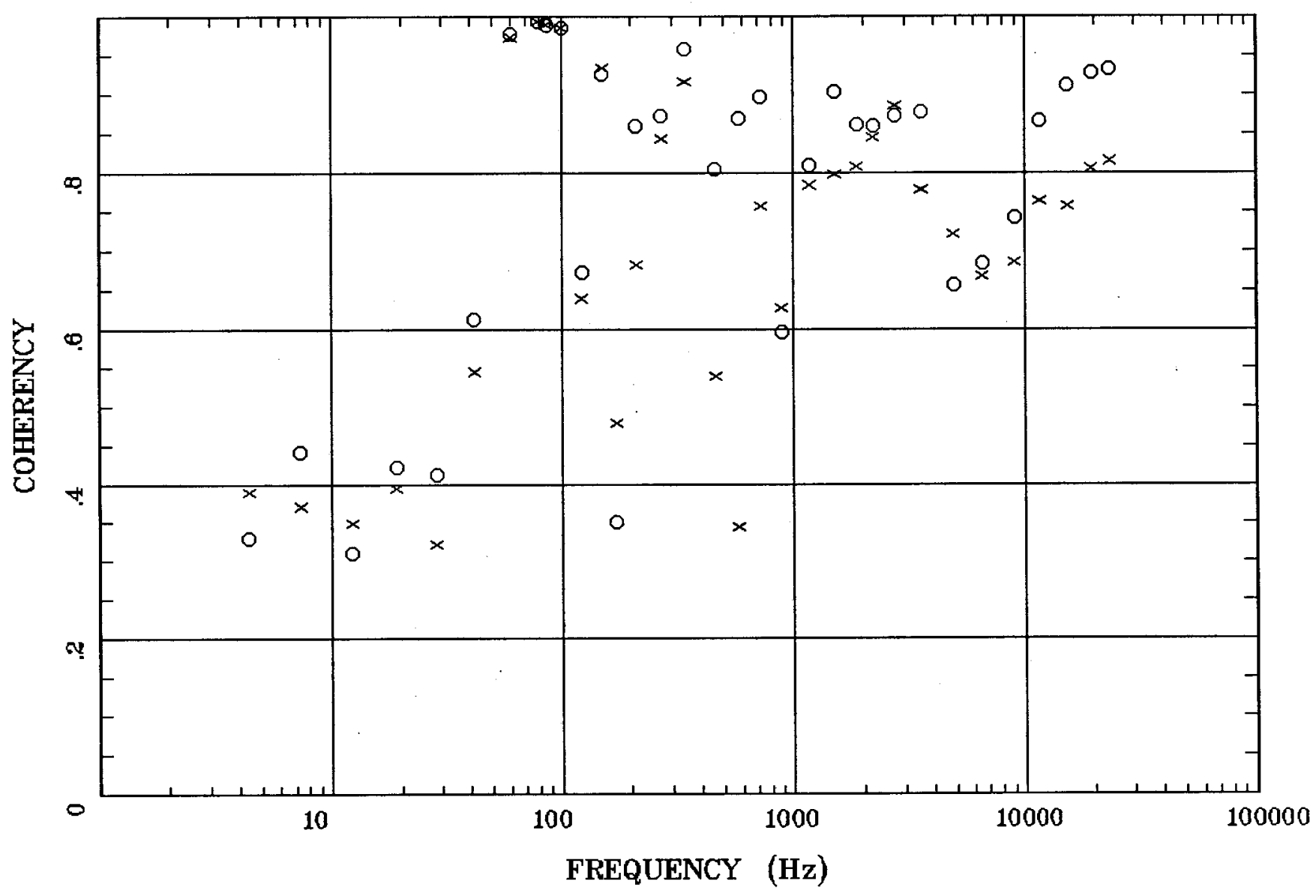

Client: Espanola Basin

Remote: none

Acquired: 14:4 Jul 17, 2004 Survey Co:USGS
Rotation:

Filename: rr20a.avg

Channels: Ch1 Ch2 Ch3 Ch4 Ch5 Ch3 Ch4

Plotted: 09:47 Jan 10, 2006

< EMI - ElectroMagnetic Instruments > 


\section{APPARENT RESISTIVITY}

Albuquerque $100 \mathrm{~K}$

Station RR21

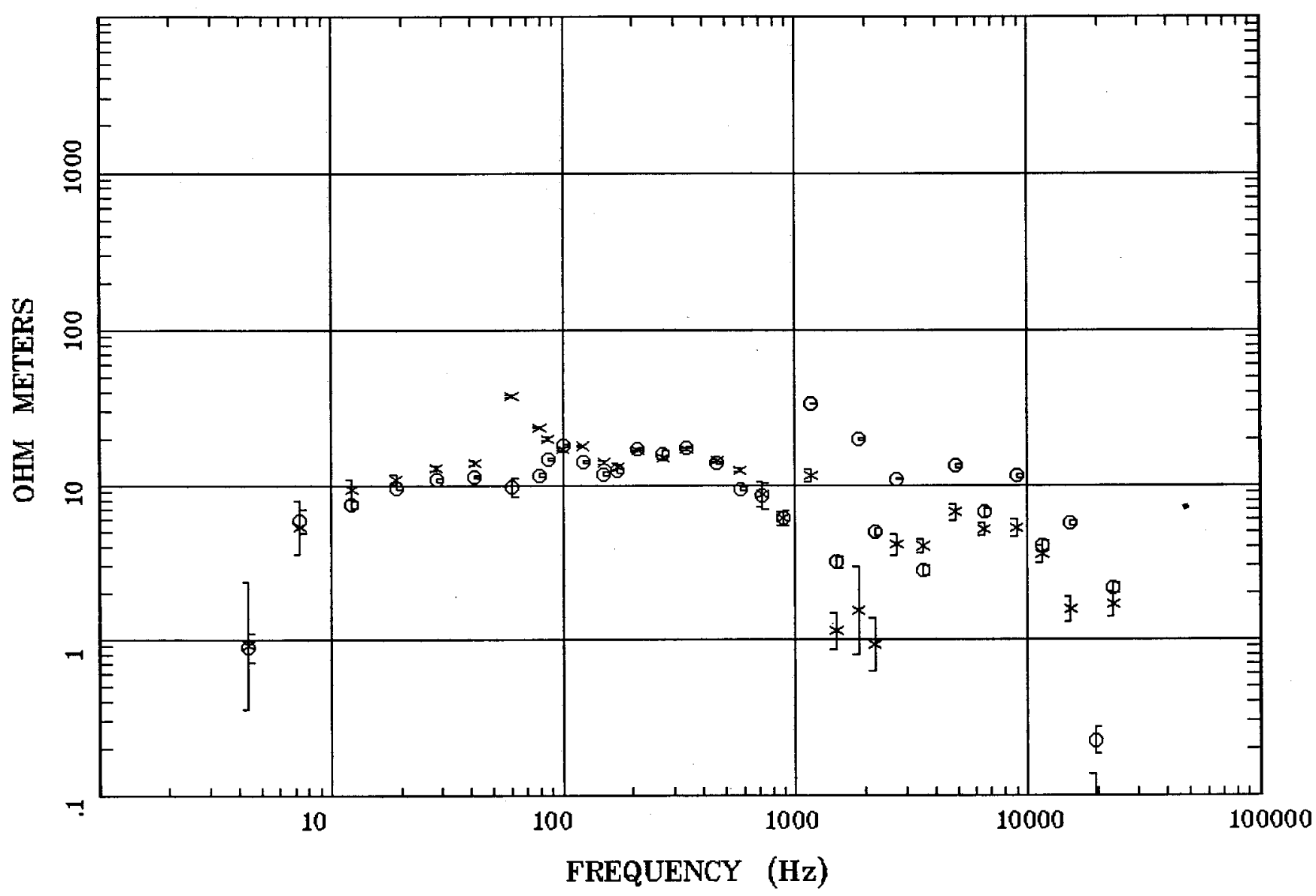

Client: Espanola Basin

Remote: none

Acquired: 12:4 Jul 18, 2004 Survey Co:USGS
Rotation:

Filename: rr21a.avg

Channels: Ch1 Ch2 Ch3 Ch4 Ch5 Ch3 Ch4 Plotted: 11:34 Jan 12, 2006

< EMI - ElectroMagnetic Instruments 


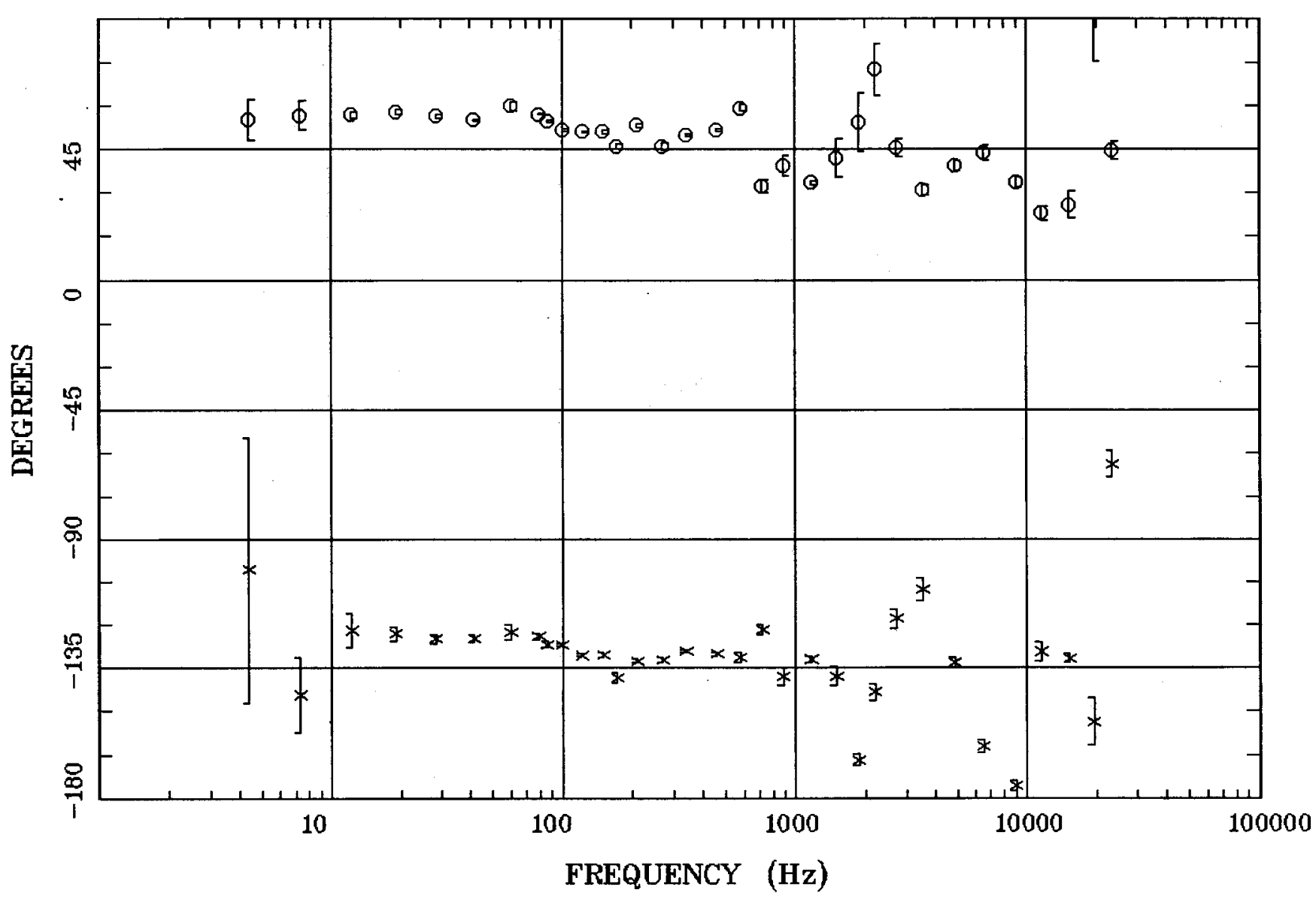

Client: Espanola Basin Remote: none

Acquired: 12:4 Jul 18, 2004 Survey Co:USGS
Rotation:

Filename: rr21a.avg

Channels: Ch1 Ch2 Ch3 Ch4 Ch5 Ch3 Ch4 Plotted: 11:34 Jan 12, 2006

$<$ EMI - ElectroMagnetic Instruments 


\section{ROTATION ANGLE}

Albuquerque $100 \mathrm{~K}$

Station RR21

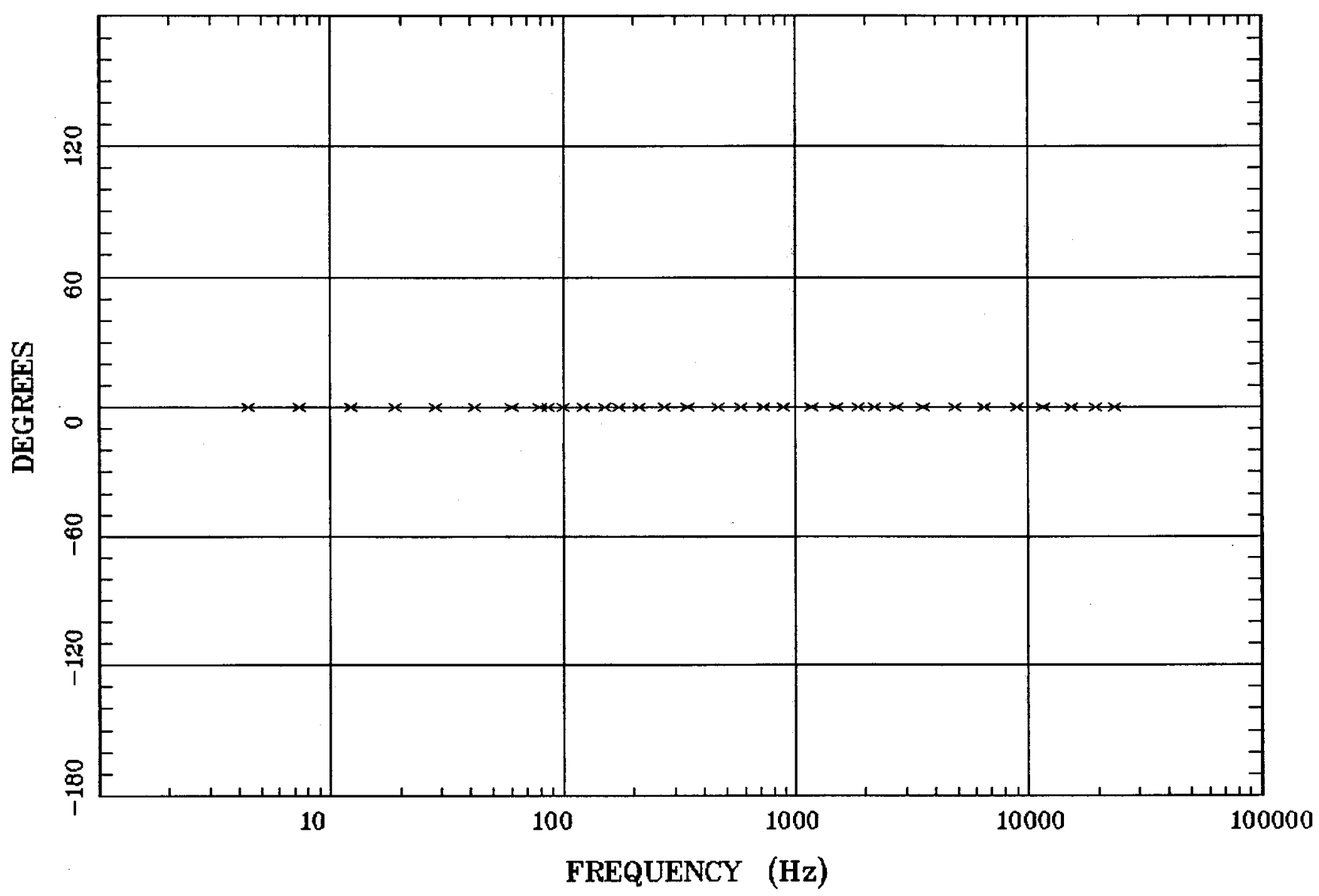

Client: Espanola Basin

Remote: none

Acquired: 12:4 Jul 18, 2004

Survey Co:USGS
Rotation:

Filename: rr21a.avg

Channels: Ch1 Ch2 Ch3 Ch4 Ch5 Ch3 Ch4

Plotted: 11:34 Jan 12, 2006

< EMI - ElectroMagnetic Instruments 


\section{IMPEDANCE SKEW}

Albuquerque $100 \mathrm{~K}$

Station RR21

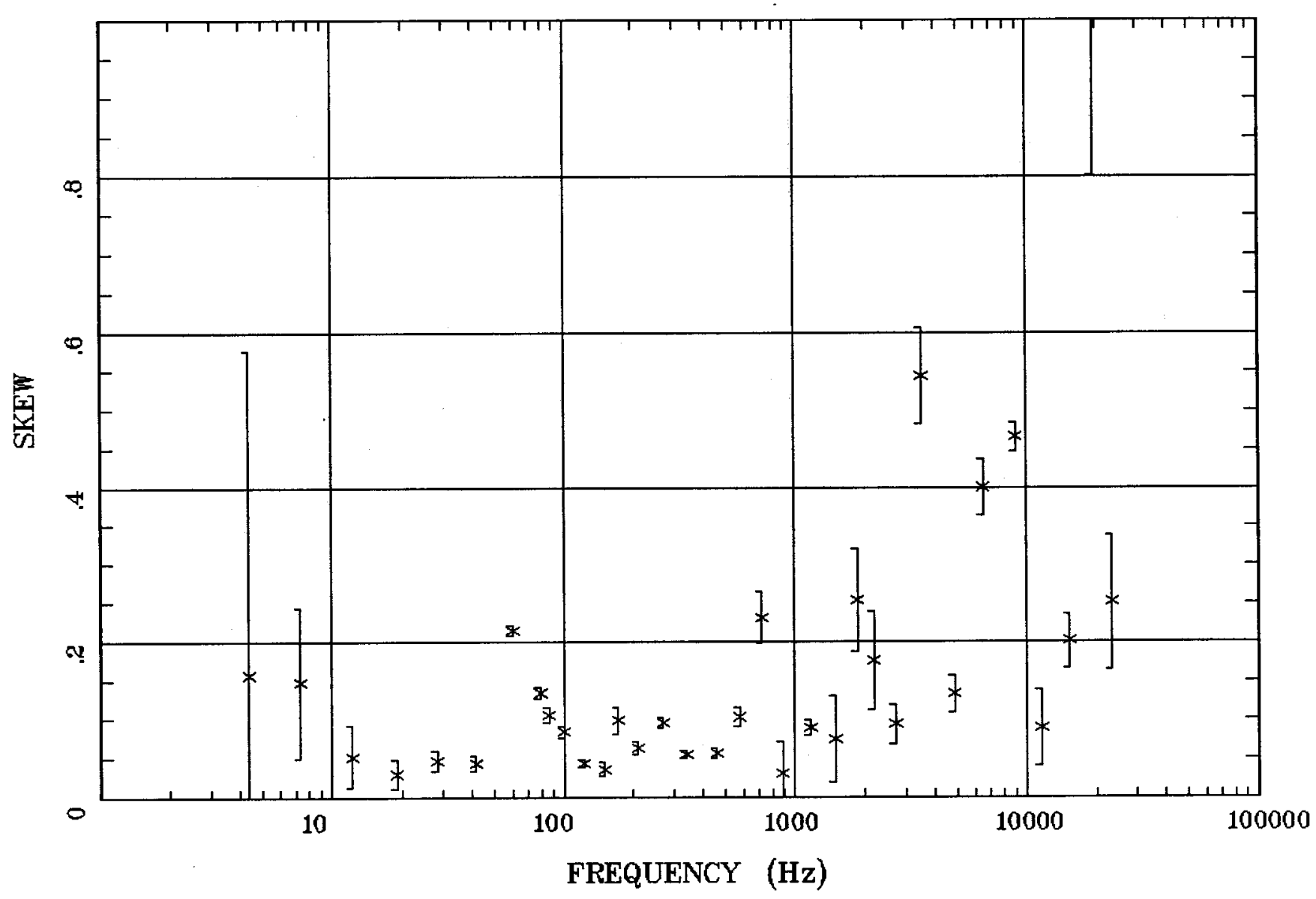

Client: Espanola Basin

Remote: none

Acquired: 12:4 Jul 18, 2004 Survey Co:USGS
Rotation:

Filename: rr21a.avg

Channels: Ch1 Ch2 Ch3 Ch4 Ch5 Ch3 Ch4

Plotted: 11:34 Jan 12, 2006

$<$ EMI - ElectroMagnetic Instruments 


\section{E MULT Coh.}

Albuquerque $100 \mathrm{~K}$

Station RR21

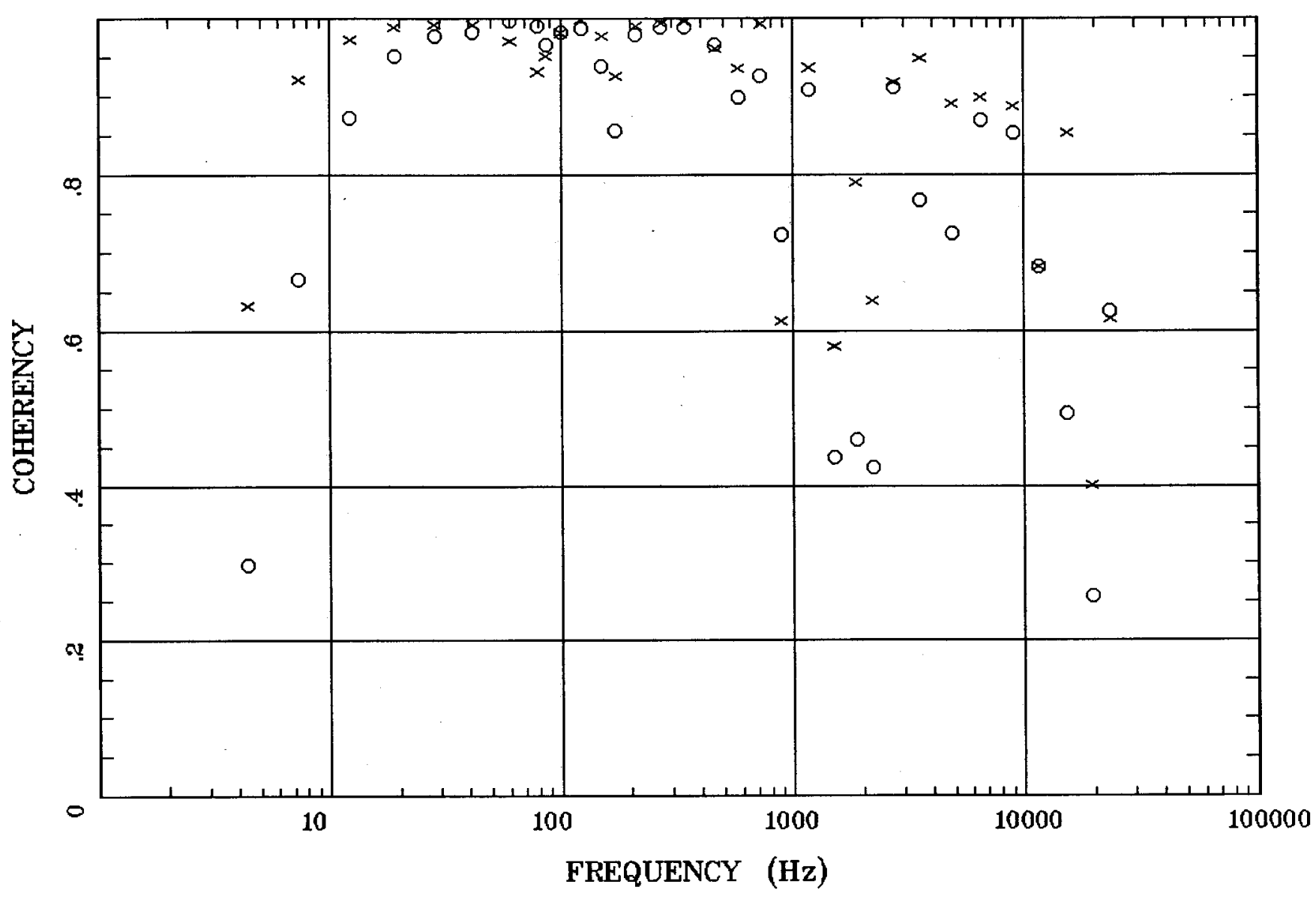

Client: Espanola Basin

Remote: none

Acquired: 12:4 Jul 18, 2004 Survey Co:USGS
Rotation:

Filename: rr21a.avg

Channels: Ch1 Ch2 Ch3 Ch4 Ch5 Ch3 Ch4 Plotted: 11:34 Jan 12, 2006

< EMI - ElectroMagnetic Instruments 


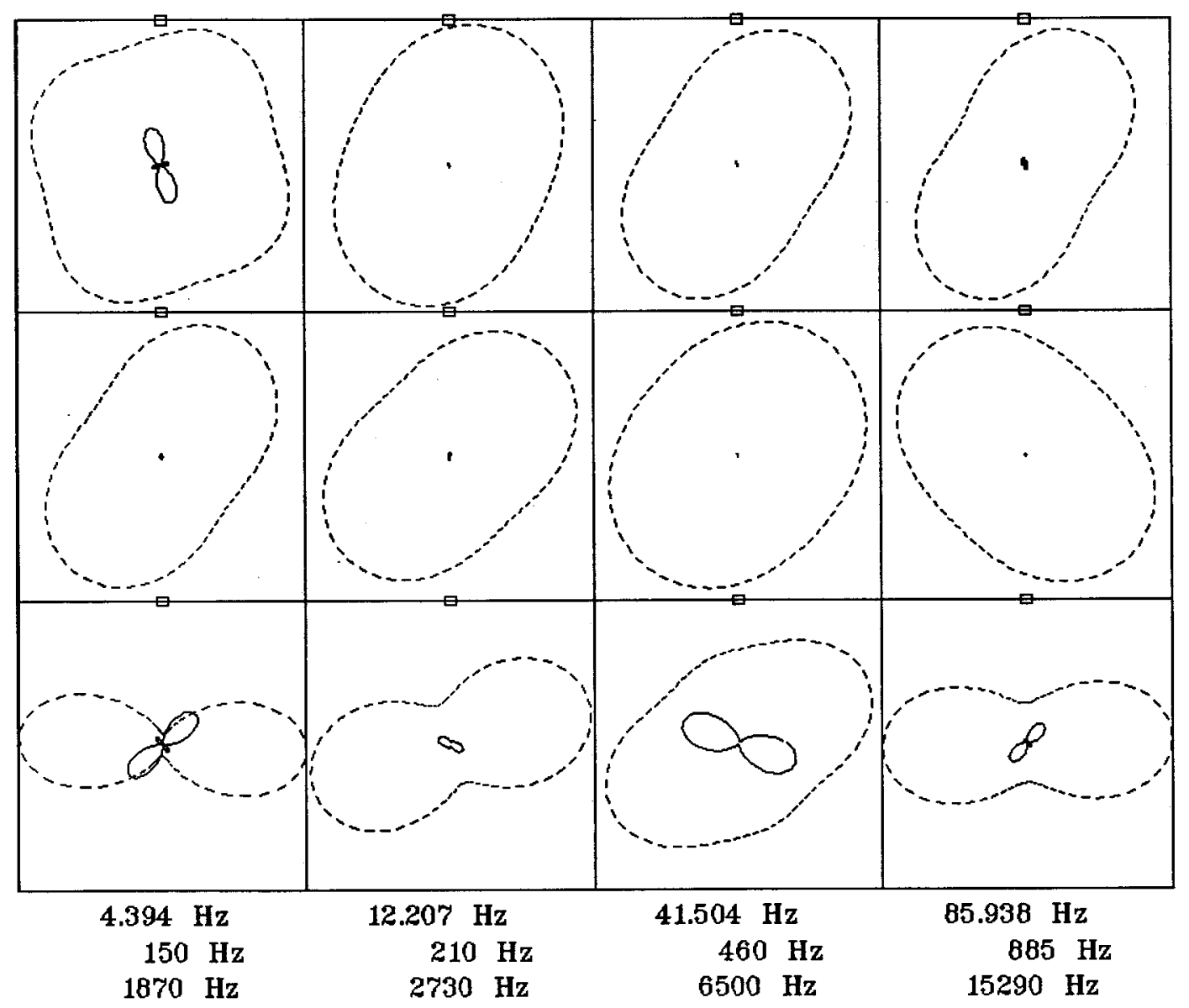

Client: Espanola Basin

Remote: none

Acquired: 12:4 Jul 18, 2004 Survey Co:USGS
Rotation:

Filename: rr21a.avg

Channels: Ch1 Ch2 Ch3 Ch4 Ch5 Ch3 Ch4

Plotted: 11:35 Jan 12, 2006

< EMI - ElectroMagnetic Instruments > 


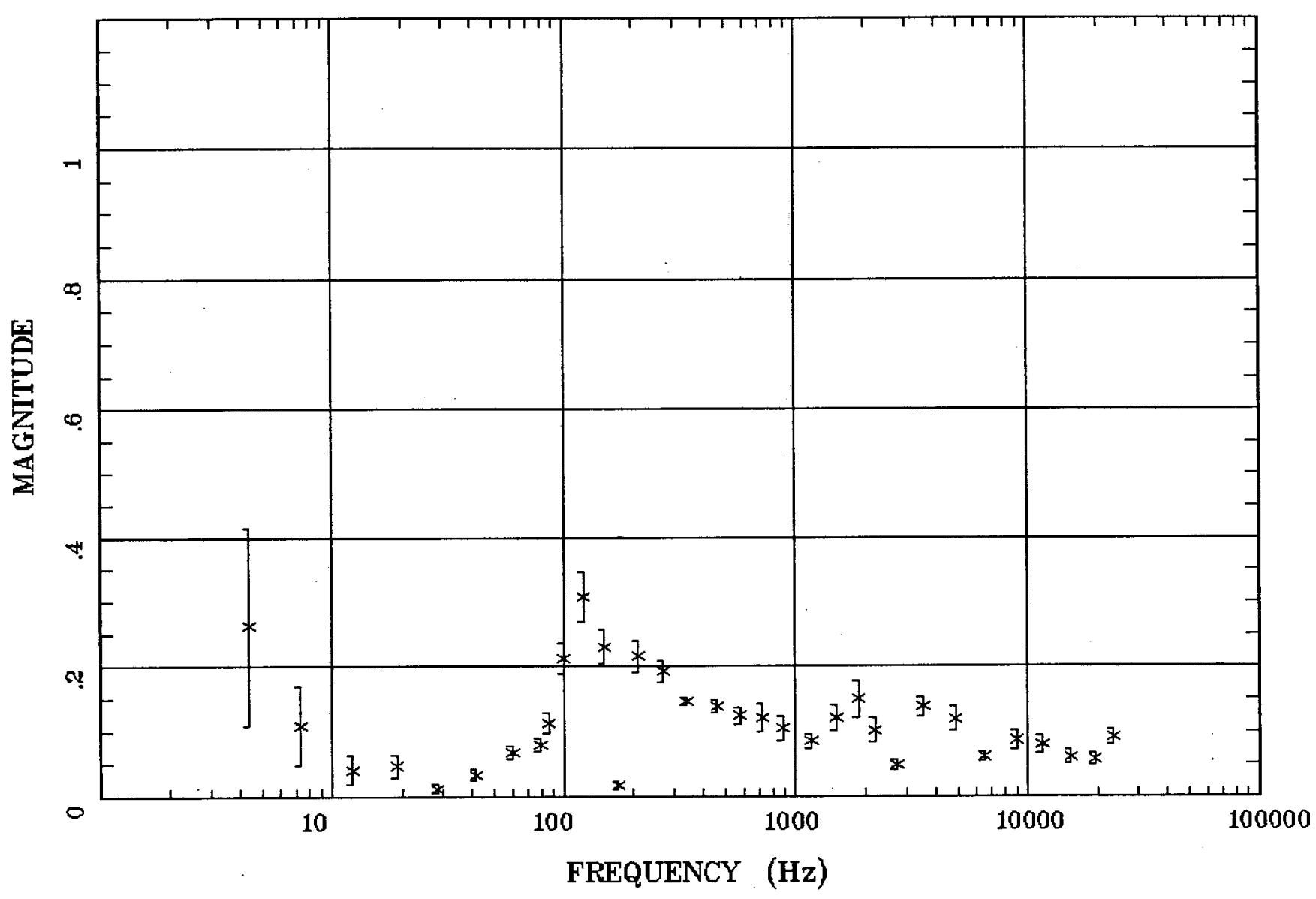

Client: Espanola Basin Remote: none Acquired: 12:4 Jul 18, 2004 Survey Co:USGS
Rotation:

Filename: rr21a.avg

Channels: Ch1 Ch2 Ch3 Ch4 Ch5 Ch3 Ch4

Plotted: 11:35 Jan 12, 2006

< EMI - ElectroMagnetic Instruments > 


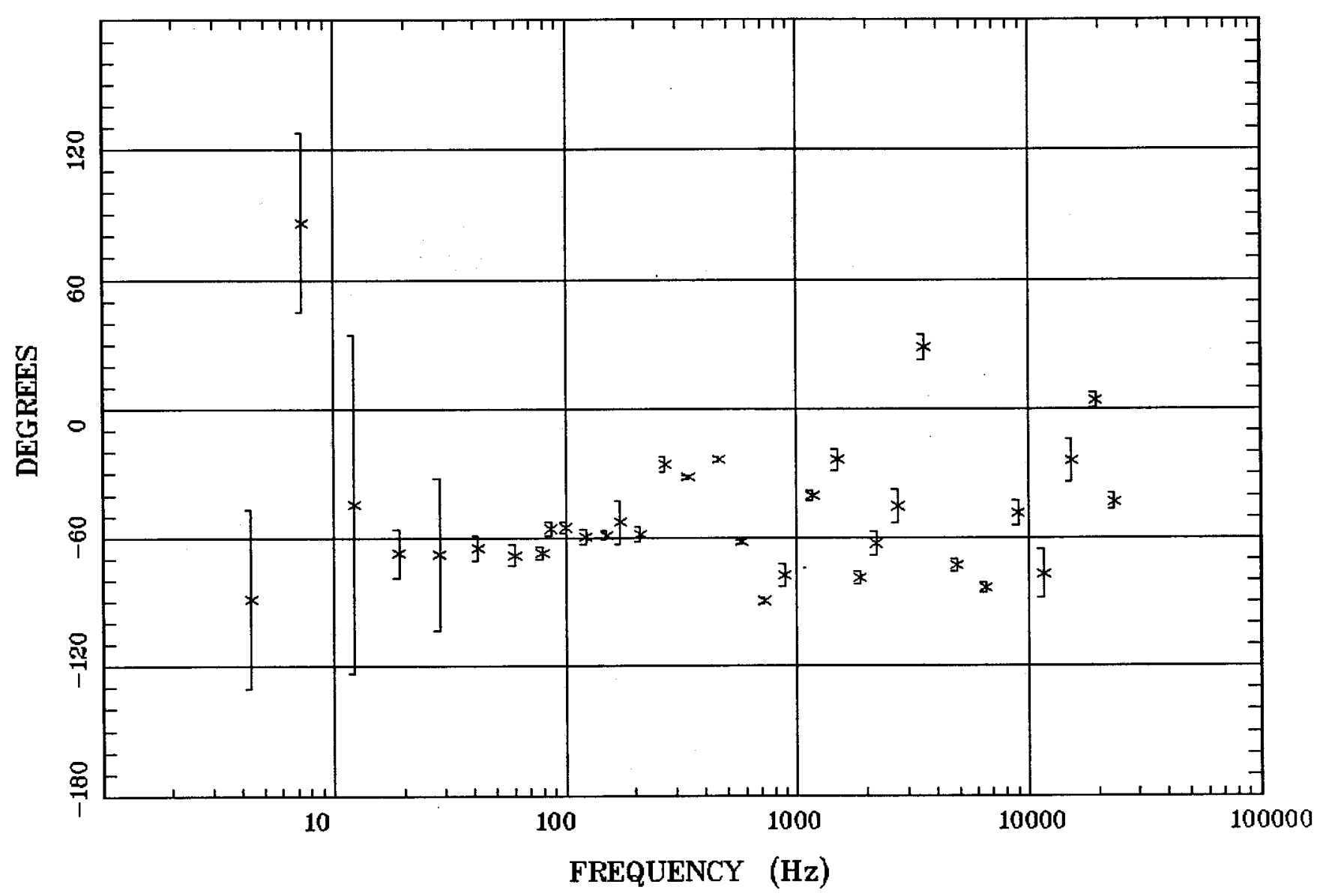

Client: Espanola Basin Remote: none Acquired: 12:4 Jul 18, 2004 Survey Co:USGS
Rotation:

Filename: rr21a.avg

Channels: Ch1 Ch2 Ch3 Ch4 Ch5 Ch3 Ch4 Plotted: 11:35 Jan 12, 2006

< EMI - ElectroMagnetic Instruments 


\section{HzHx.x Coh HzHy.o}

Albuquerque $100 \mathrm{~K}$

Station RR21

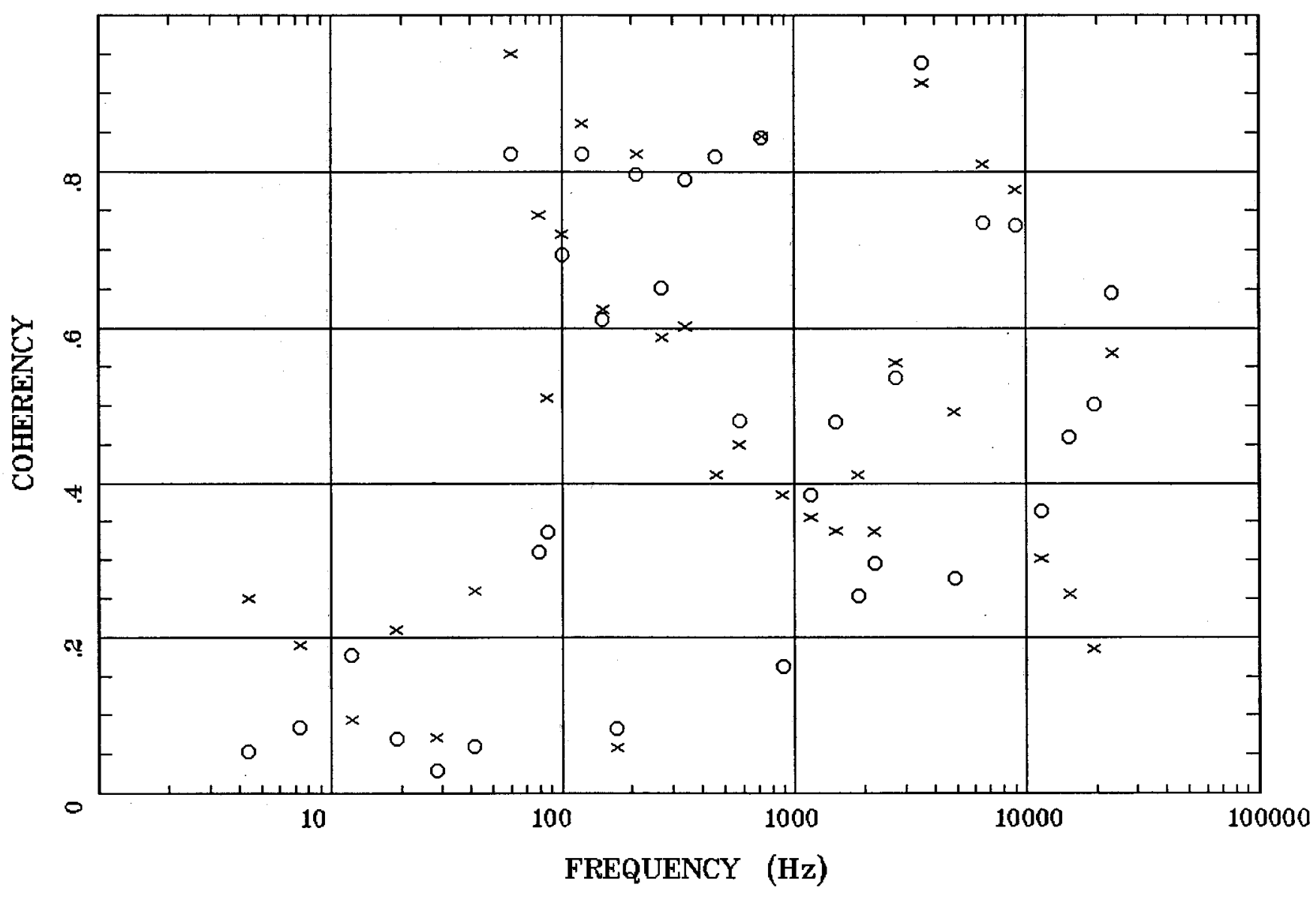

Client: Espanola Basin

Remote: none

Acquired: 12:4 Jul 18, 2004

Survey Co:USGS
Rotation:

Filename: rr21a.avg

Channels: Ch1 Ch2 Ch3 Ch4 Ch5 Ch3 Ch4

Plotted: 11:35 Jan 12, 2006

$<$ EMI - ElectroMagnetic Instruments 


\section{APPARENT RESISTIVITY}

Albuquerque $100 \mathrm{~K}$

Station RR22



Client: Espanola Basin Remote: none Acquired: 14:1 Jul 21, 2004 Survey Co:USGS
Rotation:

Filename: rr22a.avg

Channels: Ch1 Ch2 Ch3 Ch4 Ch5 Ch3 Ch4

Plotted: 11:37 Jan 12, 2006

< EMI - ElectroMagnetic Instruments > 


\section{IMPEDANCE PHASE}

Albuquerque $100 \mathrm{~K}$

Station RR22

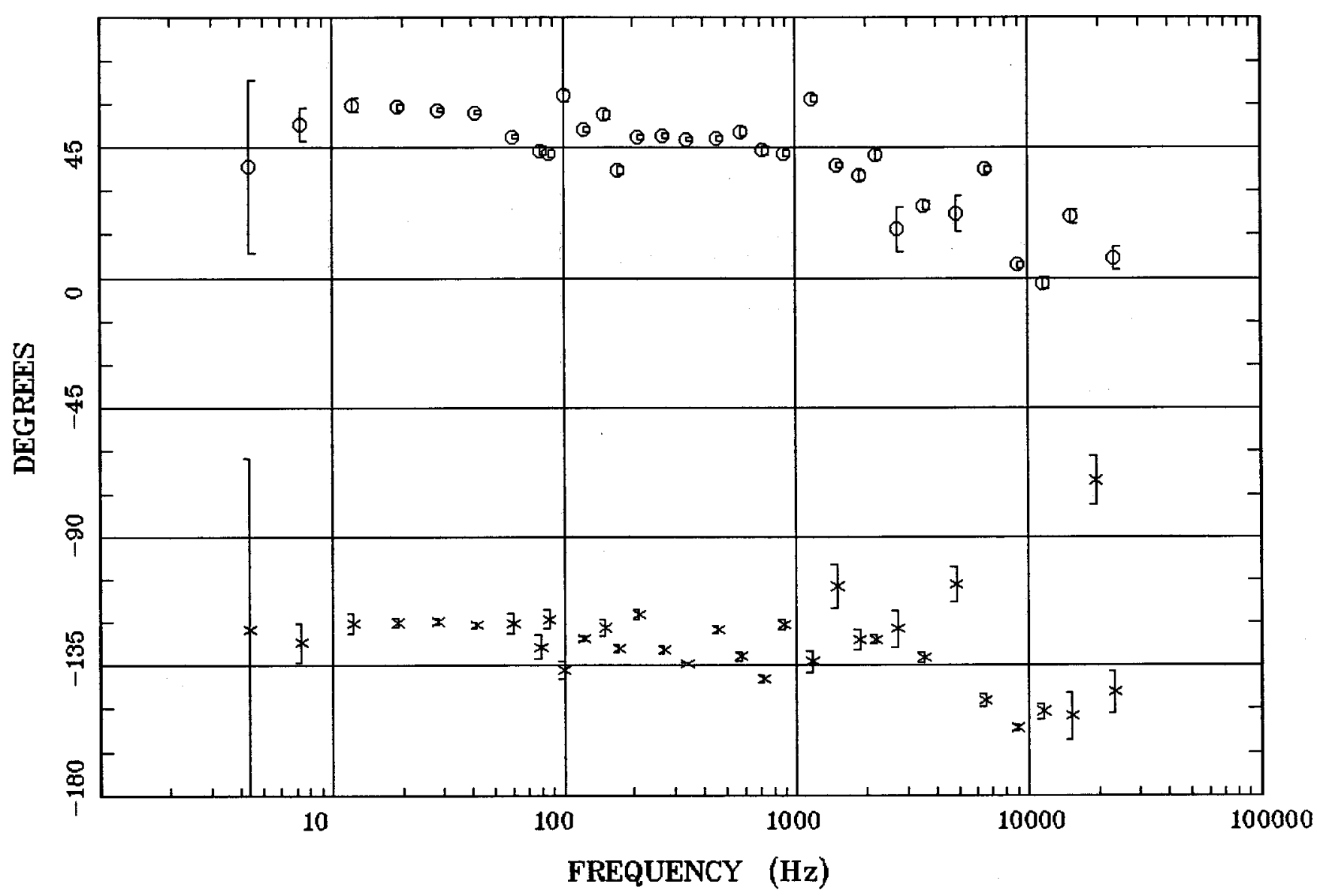

Client: Espanola Basin

Remote: none

Acquired: 14:1 Jul 21, 2004 Survey Co:USGS
Rotation:

Filename: rr22a.avg

Channels: Ch1 Ch2 Ch3 Ch4 Ch5 Ch3 Ch4

Plotted: 11:37 Jan 12, 2006

< EMI - ElectroMagnetic Instruments 







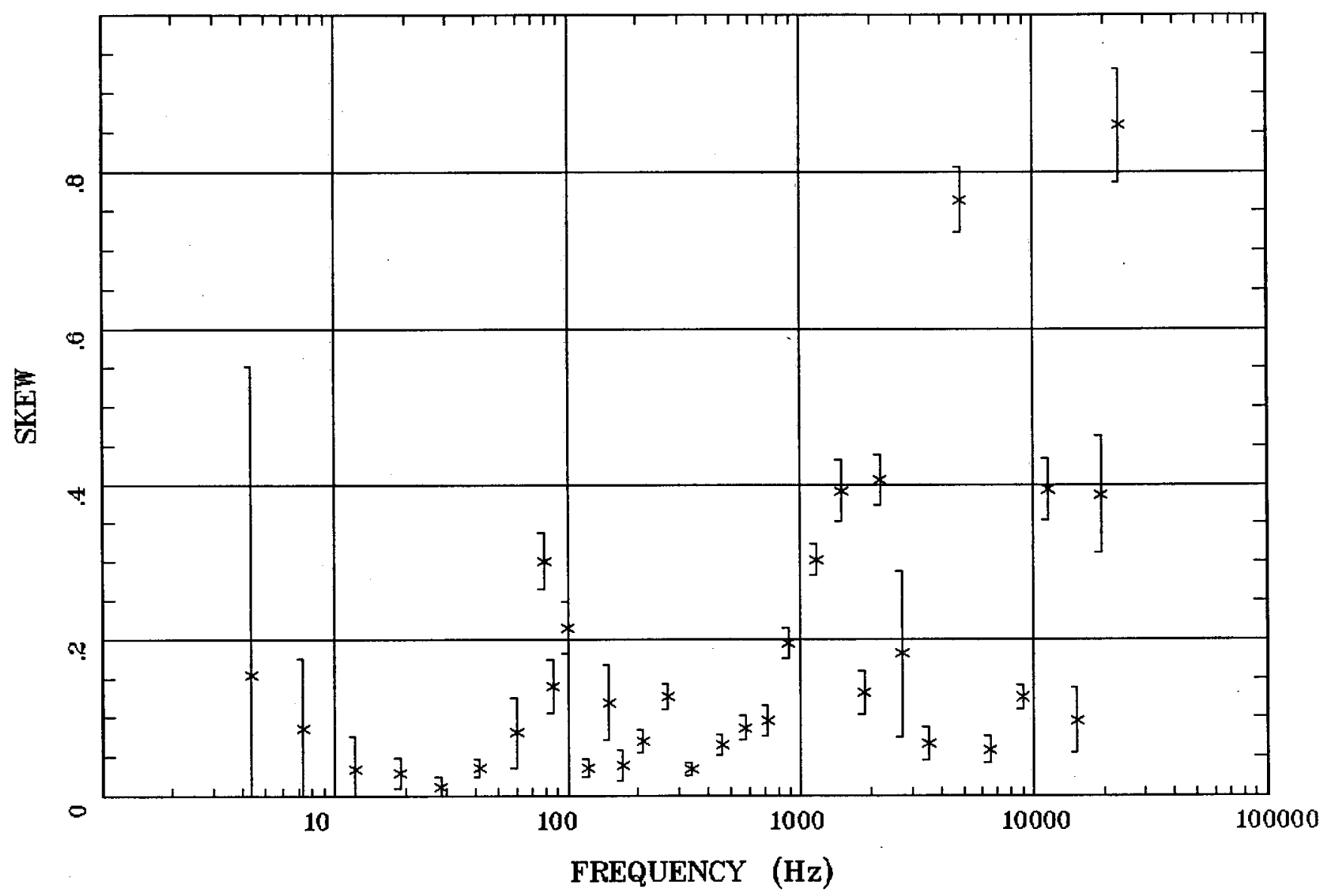

Client: Espanola Basin Remote: none

Acquired: 14:1 Jul 21, 2004

Survey Co:USGS
Rotation:

Filename: rr22a.avg

Channels: Ch1 Ch2 Ch3 Ch4 Ch5 Ch3 Ch4

Plotted: 11:37 Jan 12, 2006

< EMI - ElectroMagnetic Instruments > 


\section{E MULT Coh.}

Albuquerque $100 \mathrm{~K}$

Station RR22

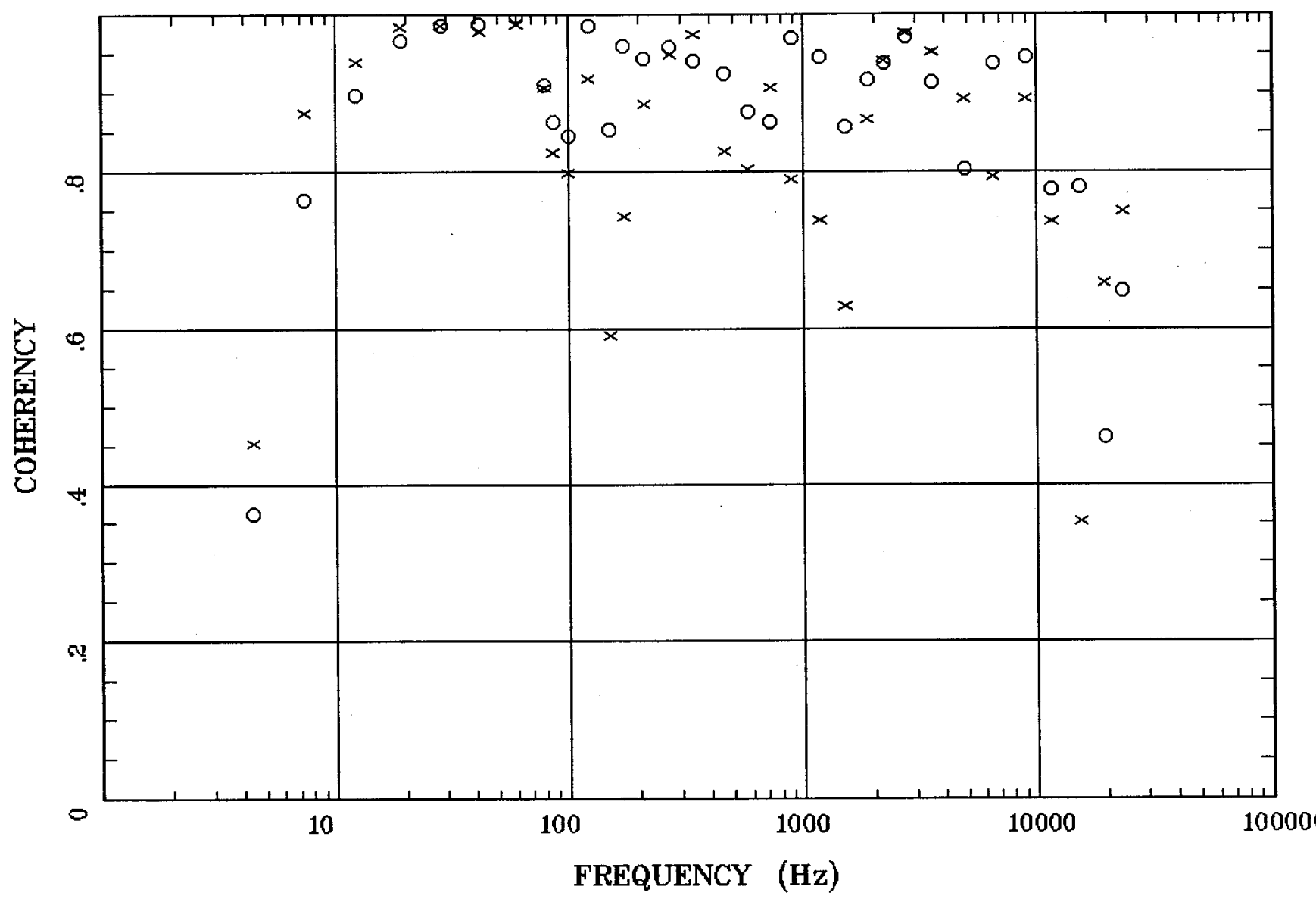

Client: Espanola Basin

Remote: none

Acquired: 14:1 Jul 21, 2004 Survey Co:USGS
Rotation:

Filename: rr22a.avg

Channels: Ch1 Ch2 Ch3 Ch4 Ch5 Ch3 Ch4 Plotted: 11:37 Jan 12, 2006

<EMI - ElectroMagnetic Instruments > 


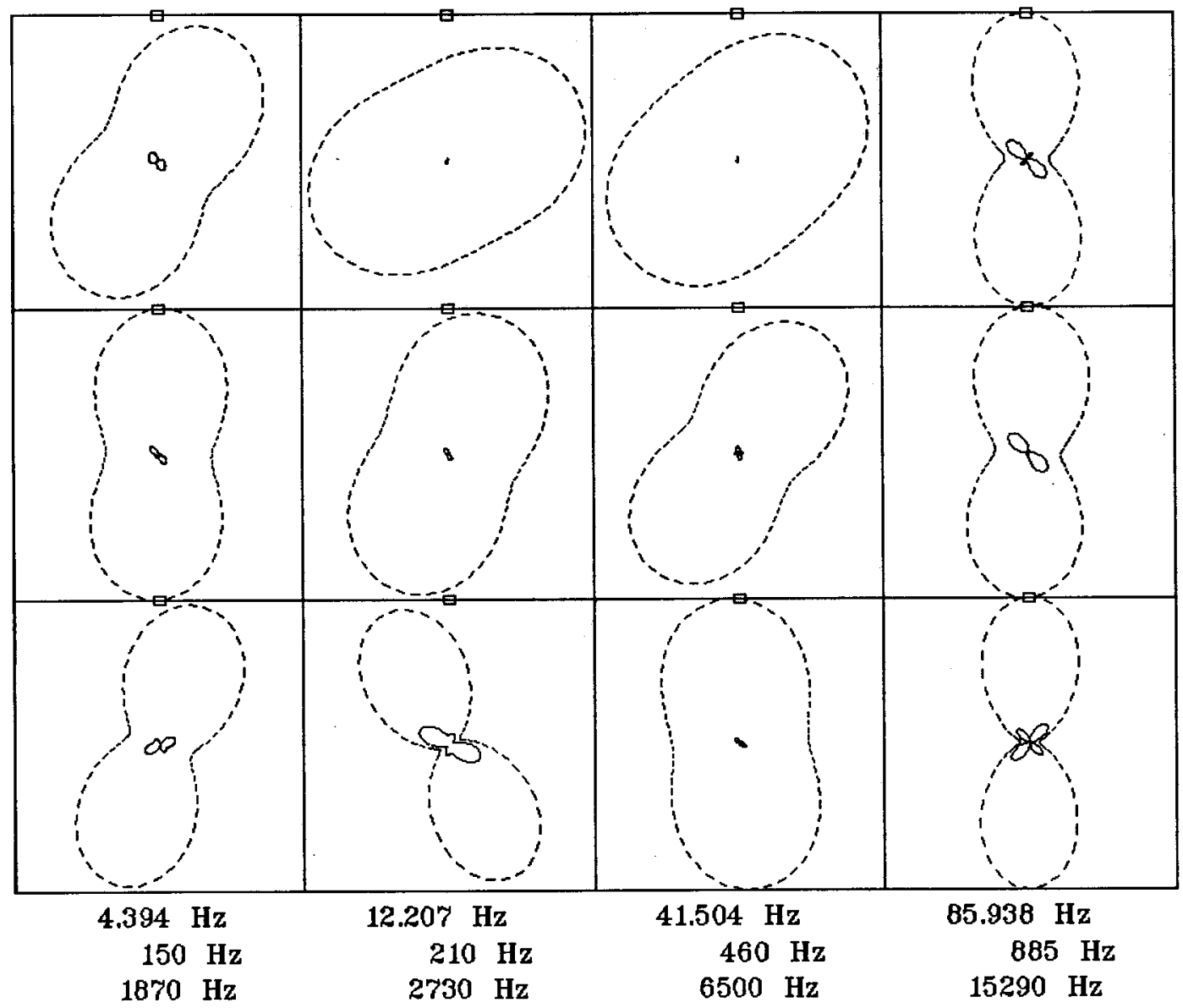

Client: Espanola Basin Remote: none Acquired: 14:1 Jul 21, 2004 Survey Co:USGS
Rotation:

Filename: rr22a.avg Channels: Ch1 Ch2 Ch3 Ch4 Ch5 Ch3 Ch4 Plotted: 11:37 Jan 12, 2006

< EMI - ElectroMagnetic Instruments 


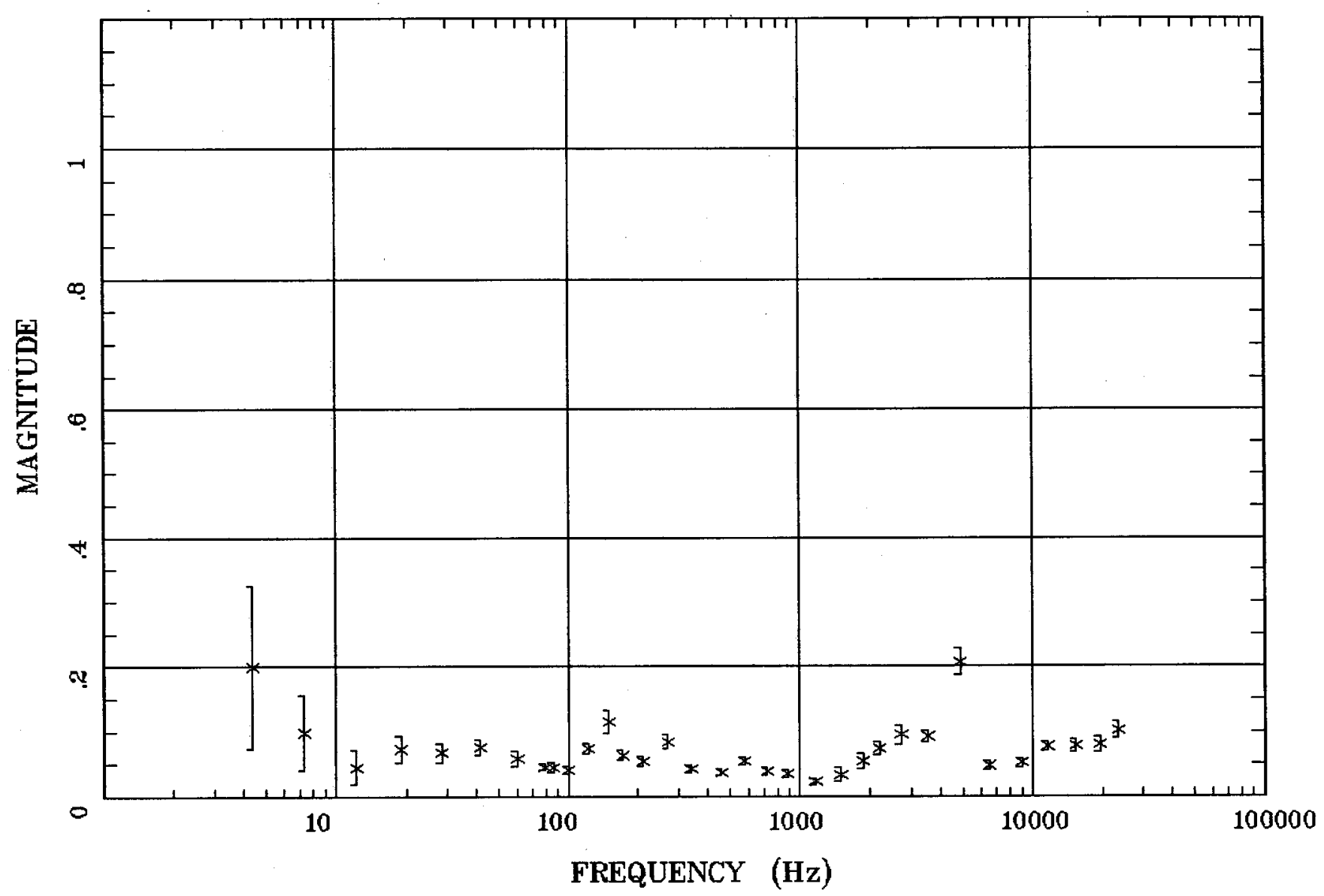

Client: Espanola Basin Remote: none

Acquired: 14:1 Jul 21, 2004 Survey Co:USGS
Rotation:

Filename: rr22a.avg

Channels: Ch1 Ch2 Ch3 Ch4 Ch5 Ch3 Ch4

Plotted: 11:37 Jan 12, 2006

< EMI - ElectroMagnetic Instruments 




Client: Espanola Basin Remote: none Acquired: 14:1 Jul 21, 2004 Survey Co:USGS
Rotation:

Filename: rr22a.avg

Channels: Ch1 Ch2 Ch3 Ch4 Ch5 Ch3 Ch4

Plotted: 11:38 Jan 12, 2006

< EMI - ElectroMagnetic Instruments 


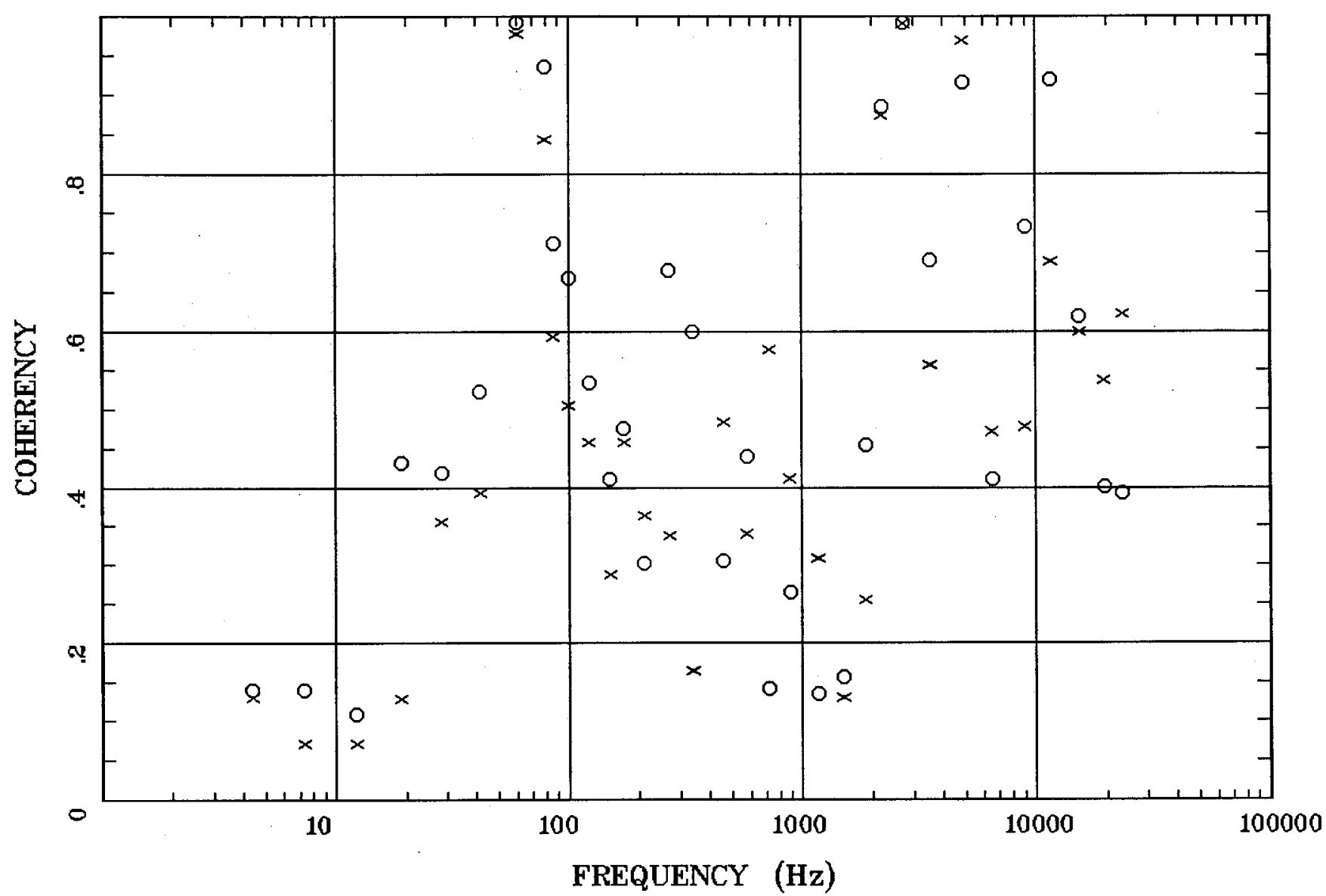

Client: Espanola Basin

Remote: none

Acquired: 14:1 Jul 21, 2004

Survey Co:USGS
Rotation:

Filename: rr22a.avg

Channels: Ch1 Ch2 Ch3 Ch4 Ch5 Ch3 Ch4

Plotted: 11:38 Jan 12, 2006

< EMI - ElectroMagnetic Instruments 


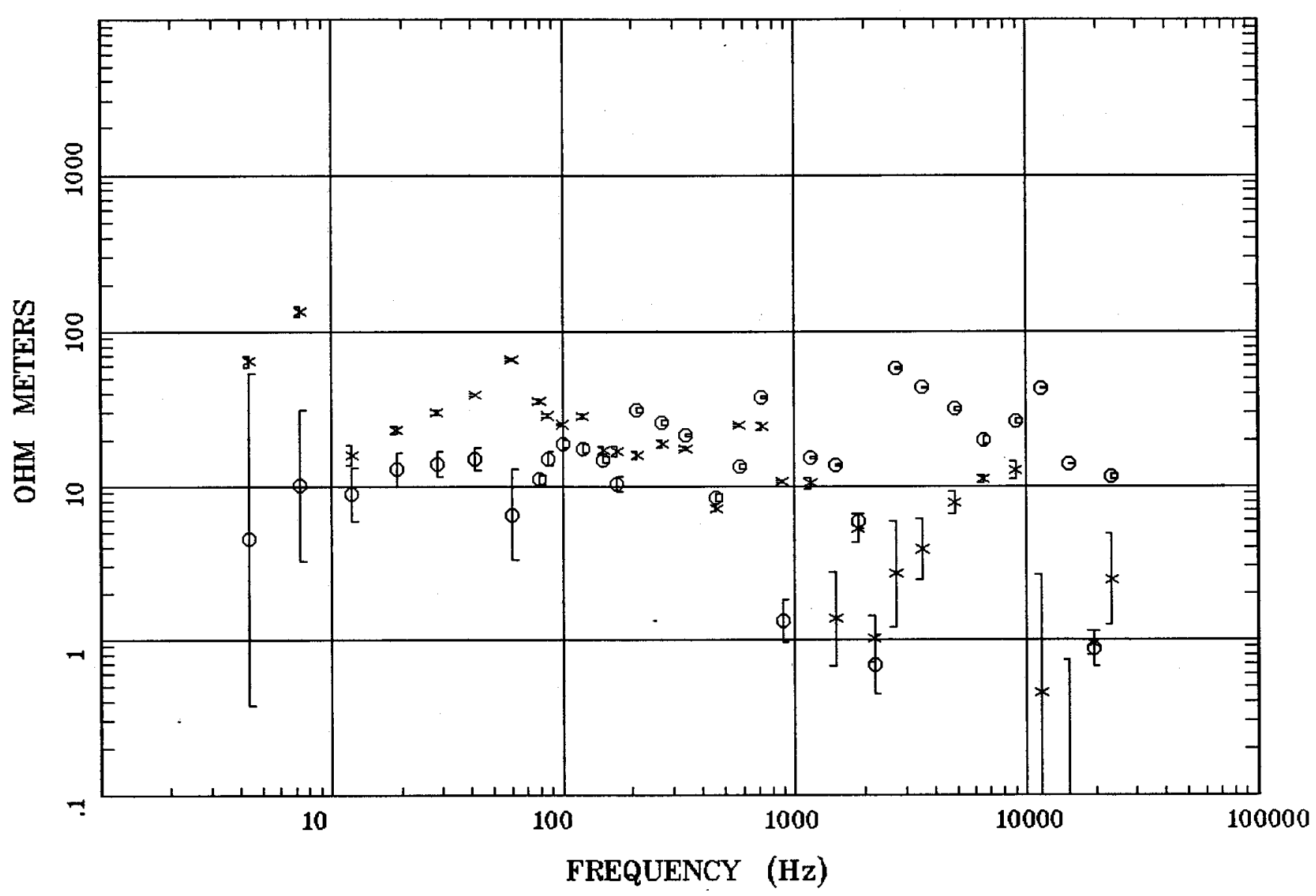

Client: Espanola Basin

Remote: none

Acquired: 13:4 Jul 19, 2004

Survey Co:USGS
Rotation:

Filename: rr23a.avg

Channels: Ch1 Ch2 Ch3 Ch4 Ch5 Ch3 Ch4

Plotted: 11:39 Jan 12, 2006

< EMI - ElectroMagnetic Instruments 


\section{IMPEDANCE PHASE}

Albuquerque $100 \mathrm{~K}$

Station RR23

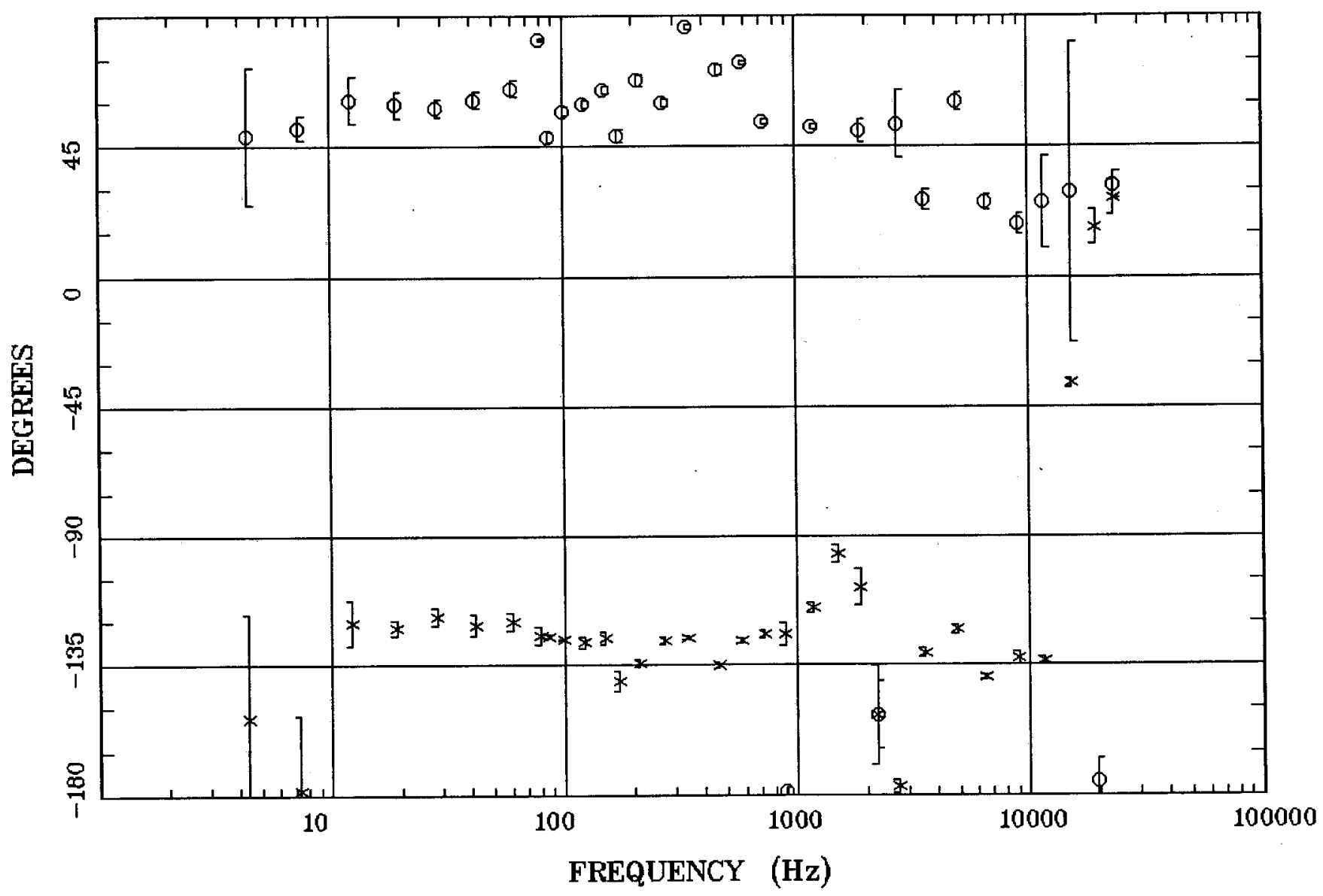

Client: Espanola Basin

Remote: none

Acquired: 13:4 Jul 19, 2004

Survey Co:USGS
Rotation:

Filename: rr23a.avg

Channels: Ch1 Ch2 Ch3 Ch4 Ch5 Ch3 Ch4

Platted: 11:39 Jan 12, 2006

< EMI - ElectroMagnetic Instruments > 


\section{ROTATION ANGLE}

Albuquerque $100 \mathrm{~K}$

Station RR23

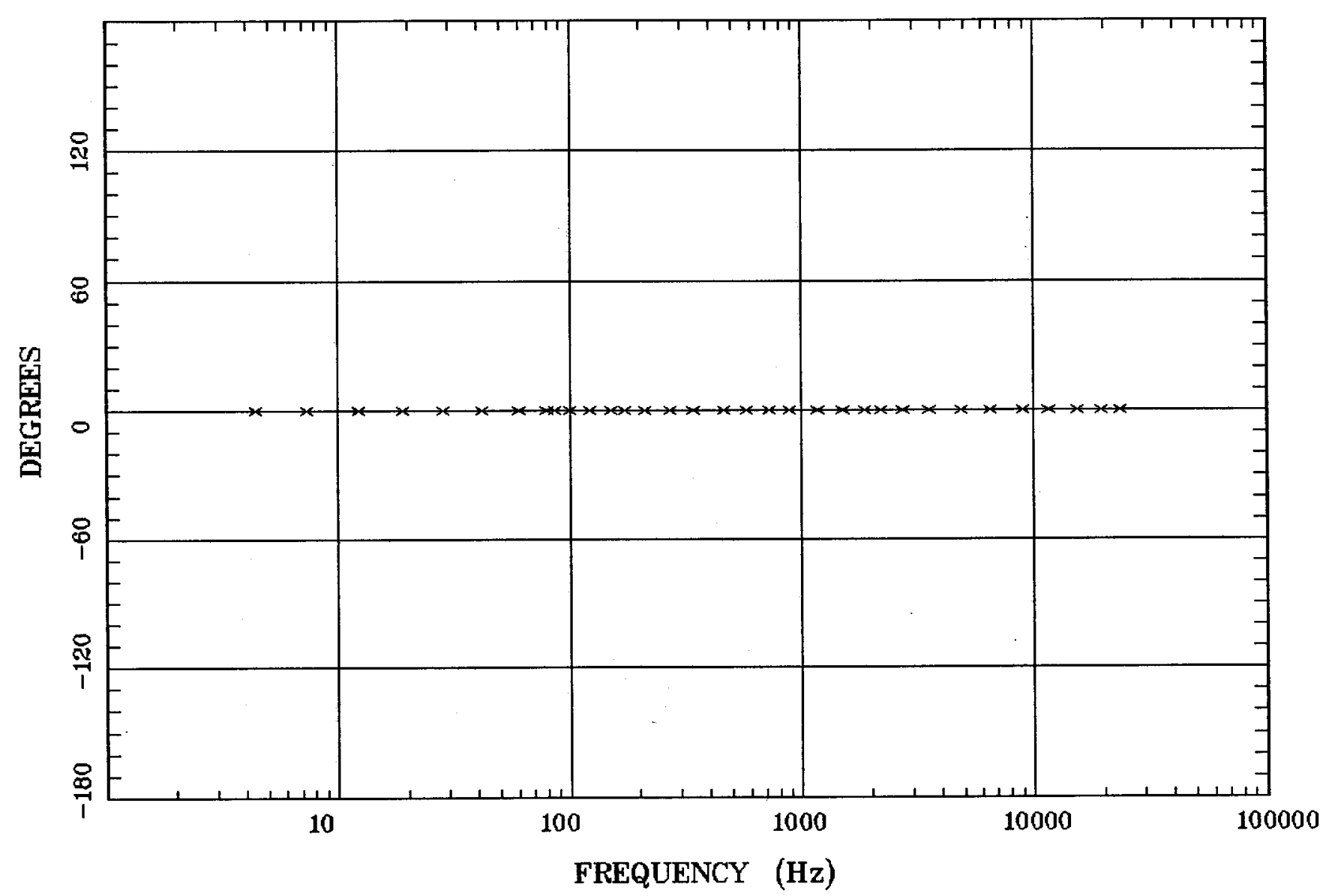

Client: Espanola Basin

Remote: none

Acquired: 13:4 Jul 19, 2004

Survey Co:USGS
Rotation:

Filename: rr23a.avg

Channels: Ch1 Ch2 Ch3 Ch4 Ch5 Ch3 Ch4

Plotted: 11:39 Jan 12, 2006

< EMI - ElectroMagnetic Instruments 


\section{IMPEDANCE SKEW}

Albuquerque $100 \mathrm{~K}$

Station RR23

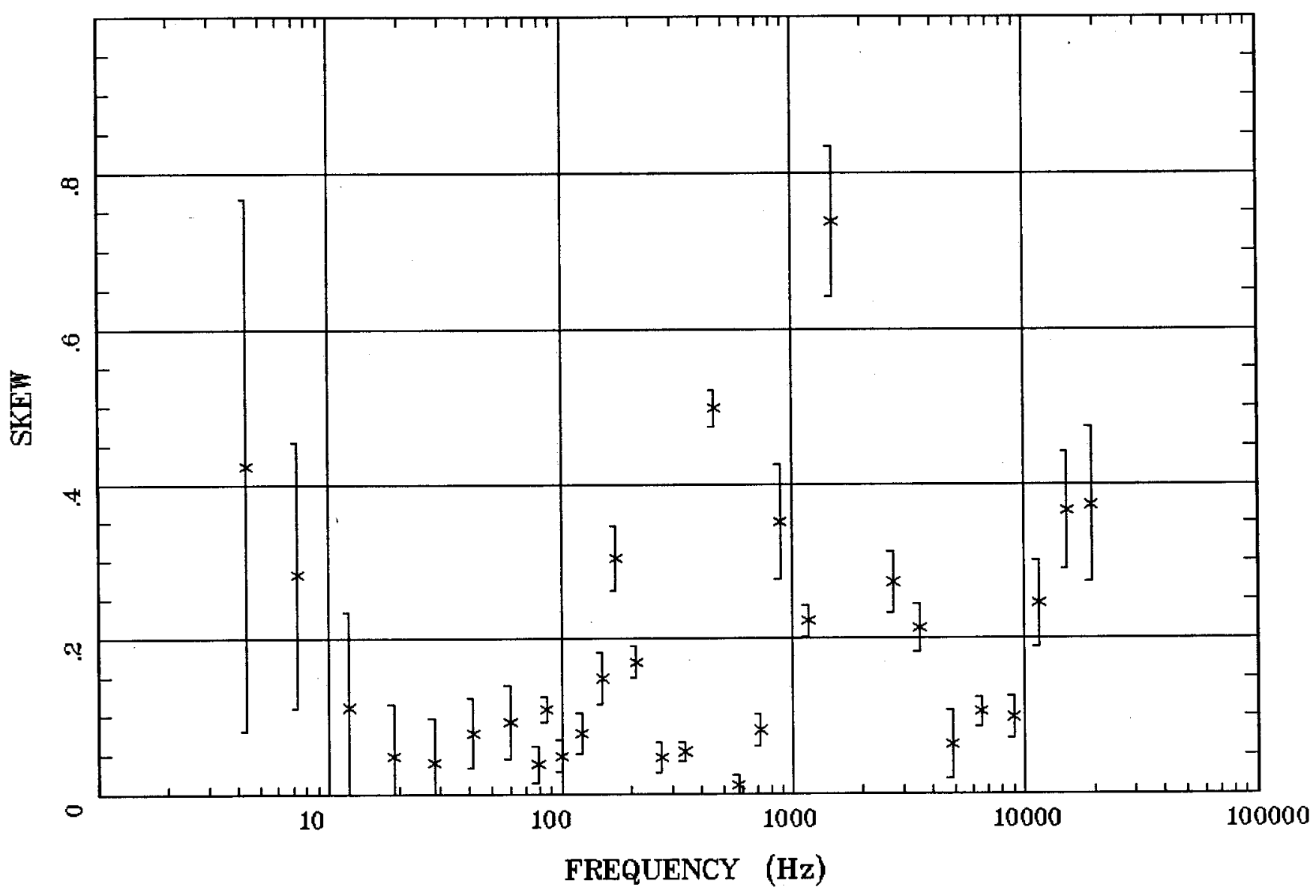

Client: Espanola Basin

Remote: none

Acquired: 13:4 Jul 19, 2004 Survey Co:USGS
Rotation:

Filename: rr23a.avg

Channels: Ch1 Ch2 Ch3 Ch4 Ch5 Ch3 Ch4

Plotted: 11:39 Jan 12, 2006

< EMI - ElectroMagnetic Instruments > 


\section{E MULT Coh.}

Albuquerque $100 \mathrm{~K}$

Station RR23

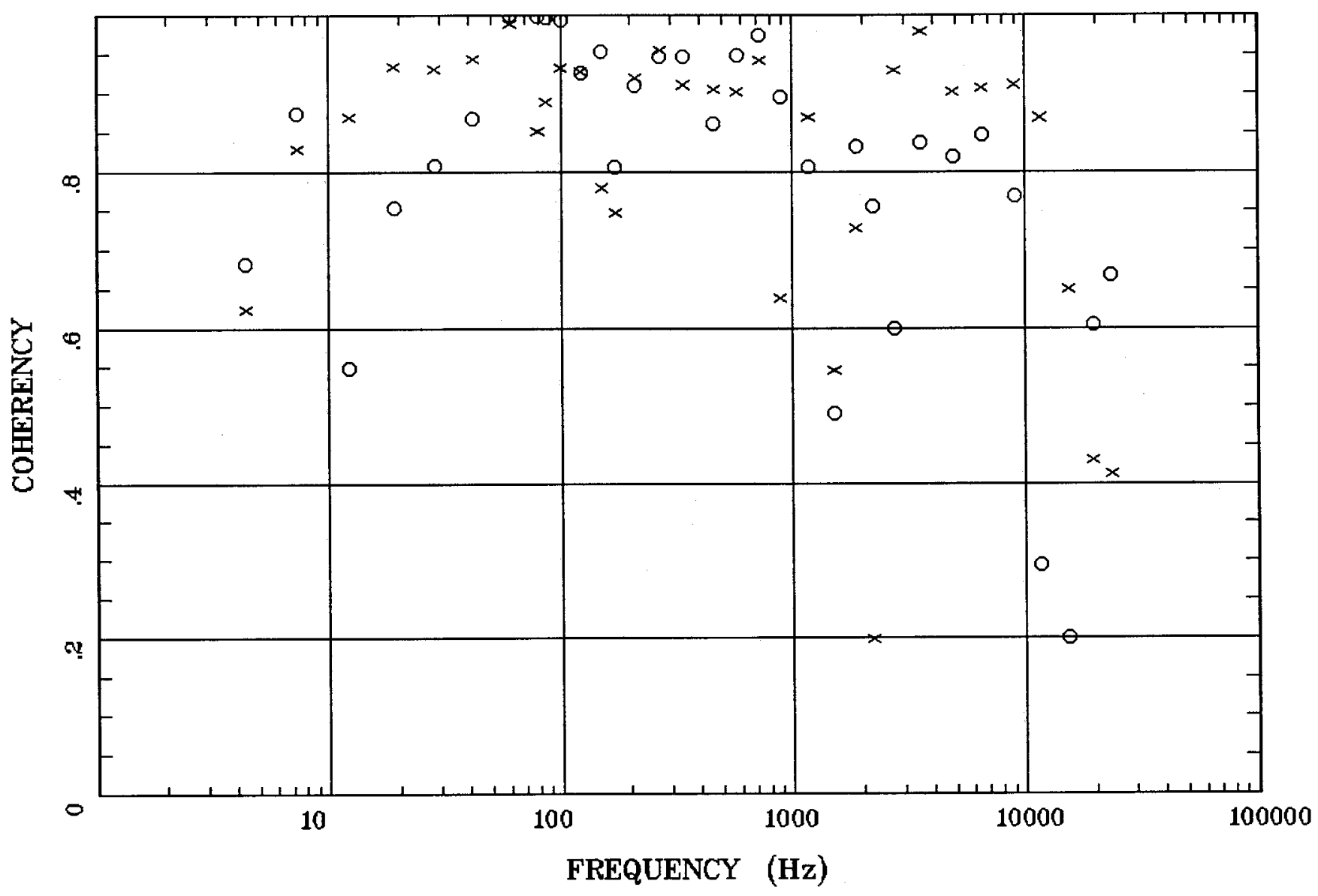

Client: Espanola Basin

Remote: none

Acquired: 13:4 Jul 19, 2004 Survey Co:USGS
Rotation:

Filename: rr23a.avg

Channels: Ch1 Ch2 Ch3 Ch4 Ch5 Ch3 Ch4

Plotted: 11:39 Jan 12, 2006

< EMI - ElectroMagnetic Instruments > 


\section{POLAR PLOTS}

Albuquerque $100 \mathrm{~K}$



Client: Espanola Besin

Remote: none

Acquired: 13:4 Jul 19, 2004

Survey Co:USGS
Rotation:

Filename: rr23a.avg

Channels: Ch1 Ch2 Ch3 Ch4 Ch5 Ch3 Ch4 Plotted: 11:39 Jan 12, 2006

< EMI - ElectroMagnetic Instruments > 


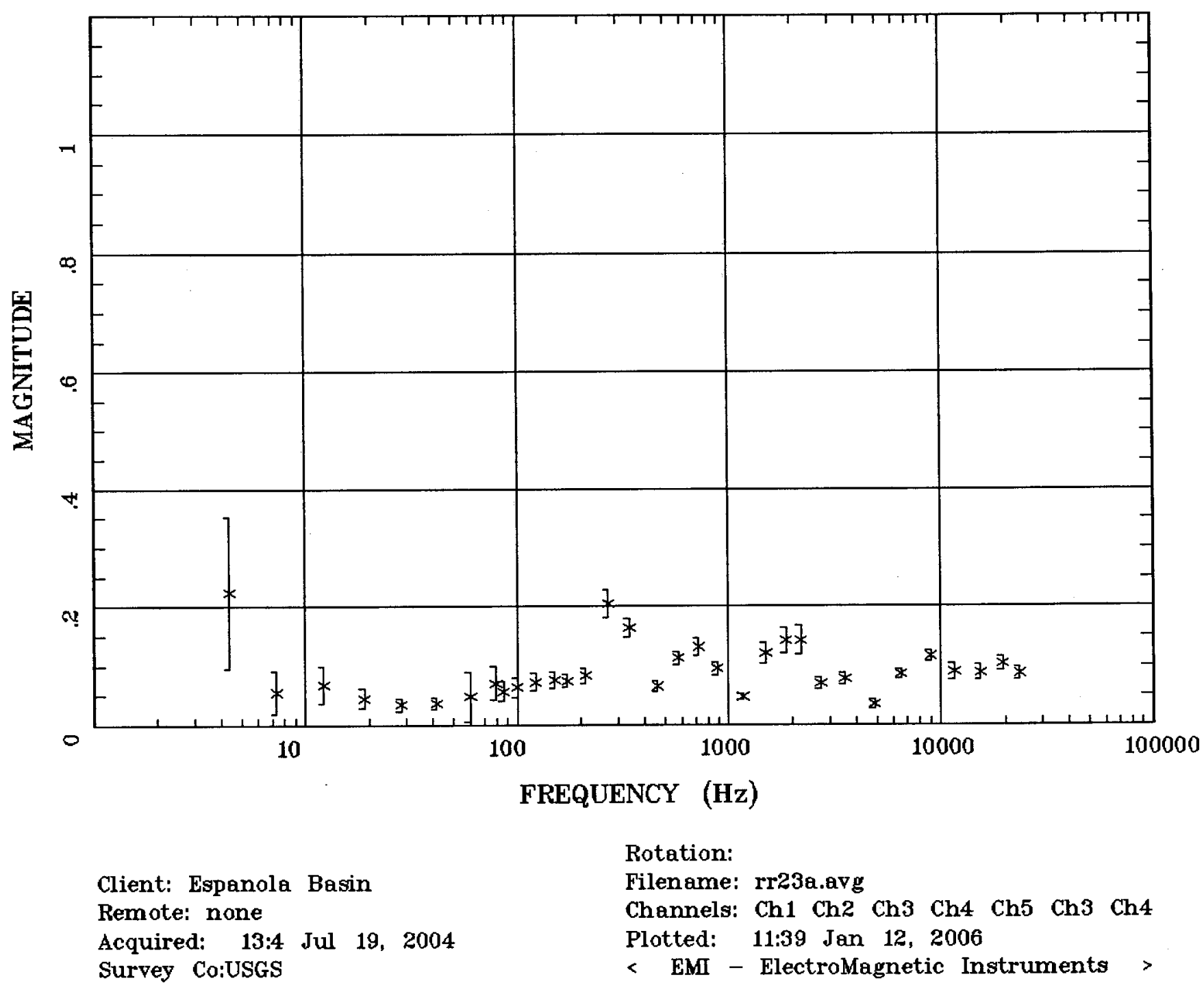




\section{TIPPER STRIKE}

Albuquerque $100 \mathrm{~K}$

Station RR23



Client: Espanola Basin

Remote: none

Acquired: 13:4 Jul 19, 2004 Survey Co:USGS
Rotation:

Filename: rr23a.avg

Channels: Ch1 Ch2 Ch3 Ch4 Ch5 Ch3 Ch4

Plotted: 11:39 Jan 12, 2006

< EMI - ElectroMagnetic Instruments > 


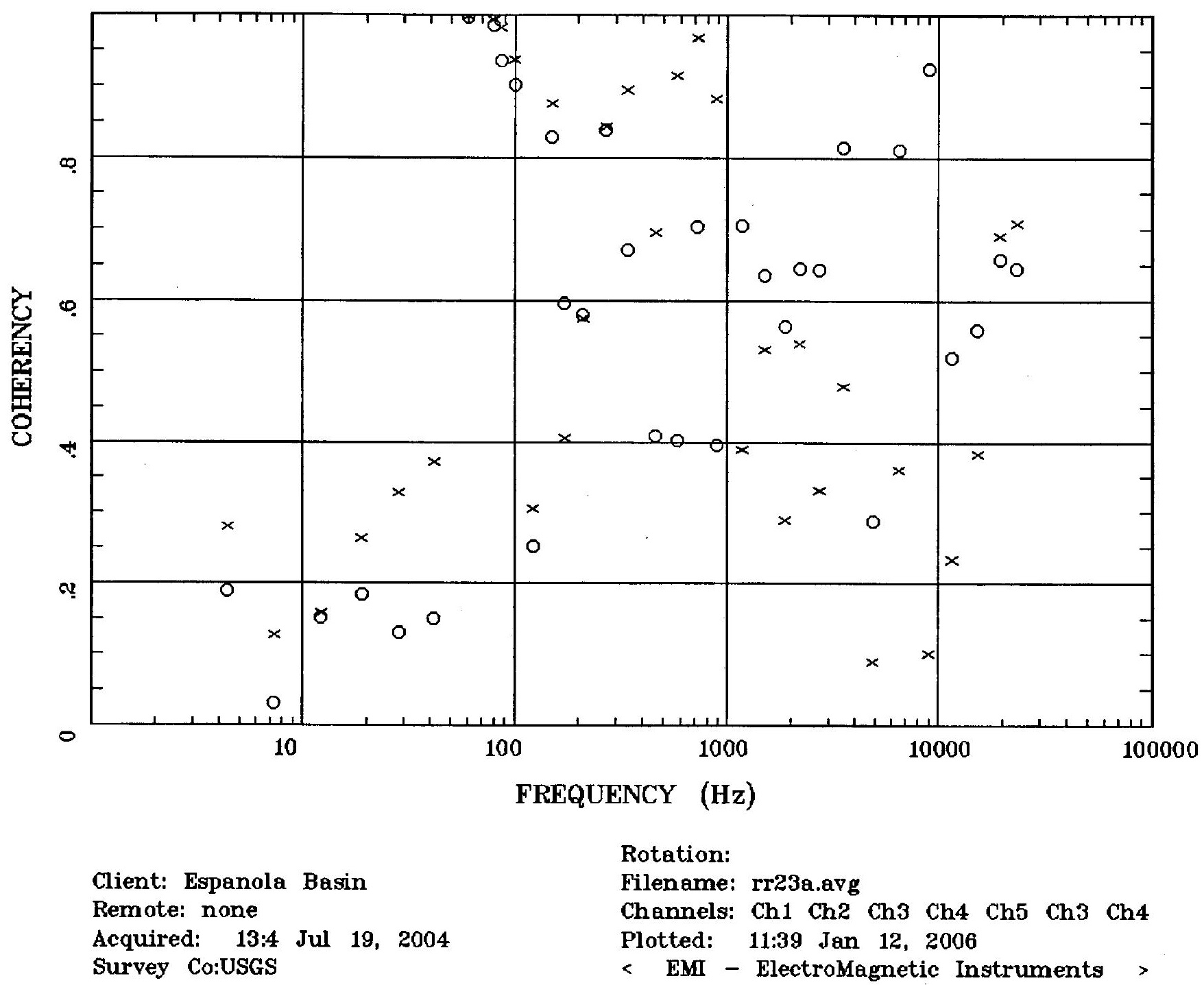

